Argonne

\title{
Optimization Framework for Solar Energy Integrated Resilient Distribution Grid
}

Energy Systems Division 


\begin{abstract}
About Argonne National Laboratory
Argonne is a U.S. Department of Energy laboratory managed by UChicago Argonne, LLC under contract DE-AC02-06CH11357. The Laboratory's main facility is outside Chicago, at 9700 South Cass Avenue, Lemont, Illinois 60439. For information about Argonne and its pioneering science and technology programs, see www.anl.gov.
\end{abstract}

\title{
DOCUMENT AVAILABILITY
}

Online Access: U.S. Department of Energy (DOE) reports produced after 1991 and a growing number of pre-1991 documents are available free at OSTI.GOV (http://www.osti.gov/),

a service of the US Dept. of Energy's Office of Scientific and Technical Information.

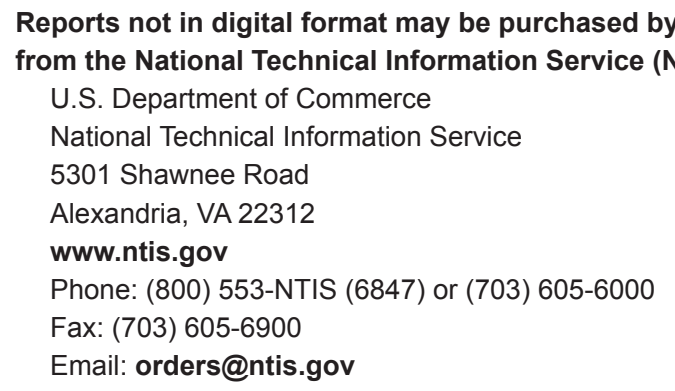

Reports not in digital format are available to DOE and DOE contractors from the Office of Scientific and Technical Information (OSTI):

U.S. Department of Energy

Office of Scientific and Technical Information

P.O. Box 62

Oak Ridge, TN 37831-0062

www.osti.gov

Phone: (865) 576-8401

Fax: (865) 576-5728

Email: reports@osti.gov

\begin{abstract}
Disclaimer
This report was prepared as an account of work sponsored by an agency of the United States Government. Neither the United States Government nor any agency thereof, nor UChicago Argonne, LLC, nor any of their employees or officers, makes any warranty, express or implied, or assumes any legal liability or responsibility for the accuracy, completeness, or usefulness of any information, apparatus, product, or process disclosed, or represents that its use would not infringe privately owned rights. Reference herein to any specific commercial product, process, or service by trade name, trademark, manufacturer, or otherwise, does not necessarily constitute or imply its endorsement, recommendation, or favoring by the United States Government or any agency thereof. The views and opinions of document authors expressed herein do not necessarily state or reflect those of the United States Government or any agency thereof, Argonne National Laboratory, or UChicago Argonne, LLC.
\end{abstract}


ANL-ESD-21/15

\section{Optimization Framework for Solar Energy Integrated Resilient Distribution Grid}

by

Bo Chen ${ }^{1}$, Shijia Zhao1, Chen Chen', Zhaoyu Wang², Arif Anmar², Shanshan Ma², Jiayong $\mathrm{Li}^{3}$, Mohammad Khodayar ${ }^{3}$

${ }^{1}$ Energy Systems, Argonne National Laboratory

${ }^{2}$ lowa State University

${ }^{3}$ Southern Methodist University

Acknowledgment: This material is based upon work supported by the U.S. Department of Energy's Office of Energy Efficiency and Renewable Energy(EERE) under the Solar Energy Technologies Office Award Number 34228.

December 2021 

Final Technical Report (FTR)

\begin{tabular}{|c|c|}
\hline a. Federal Agency & Department of Energy \\
\hline b. Award Number & CPS\# 34228 \\
\hline c. Project Title & $\begin{array}{l}\text { Optimization Framework for Solar Energy } \\
\text { Integrated Resilient Distribution Grid }\end{array}$ \\
\hline d. Principal Investigator & $\begin{array}{l}\text { Name: Bo Chen } \\
\text { Title: Energy Systems Scientist/Computational } \\
\text { Engineer } \\
\text { Email address: } \frac{\text { bo.chen@anl.gov }}{(630) 252-4464} \\
\text { Phone number: }\end{array}$ \\
\hline e. Business Contact & $\begin{array}{l}\text { Name: Sandra A. Davis } \\
\text { Title: Assistant Division Director } \\
\text { Email address: } \text { sdavis@anl.gov } \\
\text { Phone number: }(630) 252-8259\end{array}$ \\
\hline f. Submission Date & $05 / 15 / 2020$ \\
\hline g. DUNS Number & DUNS Number \\
\hline h. Recipient Organization & Argonne National Laboratory \\
\hline i. Project Period & Start: 10/01/2018 \\
\hline j. Submitting Official Signature & \\
\hline
\end{tabular}

\section{Acknowledgment:}

This material is based upon work supported by the U.S. Department of Energy's Office of Energy Efficiency and Renewable Energy (EERE) under the Solar Energy Technologies Office Award Number 34228.

Executive Summary:

The increasing penetration of distributed photovoltaic (PV) energy and other distributed energy resources (DERs) such as energy storage batteries, diesel generators, and mobile generators deployed in distribution grids must be properly controlled and coordinated to ensure reliable, resilient, and affordable grid operation in response to various operating conditions. Particularly, weather-induced power outages, such as natural disasters, are among the most common causes of power supply interruptions for distribution grid operations [E-1]. Distributed PV and DERs feature advanced grid supporting capabilities and can potentially enhance power supply continuity during and after extreme weather events. To leverage PV and other DERs to provide resilience benefits while ensuring operational and economic feasibility, distribution grids must be carefully designed, proactively managed, and safely operated through a comprehensive framework. 
This project aims to develop a multi-time scale optimization framework to facilitate the benefits of distributed solar energy in resilience improvement of distribution grid against disastrous events and ensure a 5-day islanded operation supported by DERs after the events causing extended power outage [E-2]. In the framework, solar energy is coordinated with other flexible resources to achieve optimal decisions ranging from preevent preparation to post-event operation. Uncertainties caused by various factors such as extreme weather forecast, solar irradiance, load demand, and damage prediction are integrated by utilizing advanced mathematical optimization techniques and solution algorithms.

In this project, the project team has successfully developed a stochastic optimization framework featuring advanced optimization models and solution algorithms to provide optimal solutions for planning and coordinating various resources (e.g., substations, mobile generators, diesel generators, repair crew, and materials), before and after the occurrence of natural disasters. Specifically, the stochastic optimization framework consists of three models: 1) Pre-event resource allocation model based on two-level stochastic optimization with progressive uncertainty hedging; 2) Post-event restoration model based on a first-of-a-kind routing-based model to coordinate restoration and crew dispatch, and 3) Energy management model with multi-scale coordination to ensure 5day continuous operation. The project team has demonstrated the effectiveness of the proposed framework on both large-scale test systems and real utility feeders with various PV penetration levels. The unserved energy can be reduced by $20 \%$ for a small-scale benchmark system and 30\% for a large-scale test system. The project resulted in 4 journal papers, 1 conference paper, and 7 conference presentations.

The developed framework enables a flexible operation paradigm toward improving the distribution grid resilience by fully leveraging the controllability, flexibility, and locational value of solar energy. Also, the framework can provide utilities with more cost-effective resource planning solutions before the extreme event and reduce PV and load curtailment during extreme conditions, and eventually enable solar energy to play a critical role in improving distribution grid resilience and further promote renewable energy deployment. Acronyms:

\begin{tabular}{|c|c|c|c|}
\hline BESS & Battery energy storage system & MES & Mobile energy storage \\
\hline Cl & Confidence interval & MILP & Mixed-integer linear programming \\
\hline CVar & Conditional value-at-risk & MRP & Multiple replication procedure \\
\hline DER & Distributed energy resource & OA & Operation agent \\
\hline DG & Distributed generator & OMS & Outage management system \\
\hline DSO & Distribution system operator & OPF & Optimal power flow \\
\hline DSR & Distribution system restoration & PH & Progressive hedging \\
\hline EA & Energization agent & PV & Photovoltaic \\
\hline EF & Extensive form & RA & Repair agent \\
\hline ESS & Energy storage system & RO & Robust optimization \\
\hline IAB & Industry Advisory Board & SMIP & Stochastic mixed-integer linear program \\
\hline MEG & Mobile energy generator & SP & Stochastic programming \\
\hline
\end{tabular}




\section{Background:}

Modern power systems are threatened by the increasing frequency and severity of extreme weather events, which sometimes can cause a catastrophic impact on energy infrastructure and extended outage duration (e.g., several days or even weeks). Among all the power outages, distribution networks contribute over $90 \%$ of customer outageminutes in the United States [E-1]. Since modern societies are utterly relying upon electricity, it is critical to improve grid resilience by harnessing the grid infrastructure and coordinating grid resources through the duration of extreme events. As required by the funding opportunity, one of the project goals is to ensure a 5-day operation in this kind of situation. Emerging DERs and various smart grid technologies deployed in modern distribution systems demonstrate great potential that can be leveraged for improving grid resilience. These resources can be potentially coordinated to form and operate as a temporary microgrid to maintain continuity of operation of critical loads in response to major weather events for an extended period.

The developed framework systematically integrate pre-event resource planning and postevent restoration and management, both of which are typical optimization problems focusing on the coordination of PV and other DERs (i.e., energy storage batteries, mobile diesel generators, and backup generators), controllable grid assets (i.e., switches, on-line tap changers, voltage regulators, and capacitor banks), and crews under a set of damage scenarios. Resource allocation, service restoration, and energy management problems have been extensively researched in the literature. However, new challenges are identified in the context of this project.

\section{Pre-event Preparation}

In the context of operational measures for preventive preparation in the resilience enhancement of electric distribution systems, many researchers have investigated preevent resource allocation problems. In [B-1], repair crew scheduling is integrated with the restoration process to enhance the resilience of electric distribution systems. In [B-2], the formulation is extended to a two-stage stochastic mixed-integer program model to solve the repair and restoration problem considering the stochastic nature of the repair time and the customer load demand. In [B-3], a two-stage stochastic mathematical model is developed to select staging locations and allocate crews and equipment for disaster preparation with considering constraints of system operation and routing problems.

However, there remain some limitations in the above studies on the pre-event preparation and resource allocation for an upcoming event. First, the coordination of multiple mobile resources, such as mobile generators and pre-staging repair crews, is not comprehensive. Second, the weather-induced uncertainties are simply generated by the grid component fragility model which only represents the general probability that a grid component will reach a design limit state within a given period. It cannot model the temporal correlation of damages or provide the exact damaged time. Moreover, the assumption of how photovoltaic (PV) systems respond during outages does not represent the full capability of PV. 
In this project, we propose a two-stage stochastic mixed-integer linear program, where the first stage allocates mobile emergency generators, battery energy storage system (BESS), fuel, repair crews, and the second stage considers constraints of unbalanced power operation, reconfiguration operation, and repair schedule. Advanced weather forecasting methodologies and fragility models [B-4]-[B-5] are applied here to generate damage scenarios with full consideration of the damage to the system and the affected areas. A framework for integrating different types of PV systems in the restoration process is also developed to further enhance the resilience of the system and decrease the restoration time.

\section{Post-event Restoration}

The current practice adopted by utilities for managing natural disasters normally manages the service restoration and crew dispatch by two different groups of operators, since they are executed at different time scales and involve different expertise [B-6]. In face of natural disasters, there is limited coordination between the restoration group and the crew dispatch group, resulting in inefficient restoration performance. In the literature, cooptimization of restoration and crew dispatch has been proposed in a few papers to achieve better resilience improvement. Nevertheless, the research on DSR and crew dispatch co-optimization is still at its early stage with many concerns unaddressed. In [B8], a mixed-integer linear programming (MILP) model is introduced to assign repair crews to damaged components while considering the restoration preference but ignoring the travel time and routing sequence. The authors in [B-9][B-10][B-11] decoupled power restoration and crew routing problems to improve the computation efficiency at the cost of compromising the solution optimality. Our ISU team has formulated the problem using a fix-time step model and applied heuristic techniques [B-1][B-2]. The results demonstrated the advantages of integrating DSR and crew repair problems over traditional methods. However, there are still several challenges to be addressed in the context of this project: 1) the computational time for large-scale test systems. Because the number of decision and state variables will increase as the size of the test system grows, the computational time required to solve the optimization problem will increase exponentially. 2) The optimality of the solutions. The integrated model using formal optimization models, on the other hand, can be solved by off-the-shelf solvers and track the optimality gap during the problem-solving process. 3) The interdependency between restoration and crew dispatch logics.

To address the abovementioned challenges, the project team has developed a first-of-akind post-event restoration model by adapting the vehicle routing model and existing power system models including three-phase unbalanced power flow and operational constraints (e.g., voltage and line capacity constraints, DER capacity, and generation constraints).

\section{Energy Management of DERs and Microgrids}

Post-event energy management in the distribution network can be regarded as a variation of the optimal power flow problem with different objectives which has been extensively 
investigated in previous publications. To name a few, the energy management formulation proposed in [B-12] minimizes the network loss and improve the load balancing, the objective in [B-14] is to minimize the operation cost and network lossand the objective in ref $[B-15]$ is to minimize the load shedding. Other objectives including improving system reliability [B-16]-[B-18] and improving voltage profile [B-18], were addressed in the literature. Various types of operation constraints have been captured in the existing research works, including the real and reactive power generation of distributed generation resources [B-14], the tap of the voltage regulators [B-19], capacitor banks [B-20], and demand response constraints [B-21]. However, these works cannot adapt to the post-event operation as they fail to incorporate the post-event operation constraints, such as switch action sequence, availability of distribution branches, grid forming, and grid following features of DERs, as well as the real-time implementations. The distribution networks (DNs) are fundamentally unbalanced due to variation of load on phases and the untransposed distribution branches. The energy management problem for the unbalanced DNs is generally formulated as a nonconvex optimization problem. To solve this problem, interior point method [B-22], quasi-newton method [B-23], linearization [B-12], and convex relaxation techniques [B-24]-[B-29] are derived for the non-convex constraints with high accuracy. Second-order cone programming relaxation was used to solve this problem in [B-30]. The energy management problem is formulated as mixed integer nonlinear programming problem and heuristic and meta-heuristic approaches, including Fuzzy logic [B-31]-[B-33], Particle Swarm Optimization [B-34], Genetic Algorithm [B-35], Ant Colony [B-36], and Tabu search [B-37] algorithms were used to solve the energy management problem. Finally, multi-agent systems were proposed to solve energy management problems in distribution network [B-38], [B-39], and to achieve faster solution, distributed algorithms are proposed in [B-29], [B-40].

Ref. [B-41] proposes a linearized multi-phase DistFlow model which is proven to be a good approximation of the original nonconvex DistFlow model. Thus, it is adopted in this project to account for the coupling effect between different phases.

Furthermore, a rolling horizon approach that is employed for real-time energy management in distribution network [B-42], [B-43] and similar approach is adopted in this project. Different from the most recent works the proposed research also incorporates the switching action and restoration sequences and hence can well adapt to the post-event restoration sequence. To handle the long-duration operations, such as 5-day operation, a two time-scale operation strategy is developed, where the large time-scale operation considers a larger operation horizon (i.e. 5 days) that provides a reference point for the small time-scale operation which incorporates the up-to-date system information. Consequently, a tradeoff between reducing computation time and avoiding shortsightedness is achieved.

In order to address the uncertainties in the power system operation, stochastic programming $(\mathrm{SP})$ and robust optimization $(\mathrm{RO})$ are widely used in the literature $[\mathrm{B}$ $44],[B-45]$. In [B-45], a robust restoration model was presented where the DER outputs 
and demands are represented by an adjustable uncertainty set. Nevertheless, the robust optimization considers the worst-case scenario which rarely happens in practice. Hence, it would result in over-conservativeness. In [B-46], the uncertainties in loads and renewable generations are captured by the multiple uncertainty scenarios generated from the predefined probability distribution functions using the Monte Carlo method. This project leverages the same technique to characterize the uncertainties in solar irradiance.

\section{PV Types and Placement}

To investigate the benefits of PV at different penetration levels, we have developed a set of test scenarios by allocating different numbers and different types of PVs in the system. The general assumptions and definitions of PV types are summarized in Table B-1 and referred to in the following sections of this report to avoid duplicative descriptions. The storage size (i.e., 8 hours) is selected based on the maximum duration of supply suggested by recent reports and products on the market [B-47],[B-48],[B-49], as well as the predictions on the size of Li-lon batteries by 2030 (i.e., 6 - 8 hours) [B-50].

\begin{tabular}{|c|c|c|c|c|}
\multicolumn{5}{|c|}{ Table B-1. Configuration of Three Types of PV } \\
\hline Type & Model & Storage & Mode & Dispatch \\
\hline III & Large utility PV farm & 8 hours of supply & $\begin{array}{c}\text { Grid-forming } \\
\text { Grid-following }\end{array}$ & Dispatch-able \\
\hline II & Midsize PV system & 8 hours of supply & Grid-following & Dispatch-able \\
\hline I & Residential PV panel & N.A & Grid-following & MPPT \\
\hline
\end{tabular}

As hosting capacity analysis is beyond the scope of the project, a simplified heuristic rule is adopted by the team for PV placement. Large utility PV farms or Type III, are placed at the feeder backbone nodes. Type I and Type II PVs, are both placed at "leaf" nodes. However, Type I PVs would have fewer nodes connected to them compared with Type II PVs. Based on these criteria, each specific PV in corresponding types is randomly placed on buses meeting the criteria. Different from Type I and Type II, Type III is assumed to have grid forming capability.

\section{Resilience Metrics}

In this project, two resilience metrics are defined to evaluate the resilience improvement [B-51]: 1) Cumulative customer energy demand not served, and 2) time to recovery.

Cumulative customer energy demand not served is defined as how much the cumulative energy not served through the outage duration for all customers, and it is measured in $\mathrm{kWh}$. Time to recovery, or outage duration, is defined as the time duration to fully restore all the customers, and it is measured in hours.

\section{Project Objectives:}

\section{Impact:}

The developed framework enables a flexible operation paradigm toward improving the distribution grid resilience by fully leveraging the controllability, flexibility, and locational value of solar energy. In addition, the framework can provide customers with more costeffective resource planning solutions before the extreme event and reduce PV and load 
curtailment during extreme conditions. Eventually, it can enable solar energy to play a critical role in improving distribution grid resilience and further promote renewable energy deployment.

\section{Project Objectives:}

The overall objective is to develop optimization models and solution algorithms to facilitate the benefits of distributed solar energy in the resilience improvement of distribution grids. The proposed work will incorporate the following objectives:

1. Develop a pre-event preparation optimization model and solution algorithms such that flexible resources and distributed solar energy can be prepared strategically for the upcoming extreme event

2. Develop post-event operation and restoration optimization models and solution algorithms to realize the benefits of solar energy in grid resilience

3. Evaluate the developed framework on both a real-world test system and a largescale test system with more than 10,000 nodes

\section{Expected Outcomes:}

1. Develop the optimization models and solution algorithms for pre-event proactive management and post-event operation.

a. The model should be tested on small-scale test systems, large-scale test systems (>10,000 nodes), and a real feeder system.

b. For the pre-event proactive management, the computation time for both large-scale test systems and real feeder systems should be within the expected threshold (e.g., 4 hours). Similar to the post-event operation (5 min for energy management optimization and 1 hour for restoration optimization).

c. The resilience improvement should be at least $10 \%$ in terms of served energy and reduction of outage duration as compared to benchmark results based on existing utility practices for resource allocation and restoration.

2. A comprehensive technical report that describes the optimization models, solution algorithms, and case studies via simulations.

3. Quarterly reports

4. Two peer-reviewed journal papers

\section{Significance, Innovation, and Fundamental Advances:}

The contribution of this project is two-fold: 1) providing optimal and coordinated decisionmaking tools for both pre-event preparation and post-event operation of solar energy in coordination with flexible resources to achieve 5-day isolated operation and improve grid resiliency against extreme weather events; 2) providing a framework to quantify the resilience benefits of solar energy under emergency conditions. The proposed framework allows the system operators to flexibly coordinate different types of PV systems with 
respective controllability, and leverage the locational value of PV systems to facilitate grid operation. The resilience benefit evaluated by the proposed framework will help to achieve informed decision-making for PV deployment to achieve the desired resilience improvement target.

The methodologies developed in this project can be potentially adopted by various stakeholders. For example, electric utilities can use the work to guide the preparation and operation of flexible resources and distributed solar energy to improve supply continuity in extreme weather events. Regulatory agencies can utilize the work as a quantitative method to evaluate the resilience enhancement of the grid due to the participation of solar energy. Software vendors can use the work to improve outage management systems. Device vendors can use the work to specify functional requirements for devices to respond properly in extreme weather events.

\section{Tasks and Milestones}

Task

Task 1

Task 2

Set up an industrial advisory board (IAB) and deliver webinars

\section{Description}

Development of pre-event proactive management optimization models and solution algorithms.

M 1.2.1: Development of preliminary optimization models of the pre-event preparation and post-event operation completed; the optimization models and test cases setup mechanism are presented to the IAB

M 1.2.2: Development of solution algorithms for the refined optimization models with preliminary testing results via simulation on small-scale test cases generated from subtask 4.1 and reviewed by the IAB and the DOE team; the resilience improvement should be at $10 \%$ in terms of served energy and reduction of outage duration.

M 1.2.3: Development of solution algorithms for the refined optimization models with large-scale test cases developed in subtask 4.2; the resilience improvement should be at least $10 \%$ in terms of served energy and reduction of outage duration

\begin{tabular}{l|l} 
Task 3 & Development of Post-event operation and restoration optimization models and solution
\end{tabular} algorithms

M 1.3.1: Development of refined optimal energy management optimization model and solution algorithms with intermediate testing results based on small-scale test cases; the resilience improvement in terms of served energy will be at least $10 \%$.

M 1.3.2: Development of optimal restoration and load pick-up optimization model and solution algorithms using DERs, network reconfiguration, and repair crew and intermediate testing results based on small-scale test cases the resilience improvement in terms of reduction of outage duration will be at least $10 \%$.

M 1.3.3: Case studies of the solution algorithms of energy management optimization and restoration optimization under large-scale test cases with three-phase multiple feeders with at least 10,000 nodes generated within required computation time (5 min for energy management optimization and 1 hour for restoration optimization) completed; the resilience improvement will be at least $10 \%$ in terms of served energy and outage duration reduction.

Task 4 Setting up test cases used for pre-event preparation and post-event operation optimization solution algorithms

Go/No-Go Successful completion of milestones 1.2.3 and 1.3.3 with their corresponding metrics Milestone mentioned 


\begin{tabular}{|c|c|c|c|}
\hline \multicolumn{2}{|c|}{ Task 5} & \multicolumn{2}{|c|}{$\begin{array}{l}\text { Extensive case studies to evaluate the benefits of solar energy in resilience improvement } \\
\text { M 2.5.1: Case studies on the evaluation of benefits of solar energy and its coordination } \\
\text { with other flexible resources in grid resilience improvement; the impact of coordination } \\
\text { between pre-event and post-event optimization }\end{array}$} \\
\hline \multicolumn{2}{|c|}{ Task 6} & \multicolumn{2}{|c|}{$\begin{array}{l}\text { Testing of the pre-event and post-event optimization via simulation using real feeder data } \\
\text { M 2.6.1: Data interface development in software platforms (e.g., Matlab or Python) for the } \\
\text { real feeder data provided by utility partners. } \\
\text { M 2.6.2: Case studies of pre-event preparation optimization under real feeder data within } \\
\text { required computation time (e.g., } 4 \text { hours) completed and results being reviewed by the } \\
\text { utility; the resilience improvement will be at least } 10 \% \text { in terms of served energy and } \\
\text { outage duration reduction } \\
\text { M 2.6.3: Case studies of post-event operation optimization under real feeder data within } \\
\text { required computation time ( } 5 \text { min for energy management optimization and } 1 \text { hour for } \\
\text { restoration optimization) completed and results being reviewed by the utility; the resilience } \\
\text { improvement will be at least } 10 \% \text { in terms of served energy and outage duration reduction }\end{array}$} \\
\hline \multicolumn{4}{|c|}{$\begin{array}{l}\text { Project Results and Discussion: } \\
\text { High-level comparison of anticipated outcomes and realized results }\end{array}$} \\
\hline \multicolumn{3}{|c|}{ Anticipated Outcomes } & Realized Results \\
\hline \multicolumn{3}{|c|}{$\begin{array}{l}\text { The optimization models and solution algorithms } \\
\text { for pre-event proactive management and post- } \\
\text { event operation. } \\
\text { The model should be tested on a small-scale test } \\
\text { system, a large-scale test system ( }>10,000 \\
\text { nodes), and a real feeder system. } \\
\text { For the pre-event proactive management, the } \\
\text { computation time for both a large-scale test } \\
\text { system and a real feeder system should be within } \\
\text { the expected threshold (e.g., } 4 \text { hours). Similar to } \\
\text { the post-event operation ( } 5 \text { min for energy } \\
\text { management optimization and } 1 \text { hour for } \\
\text { restoration optimization). } \\
\text { The resilience improvement should be at } 10 \% \text { in } \\
\text { terms of served energy and reduction of outage } \\
\text { duration as compared to the benchmark. }\end{array}$} & $\begin{array}{l}\text { Completed development of optimization model } \\
\text { and algorithms } \\
\text { Model is tested on a small-scale test system, } \\
\text { large-scale test system (with } 14,319 \text { nodes), and } \\
\text { real feeder system with extensive case studies } \\
\text { Take the real feeder system as a demonstration } \\
\text { example, the computation time for pre-event } \\
\text { preparation is } 2.5 \text { hours, less than } 4 \text { hours } \\
\text { requirement, the post-event operation is } 7 \\
\text { minutes, which meets the project requirement. } \\
\text { The resilience improvement in both served load } \\
\text { energy improvement and outage duration } \\
\text { induction is higher than } 10 \% \text { compared with base } \\
\text { cases. }\end{array}$ \\
\hline \multicolumn{3}{|c|}{$\begin{array}{l}\text { A comprehensive technical report and quarterly } \\
\text { reports that describe the optimization models, } \\
\text { solution algorithms, and case studies via } \\
\text { simulations. }\end{array}$} & $\begin{array}{l}\text { A comprehensive report is delivered including } \\
\text { details on optimization model, algorithm, case } \\
\text { studies, as well as IAB feedbacks and comments. } \\
\text { Quarterly reports are submitted every quarter. }\end{array}$ \\
\hline \multicolumn{3}{|c|}{ Two peer-reviewed journal papers } & \\
\hline \multicolumn{4}{|c|}{$\begin{array}{l}\text { Project Tasks, Go/No-Go Milestones, and Deliverables } \\
\text { Task } 1 \text { Set up an industrial advisory board (IAB) and deliver webinars }\end{array}$} \\
\hline \multicolumn{4}{|c|}{ Description } \\
\hline T1 & \multicolumn{3}{|c|}{$\begin{array}{l}\text { Task Name: Set up an industrial advisory board (IAB) and deliver webinars } \\
\text { Task Description: Set up an IAB for the project and schedule webinars with IAB members to } \\
\text { receive their feedback on the project development. }\end{array}$} \\
\hline T1.1 & \multicolumn{3}{|c|}{ Set up IAB } \\
\hline
\end{tabular}




\begin{tabular}{|l|l|}
\hline & $\begin{array}{l}\text { Completion in Q1-FY19: Formed IAB including seven companies from utilities, vendor, and } \\
\text { research institute. }\end{array}$ \\
\hline T1.2 & $\begin{array}{l}\text { Webinar for the pre-event preparation and post-event energy management optimization } \\
\text { Completion in Q3-FY19: Delivered 2 webinars on 01/03/2019 and 7/12/2019 }\end{array}$ \\
\hline T1.3 & $\begin{array}{l}\text { Webinar for post-event restoration optimization model development, and intermediate results of } \\
\text { pre-event preparation and post-event energy management optimization } \\
\text { Completion in Q3-FY19: An additional webinar is delivered on 04/08/2020 to present the } \\
\text { developed framework, which received positive feedback from IAB members. }\end{array}$ \\
\hline $\begin{array}{l}\text { Deliverables: Delivered 3 webinars with IAB members on 01/03/2019, 7/12/2019 and 04/08/2020 } \\
\text { Report of detailed comments from IAB and corresponding responses }\end{array}$ \\
\hline
\end{tabular}

The project team invited experts from the industry and formed IAB with seven organizations including utilities, a vendor, and a research institute.

1. Tomas Tinoco Rubira, Aidan Tuohy - Electric Power Research Institute (EPRI);

2. Yoav Sharon - S\&C Electric Company;

3. Bill Muston - Oncor Electric Delivery;

4. Jeremy Richert - Maquoketa Valley Electric Cooperative;

5. Joe McGovern - Aliant Energy;

6. John Bilsten - Algona Municipal Utilities;

7. Chris Ball - City of Bloomfield Utility

The project team delivered three webinars during IAB member meetings on 01/03/2019, $7 / 12 / 2019$, and $04 / 08 / 2020$ to report the model development and progress. IAB members gave positive feedback on the importance of this project and provided several detailed comments regarding the application and path forward of this project. The project team provided corresponding responses. Key discussions are summarized in Table 1-1.

Table 1-1. Response to the Comments Received at IAB Meeting

\begin{tabular}{|l|l|}
\hline \multicolumn{1}{|c|}{ Comments } & \multicolumn{1}{c|}{ Response } \\
\hline $\begin{array}{l}\text { Official definition and categorization for } \\
\text { different types of PVs, and their differences }\end{array}$ & $\begin{array}{l}\text { IEEE1547 provides the categories of different PV types } \\
\text { according to their controllability, and Type I, II, and III } \\
\text { PVs are defined based on academic reference. }\end{array}$ \\
\hline $\begin{array}{l}\text { Differences between pre-event crew } \\
\text { dispatch and post-event crew dispatch }\end{array}$ & $\begin{array}{l}\text { Pre-event dispatch will assign crew to depots. Post-event } \\
\text { dispatch will determine the repair sequence. }\end{array}$ \\
\hline $\begin{array}{l}\text { Difference between stage II pre-event } \\
\text { model and the post-event model }\end{array}$ & $\begin{array}{l}\text { The project team illustrated that the differences are in the } \\
\text { level of operation details and level of uncertainties }\end{array}$ \\
\hline $\begin{array}{l}\text { Clarification on stage I and stage II in pre- } \\
\text { event stochastic optimization }\end{array}$ & $\begin{array}{l}\text { The project team introduced the stochastic optimization } \\
\text { model and the solution approach }\end{array}$ \\
\hline $\begin{array}{l}\text { Clarification on the test system setup } \\
\text { parameters: }\end{array}$ & $\begin{array}{l}\text { The project team provided illustrations on the PV levels } \\
\text { Definition of PV penetration, DER capacity } \\
\text { and grid operation mode, the definition of } \\
\text { resilience, generation of weather-induced } \\
\text { outages }\end{array}$ \\
$\begin{array}{l}\text { future presentation to better show the PV impact. The } \\
\text { definition of resilience and weather-induced outages are } \\
\text { clarified. }\end{array}$ \\
\hline
\end{tabular}




\begin{tabular}{l|l|}
$\begin{array}{l}\text { Clarification on the test system results: } \\
\text { resource allocation patterns; parameters in } \\
\text { resilience improvement; resilience } \\
\text { changing pattern according to penetration }\end{array}$ & $\begin{array}{l}\text { The project team provided a detailed illustration of results } \\
\text { analysis such as the load profile used, the pre- } \\
\text { determined critical / non-critical loads, and corresponding } \\
\text { devel increase; comparison between the } \\
\text { critical and non-critical load }\end{array}$ \\
$\begin{array}{l}\text { Suggestions on the presentation of the } \\
\text { the benefit of PV in restoration is not directly proportional } \\
\text { to PV penetration levels and provided a "turning point" } \\
\text { scenario analysis. }\end{array}$ \\
$\begin{array}{l}\text { The project team will update future presentations } \\
\text { accordingly. }\end{array}$ \\
\hline
\end{tabular}

Task 2 Development of pre-event proactive management optimization models and solution algorithms

\begin{tabular}{|c|c|}
\hline & Description \\
\hline T2 & $\begin{array}{l}\text { Task Name: Development of pre-event proactive management optimization models and } \\
\text { solution algorithms. } \\
\text { Task Description: Develop optimization models that prepare solar energy participation and } \\
\text { other flexible resources strategically before the event, such that these flexible resources can be } \\
\text { utilized optimally after the event. }\end{array}$ \\
\hline T2.1 & $\begin{array}{l}\text { State-of-the-art review of the pre-event management methodologies } \\
\text { Completion in Q1-FY19: Completed comprehensive review }\end{array}$ \\
\hline T2.2 & $\begin{array}{l}\text { Development of optimization model for pre-event preparation } \\
\text { Completion in Q2-FY19: A two-stage stochastic mixed-integer linear program has been } \\
\text { developed, where the first stage allocates mobile emergency generators, BESS, fuel, repair } \\
\text { crews, and the second stage includes constraints of unbalanced power operation, } \\
\text { reconfiguration operation, and repair scheduling }\end{array}$ \\
\hline $\begin{array}{l} \\
1.2 .1\end{array}$ & $\begin{array}{l}\text { Development of preliminary optimization models of the pre-event preparation and post-event } \\
\text { operation completed; the optimization models and test cases setup mechanism are presented to } \\
\text { the IAB } \\
100 \% \text { Completion: The pre-event preparation and post-event energy management optimization } \\
\text { model is completed and presented to the IAB meeting on } 1 / 3 / 2019,7 / 12 / 2019 \text {, and } 4 / 13 / 2020 \text {. }\end{array}$ \\
\hline T2.3 & $\begin{array}{l}\text { Development of solution algorithms of the pre-event optimization model. } \\
\text { Completion in Q3-FY19: A solution algorithm of the proposed pre-event proactive model has } \\
\text { been developed and tested in a small-scale system (123-node test feeder). }\end{array}$ \\
\hline $\begin{array}{l}M \\
1.2 .2\end{array}$ & $\begin{array}{l}\text { Development of solution algorithms for the refined optimization models with preliminary testing } \\
\text { results via simulation on small-scale test cases generated from subtask } 4.1 \text { and reviewed by the } \\
\text { industrial advisory board and DOE team; the resilience improvement should be at } 10 \% \text { in terms } \\
\text { of served energy and reduction of outage duration. } \\
100 \% \text { Completion: The development of the pre-event preparation optimization model is } \\
\text { complete and is presented to IAB. } \\
\text { Developed solution algorithms for the refined optimization model and the algorithm is tested on } \\
\text { small-scale test cases, with } 90 \text { minutes computation time and } 27 \% / 15 \% \text { resilience improvement } \\
\text { in served energy and reduction of outage duration. }\end{array}$ \\
\hline T2.4 & $\begin{array}{l}\text { Case studies and comparison via simulation (in Matlab or Python) using large-scale test cases } \\
\text { under different hypothetical damage scenarios to verify the effectiveness of the method and } \\
\text { refine algorithms as needed } \\
\text { Completion in Q4-FY19: A large-scale system (consisting of } 3 \text { existing test systems, EPRI ckt5 } \\
\text { system, EPRI ckt7 system, IEEE } 8500 \text { bus system) has been devised to test the performance of } \\
\text { the proposed pre-event proactive model. }\end{array}$ \\
\hline
\end{tabular}


\begin{tabular}{l|l} 
M & Development of solution algorithms for the refined optimization models with large-scale test
\end{tabular}

1.2.3 cases developed in subtask 4.2; the resilience improvement should be at $10 \%$ in terms of served energy and reduction of outage duration

$100 \%$ Completion: The solution algorithm is tested on a large-scale system (with 14,319 nodes) with 10.3 hours of computation time and $20 \% / 30 \%$ resilience improvement in served energy and reduction of outage duration.

Deliverables: Optimization model and algorithm for pre-event preparations

Subtask 2.1: State-of-the-art review of the pre-event preparation methodologies

The project team conducted a literature review on the state-of-the-art of pre-event preparation methodologies and they are leveraged to accelerate the timeline and improve the quality of the framework developed in this project. Details can be found in the Background section.

\section{Subtask 2.2: Pre-event preparation optimization model}

Model objective: The objective of the proposed model is to minimize the total cost of preparation operational cost, as follows:

$\min \sum_{\forall s} \operatorname{Pr}(s)\left(\sum_{\forall t}\left(C^{f} \sum_{\forall i} r_{i}^{f} \sum_{\varphi} P_{i, \varphi, t, s}^{G}+C^{S w} \sum_{k \in \Omega_{S W}} \gamma_{k, t, s}+\sum_{\forall \varphi} \sum_{\forall i} C_{i}^{D}(1-\right.\right.$

$\left.\left.\left.y_{i, t, s}\right) d_{i, \varphi, t}^{p}\right)\right)$

where $\left\{C^{f}, C^{s w}, C^{D}\right\}$ are the costs of fuel, switching operation, and load shedding, respectively. $\operatorname{Pr}(s)$ represents the probability of scenario $s$. The first term in the objective represents the amount of fuel generator $i$ consumes in scenario $s$ and its costs, where $r_{i}^{f}$ is the rate of fuel consumption and $P^{G}$ is the generated active power. When calculating the switching operation cost in the second term, $\gamma_{k, t, s}$ is a binary variable that is equal to 1 if switch $k$ is operated. In the third term, $y_{i, t, s}$ is a binary variable that is equal to 0 if demand $d^{p}$ is shed.

First-stage constraints: The first-stage constraints revolve around pre-allocating four critical assets that will be utilized after an extreme event: 1) mobile emergency generators (MEG); 2) mobile energy storage (MES) devices; 3) fuel; and 4) repair crews. The mobile resources can be used to restore energy for isolated areas that are not damaged, and to restore critical customers. Fuel management is critical after an extreme event to operate the emergency generators. Distributing the fuel after an extreme event may be difficult due to road conditions. As for the crews, pre-assigning them to different locations provides a faster and more organized response. The constraints for mobile sources are modeled as follows:

$\sum_{i \in \Omega_{C N}} x_{i}^{M E G}=N^{M E G}$

$\sum_{i \in \Omega_{C N}} x_{i}^{M E S}=N^{M E S}$

$x_{i}^{M E G}+x_{i}^{M E S} \leq N_{i}^{U}, \forall i \in \Omega_{C N}$

Binary variables $x^{M E G}$ and $x^{M E S}$ decide where to install the mobile devices. Constraints (2) and (3) state that the number of installed MEGs and MESs are equal to the number 
of available devices. We assume that each bus can have a limited number of the mobile unit ( $N_{i}^{U}$ ), which is enforced by (4). Define $\Omega_{G}=\Omega_{E G} \cup \Omega_{C N}$, where $\Omega_{E G}$ is the set of buses that have fuel-based generators, and $\Omega_{C N}$ is the set of candidate buses for mobile units. The fuel allocated to these buses must be limited to the amount of fuel available, as follows:

$\sum_{i \in \Omega_{G}} f_{i} \leq F^{T}$

$F_{i}^{C} \leq f_{i} \leq F_{i}^{\max }, \forall i \in \Omega_{G}$

where $f_{i}$ is the amount of fuel allocated to the generator at bus $i, F^{T}$ is the total amount of fuel the utility has, and $F_{i}^{C}$ is the amount of fuel already present for the generator at bus $i$. Constraint (5) calculates the total amount of fuel and (6) limits the amount of fuel onsite. To allocate the crews, we divide the network into different regions $\Omega_{R}$ where each region will be assigned to different crews who will conduct the repairs in that location. The crews are allocated to the regions using equations (7)-(8).

$\sum_{r \in \Omega_{R}} A_{r}=N^{C}$

$N_{r}^{C_{\min }} \leq A_{r} \leq N_{r}^{C_{\max }}, \forall r \in \Omega_{R}$

$A_{r}$ is the number of crews in region $r$, and $N^{C}$ is the total number of crews. The number of crews is limited in each region depending on the capacity of the staging locations.

Second-stage constraints: Resource decisions are made in the second stage to satisfy the problem for all possible scenarios and minimize the total cost. The second-stage constraints include the following constraints: 1) unbalanced power flow operation; 2) voltage constraints; 3) demand response; 4) generators and line flow limits; 5) reconfiguration and fault isolation; 6) BESS constraints; 7) PV system constraints; 8) repair process constraints. The PV system and repair process constraints are presented below. Details for the rest of the constraints can be found in [T2-1] and [T2-2].

PV System: In this project, we consider three types of PV systems [T2-3, T2-4, T2-5], their basic definitions are illustrated in Table B-1, detailed model are as follows:

- Type I: On-grid (grid-tied) PV $\left(\Omega_{P V}^{G}\right)$ : during an outage, the PV is switched off.

- Type II: Hybrid on-grid/off-grid PV + ESS $\left(\Omega_{P V}^{H}\right)$ : the PV system operates on-grid in normal conditions and off-grid during an outage.

- Type III: Grid-forming PV + BESS with black start capability $\left(\Omega_{P V}^{C}\right)$ : this system can restore part of the network that is not damaged if the fault is isolated.

The active power output of a PV depends on the rating of the solar cell and solar irradiance. The generated output power from the PVs is determined using the following constraints [T2-6]:

$$
\begin{array}{lc}
0 \leq P_{i, \varphi, t, s}^{P V} \leq \frac{I_{r_{i, t, s}}}{\left(1000 W / m^{2}\right)} P_{i}^{P V_{\max }} & \forall i \in \Omega_{P V} / \Omega_{P V}^{G}, \varphi, t, s \\
0 \leq P_{i, \varphi, t, s}^{P V} \leq \chi_{i, t, s} \frac{I_{r_{i, t, s}}}{\left(1000 W / m^{2}\right)} P_{i}^{P V_{\max }} & \forall i \in \Omega_{P V}^{G}, \varphi, t, s \\
\left(Q_{i, \varphi, t, s}^{P V}\right)^{2}+\left(P_{i, \varphi, t, s}^{P V}\right)^{2} \leq\left(S_{i}^{P V}\right)^{2} & \forall i \in \Omega_{P V} / \Omega_{P V}^{G}, \varphi, t, s \\
\left(Q_{i, \varphi, t, s}^{P V}\right)^{2}+\left(P_{i, \varphi, t, s}^{P V}\right)^{2} \leq \chi_{i, t, s}\left(S_{i}^{P V}\right)^{2} & \forall i \in \Omega_{P V}^{G}, \varphi, t, s
\end{array}
$$

Page 13 of 56 


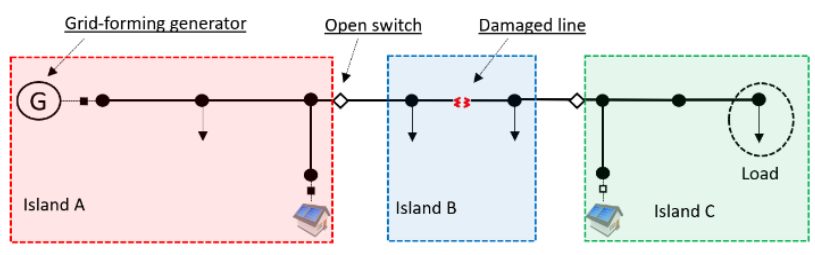

Figure 2-1. A single line diagram of a network with one damaged line.
The PVs of types $\Omega_{P V}^{G}$ and $\Omega_{P V}^{H}$ are able to disconnect from the grid and serve the on-site load. On the other hand, on-grid PVs are disconnected and the on-site load is not served by the PVs during an outage, therefore, the limit in (10) is multiplied by $\chi_{i}$ (this is known as antiislanding). $\chi_{i, t, s}$ is equal to 0 if bus $i$ is deenergized at time $t$ scenario $s$. Using advanced PV inverters, the PVs can provide reactive power support, which is constrained by (11) and (12). The connectivity constraints of the PVs are represented by constraints (13)-(17). The idea is to use virtual sources, loads, and flow to identify the energized buses in the network. In this work, we assume that the network can be restored using the grid-forming sources in $\Omega_{P V}^{C} \cup \Omega_{G} \cup \Omega_{S u b}$. A PV of type $\Omega_{P V}^{G}$ or $\Omega_{P V}^{H}$ can connect to the grid only after the PV bus is energized. Consider the network shown in Figure 2-1. Due to a damaged line, the network is divided into three islands. Island $A$ has a grid-forming generator, therefore, a microgrid is created and the PV system can participate. Island B must be isolated because of the damaged line. Island $C$ does not have any grid-forming generators; hence, it will not be active and the grid-tied PV will be disconnected.

To identify whether an island is energized

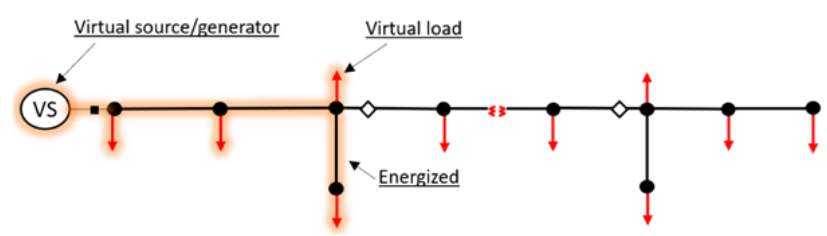

Figure 2-2. A virtual network created for the network in Figure 2-1. by grid-forming sources or not, we create a virtual network. First, each grid-forming source is replaced by a virtual source/generator with infinite capacity. Other power sources without grid-forming capability (e.g., grid-tied PVs) are removed. Also, virtual loads with a magnitude of 1 are placed on each bus, and the actual loads are removed. For example, the network shown in Figure 2-1 is transformed to the network shown in Figure 2-2. In the mathematical model, we add a power-balance equation for each virtual bus. If the virtual load at a bus is served, then that bus is energized. Therefore, for islands without grid-forming generators, all buses will be de-energized as the virtual loads in the island cannot be served. The constraints for the virtual framework are formulated as follows:

$$
\begin{array}{lr}
\sum_{\forall j \in \Omega_{P V}^{C} \cup \Omega_{G} \cup \Omega_{S u b}} v_{j, t, s}^{S}+\sum_{k \in K(., i)} v_{k, t, s}^{f}=\chi_{i, t, s}+\sum_{k \in K(i, .)} v_{k, t, s}^{f} & \forall i \in \Omega_{N}, t, s \\
-\left(u_{k, t, s}\right) M \leq v_{k, t, s}^{f} \leq\left(u_{k, t, s}\right) M & \forall k \in \Omega_{K}, t, s \\
0 \leq v_{k, t, s}^{S} \leq\left(x_{i}^{M E G}+x_{i}^{M E S}\right) M & \forall i \in \Omega_{C N}, t, s \\
\chi_{i, t, s} \geq y_{i, t, s} & \forall i \in \Omega_{N} \backslash\left\{\Omega_{P V}^{C} \cup \Omega_{G} \cup \Omega_{S u b}\right\}, t, s \\
\chi_{i, t, s}+x_{i}^{M E G}+x^{M E S} \geq y_{i, t, s} & \forall i \in \Omega_{C N}, t, s
\end{array}
$$

Page 14 of 56 
Constraint (13) is the node balance constraint for the virtual network. Virtual sources $\left(v_{i}^{S}\right)$ are connected to buses with power sources that have the capability to restore the system. The variable $v_{k}^{f}$ represents the virtual flow on line $k$ and each bus is given a load of 1 that is multiplied by $\chi_{i}$. Therefore,

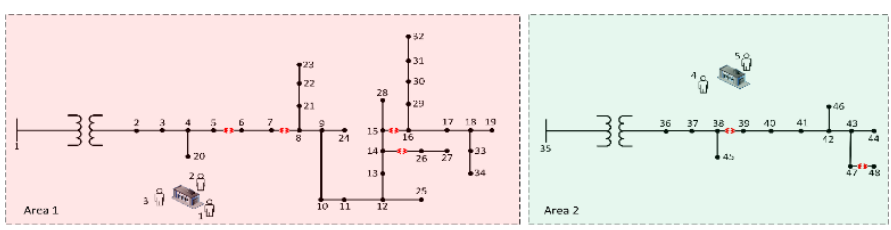

Figure 2-3. A system divided into two areas with 2 depots and 5 crews.

$\chi_{i}=1$ (bus $i$ is energized) if the virtual load can be served by a virtual source and 0 (bus $i$ is de-energized) otherwise. The virtual flow limits are defined in (14). The virtual source can be used only if a generator is installed, as enforced by (15). If bus $i$ is de-energized, then the load must be shed (16), unless bus $i$ has a local power source with a disconnect switch. Constraint (17) is similar to (16) but with the presence of mobile sources.

Repair Crews: To model the repair process, we solve an allocation problem where crews are allocated to damaged components at each time step. Crews are allocated to damaged components that are in the area they are assigned to, while the travel time is neglected. Consider the system shown in Figure 2-3, where two working areas are defined for the crews. The four damaged lines in Area 1 will be repaired by crews 1-3, while crews 4 and 5 are responsible for the two damaged lines in Area 2. Constraints (18)-(20) model the repair process of the lines.

$\sum_{\forall k \in \Omega_{D K(s)}} z_{k, t, s} \leq A_{r}$

$$
\begin{aligned}
& \forall r, t, \mathrm{~s} \\
& \forall k \in \Omega_{D K(s)}, s \\
& \forall k \in \Omega_{D K(s)}, t, s
\end{aligned}
$$$$
\begin{aligned}
& \sum_{\forall t} z_{k, t, s} \leq T_{k, s} \\
& \frac{1}{\mathcal{T}_{k, S}^{r}} \sum_{\tau=1}^{t-1} z_{k, \tau, s}-1+\epsilon \leq u_{k, t, s} \leq \frac{1}{\mathcal{T}_{k, S}^{r}} \sum_{\mathcal{T}=1}^{t-1} z_{k, \tau, s}
\end{aligned}
$$

Define $z_{k, t, s}$ as a binary variable that equals 1 if line $k$ is being repaired at time $t$ and scenario $s$, and $\Omega_{D K(s)}$ as the set of damaged lines in scenario $s$. Constraint (18) limits the number of repairs being conducted in each region according to the number of crews $\left(A_{r}\right)$ available. Constraint (19) defines the repair time for each damaged line. The line status $u_{k, t, s}$ equals 0 until the repair process is conducted for $T_{k, s}^{r}$ periods. For example, let $T_{k, s}^{r}=$ 3 , and $z_{k, t, s}=\{0,0,1,1,1,0,0\}$. Therefore, $u_{k, t, s}=\{0,0,0,0,0,1,1\}$, which is achieved by (20). For $t=6$ and $\epsilon=0.001$, constraint (20) becomes $0.668 \leq u_{k, 6, s} \leq 1$, therefore, $u_{k, 6, s}=1$.

\section{Subtask 2.3: solution algorithm}

When the number of events/scenarios is finite, a two-stage stochastic linear program can be modeled as a single large linear programming model, where each constraint in the problem is duplicated for each realization of the random data. For problems where the number of realizations is too large or infinite, the Monte Carlo sampling technique can be used to generate a manageable number of scenarios. An extensive form (EF) for the twostage stochastic program can then be defined as follows:

$\zeta=\min _{x, y_{s}} a^{T} x+\sum_{\forall s} \operatorname{Pr}(s) b_{s}^{T} y_{s}$

s.t. $\left(x, y_{s}\right) \in Q_{s}$

$\forall s$ 
where $a$ and $b_{s}$ are vectors containing the coefficients associated with the first-stage $(x)$ and second-stage $\left(y_{s}\right)$ variables in the objective, respectively. The restriction $\left(x, y_{s}\right) \in \mathcal{Q}_{s}$ represents the subproblem constraints that ensure a feasible solution. $\operatorname{Pr}(s)$ is the probability of occurrence for scenario $s$.

Rockafellar and Wets [T2-7] developed the Progressive Hedging (PH) algorithm and Watson and Woodruff adapted the algorithm [T2-8] to approximately solve stochastic mixed-integer problems. The $\mathrm{PH}$ algorithm decomposes the extensive form into scenariobased subproblems, by relaxing the non-anticipativity of the first-stage variables. Hence, for $|S|$ scenarios, the stochastic mixed-integer linear program (SMIP) is decomposed into $|S|$ subproblems. The authors in [T2-9] effectively implemented $\mathrm{PH}$ for solving the stochastic unit commitment problem. The $\mathrm{PH}$ algorithm is described in Figure 2-4 using a penalty factor $\rho$ and a termination threshold $\varepsilon$. After initializing the iteration number $\tau$, the $\mathrm{PH}$ algorithm starts by solving the subproblems with individual scenarios in Step 3. Notice that for an individual scenario, the two-stage model boils down to a single-level problem. In Step 4, the first stage solution obtained from Step 2 is aggregated to obtain the expected value $\bar{x}$. Step 5 calculates the value of the multiplier $\eta_{s}$. In Step 8, the subproblems are solved, where each subproblem is augmented with a linear term proportional to the multiplier $\eta_{s}^{\tau-1}$ and a squared two norm term penalizing the difference of $x$ from $\bar{x}^{\tau-1}$. Steps 910 repeat Steps $4-5$. The algorithm terminates once all first-stage decisions $x_{s}$ converge to a

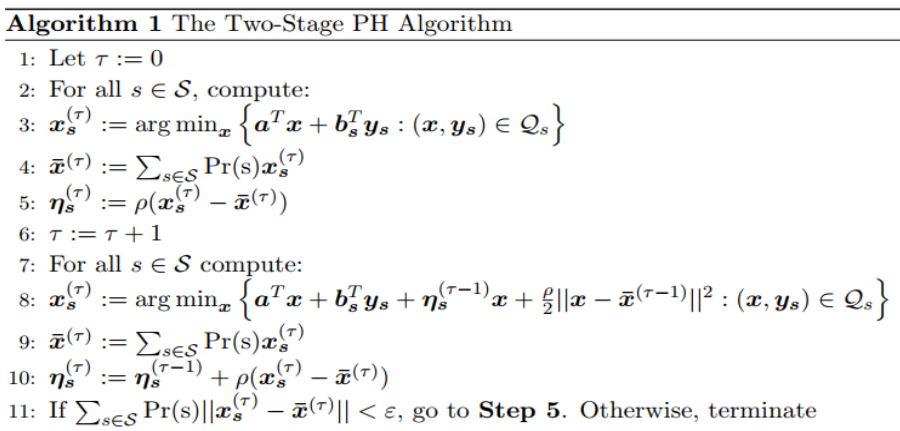

Figure 2-4. Two-stage PH Algorithm common $\bar{x}$.

\section{Subtask 2.3 and Subtask 2.4: results of small-scale and large systems}

Both small-scale systems and large-scale systems have been devised to test the performance of the proposed pre-event proactive model. The stochastic models and algorithms are implemented using the PySP package in Pyomo [T2-11]. IBM's CPLEX 12.6 mixed-integer solver is used to solve all subproblems. The experiments were performed on lowa State University's Condo cluster, whose individual blades consist of two $2.6 \mathrm{GHz} 8$-Core Intel E5-2640 v3 processors and $128 \mathrm{~GB}$ of RAM.

\section{Results of the small-scale system:}

The modified IEEE 123-bus distribution feeder [T2-10] is used as a small-scale system test case for the preparation problem. The network is modified by including 3 dispatchable DERs, 18 new switches, 5 PVs, and 2 BESSs. The pre-event model, with 5 damage scenarios under hurricane extreme weather generated in Task 4, is solved in 2 hours. The damage scenario generation is explained in Task 4 . The first-stage decision variables 
(locations of portable devices and crews) are shown in Figure 2-5. A portable generator is installed near the substation at bus 1 to act as a backup energy source in case the substation is damaged. A portable generator is installed at bus 51 to service buses 35 to 49 . A portable battery is also installed at bus 95 to serve buses 91 to 96 . Three crews are allocated to depot 2 , while 4 crews are allocated to depot 1. There are more crews at depot 1 because the lines closer to this depot have higher damage probabilities in this simulation.

To evaluate the performance of the preparation solution, we randomly generate an additional scenario and test the response of the system. The generated scenario has 8 damaged lines. Pre-event preparations using $\mathrm{PH}$ with a limited number of scenarios for the small system provided a good solution to the generated damage scenario. The computation time is within 90 minutes and the proposed model improved $27 \%$ served energy and reduced $15 \%$ outage duration compared with the base model.

\section{Results of the large-scale system:}

From Task 4, the large-scale system consists of 3 existing test systems (EPRI ckt5 system, EPRI ckt7 system, IEEE 8500 bus system) and is used as a test case for the preparation problem. The network contains 9 dispatchable DERs, 123 switches, 15 PVs, and 12 BESSs. The 9 DERs are rated at $300 \mathrm{~kW}$ and 250 kVAr. Large PVs are rated at $500 \mathrm{~kW}$. Small

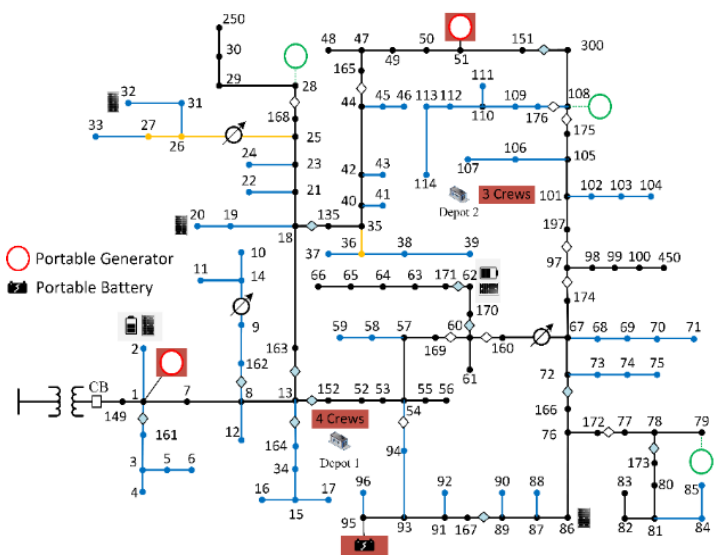

Figure 2-5. Allocation of the additional resources in the IEEE 123-bus system.

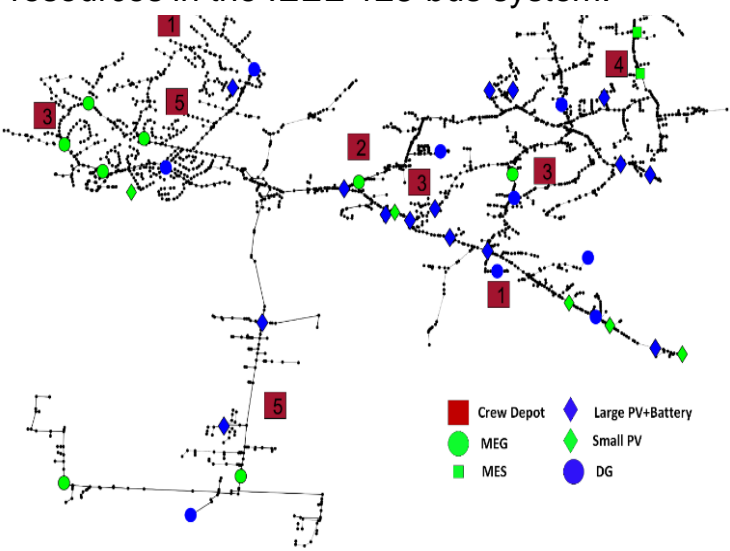

Figure 2-6. Allocation of the additional resources in the larae-scale test svstem. $\mathrm{PVs}$ are rated at $11 \mathrm{~kW} 22 \mathrm{~kW}$. The BESSs are rated at $500 \mathrm{~kW} / 4000 \mathrm{kWh}$.

1) Pre-event Preparation Results

The pre-event model, with 10 damage scenarios, is solved in 10.2 hours. The first-stage decision variables (locations of mobile energy generator (MEG), mobile energy storage (MES), and crews) are shown in Figure 2-6. 27 crews are allocated to 9 different depots. The value inside the crew depot in Figure 2-6 represents the number of crews dispatched at that depot. If more crews are dispatched at a depot, it indicates the lines closer to this depot have higher damage probabilities in this simulation.

2) Performance of the proposed model 
To evaluate the performance of the developed model, the model is compared to a typical utility approach in disaster preparation. The base case is generated by following the procedure below:

- Mobile generators are prepositioned at the substations.

- Extra mobile generators are prepositioned at high-priority loads.

- PV and battery storage are not considered

- Fuel is allocated to the mobile generators such that they can operate for at least 24 hours.

- Crews are allocated evenly between depots. In case of an odd number of crews, the location with more components will have a higher number of crews.

To compare the performance of the proposed model and the base model, we generate a random scenario and test the response of the system. The generated scenario has 103 damaged lines and they were aggregated to 34 damaged areas in Figure 2-7. Each circle represents the repair time needed for the specific damaged area considering all the aggregated lines and nodes without defined coordinates and not shown in this map. The larger circle represents a longer repair time.

We assume the substation is not receiving power from the transmission system for 6 hours. The location of the resources for the base model is intuitively placed, where we

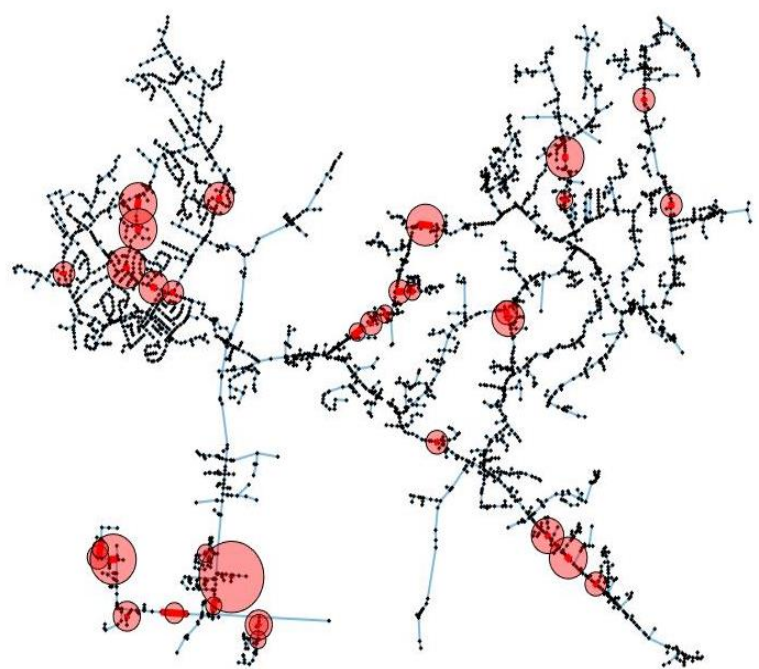

Figure 2-7. Aggregated damaged areas. assume PV systems are not participating in the restoration process.

To show the advantages of the PV systems, we test the response of the system with the proposed method and varying PV penetration levels. The total capacity of regular PV penetration can serve $33.33 \%$ load, while the high PV penetration can serve $50 \%$ load. It is observed that the increased PV penetration leads to a different allocation of additional resources.

3) Convergence Speed

The convergence metric of the progressive hedging algorithm at each iteration is expressed as the expected deviation from the mean summed across all first-stage variables and divided by the number of the first-stage variables as follows [T2-3]:

$g^{k}=\sum_{s \in S} p_{r}(s)\left\|x^{k}(s)-\bar{x}^{k}\right\|$

where $p_{r}(s)$ represents the probability of a scenario; $x^{k}(s)$ is the compact first-stage variable in a scenario $s$ at $k$ iteration; $\bar{x}^{k}$ represents the average value of the first-stage variable at $k$ iteration. 
In this project, we use the convergence metric to evaluate the convergence speed of the proposed model. At the same time, we also compare the computational speed with and without a soft-start solution. The comparison result is shown in Figure 2-8. Here we set the threshold as 0.01 . If the convergence metric reaches 0.01 , the algorithm will stop and get the optimal solution. The instance with a soft-start solution converges at the $57^{\text {th }}$ iteration and takes

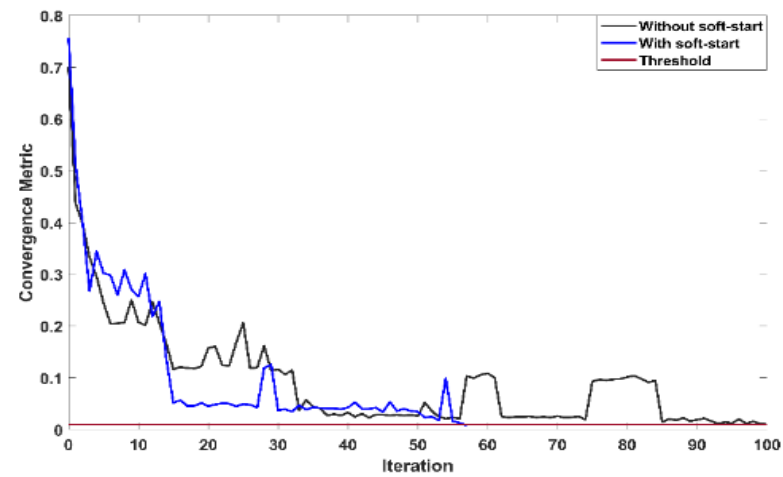

Figure 2-8. The convergence metric comparison with and without soft-start solutions. 10.2 hours. Without a soft-start solution, the instance converges at 100 iterations and takes 24.3 hours.

4) Solution Validation

To test the solution quality based on the limited generated damage scenarios, a multiple replication procedure (MRP) in [T2-2] is used to test the stability and quality of the candidate solutions. MRP is to repeat the procedure of generating 10 scenarios and solving the proposed model 10 times and constructing the confidence interval $(\mathrm{Cl})$ for the optimality gap. The one-sided $\mathrm{Cl}$ of the candidate solutions in the percentage term regarding the objective value for the optimality gap is $[0,12.48 \%]$. This small gap shows the candidate solutions are stable and of high quality.

\section{Task 3 Development of post-event operation and restoration optimization models and solution algorithms}

\begin{tabular}{|l|l|}
\hline Tasks & \multicolumn{1}{c|}{ Description } \\
\hline T3 & $\begin{array}{l}\text { Task Name: Development of post-event operation and restoration optimization models and } \\
\text { solution algorithms } \\
\text { Task Description: Develop an optimization model and solution algorithms for post-event } \\
\text { operation supported by DERs. Develop a service restoration optimization model and solution } \\
\text { algorithms to pick up loads. }\end{array}$ \\
\hline T3.1 & $\begin{array}{l}\text { State-of-the-art review of the post-event operation methodologies } \\
\text { Completion in Q1-FY19: Completed comprehensive review }\end{array}$ \\
\hline T3.2 & $\begin{array}{l}\text { Development of an optimal energy management optimization model and solution algorithms } \\
\text { for islanded operation supported by DERs after the event } \\
\text { Completion in Q3-FY19: Proposed an improved and practical post-event energy } \\
\text { management model. }\end{array}$ \\
\hline $\mathbf{M}$ & $\begin{array}{l}\text { Development of refined optimal energy management optimization model and solution } \\
\text { algorithms with intermediate testing results based on small-scale test cases; the resilience } \\
\text { improvement in terms of served energy will be at least 10\%. } \\
\text { 100\% Completion: Complete optimization model and solution algorithm development for } \\
\text { optimal energy management. } \\
\text { The algorithm is tested on a small-scale system with less than 1 hour in computation time and } \\
\text { resilience improvement ranges from 16\% to 31\% }\end{array}$ \\
\hline
\end{tabular}




\begin{tabular}{|l|l|}
\hline T3.3 & $\begin{array}{l}\text { Development of optimal restoration and load pick up optimization model and solution } \\
\text { algorithms using DERs, network reconfiguration, and repair crew } \\
\text { Completion in Q3-FY19: Developed a routing-based integrated restoration and crew dispatch } \\
\text { model. }\end{array}$ \\
\hline $\mathbf{M}$ & $\begin{array}{l}\text { Development of optimal restoration and load pick-up optimization model and solution } \\
\text { algorithms using DERs, network reconfiguration, and repair crew and intermediate testing } \\
\text { results based on small-scale test cases the resilience improvement in terms of reduction of } \\
\text { outage duration will be at least 10\%. } \\
\text { 100\% Completion: Complete the optimization model formulation for restoration and load pick } \\
\text { up. } \\
\text { Complete solution algorithm development and case studies on small-scale test cases with } \\
\text { resilience improvement ranges from 16\% to 31\% }\end{array}$ \\
\hline T3.4 & $\begin{array}{l}\text { Case studies and comparison via simulation (in Matlab or Python) using large-scale test cases } \\
\text { under given hypothetical damage scenarios to verify the effectiveness of the method and } \\
\text { refine algorithms as needed } \\
\text { Completion in Q4-FY19: case studies on a large-scale test feeder (more than 10,000 nodes) } \\
\text { are presented to verify the effectiveness of the proposed post-event energy management } \\
\text { model. The rolling horizon approach is employed to improve the computation speed. }\end{array}$ \\
\hline $\begin{array}{l}\text { M } \\
\mathbf{1 . 3 . 3}\end{array}$ & $\begin{array}{l}\text { Case studies of the solution algorithms of energy management optimization and restoration } \\
\text { optimization under large-scale test cases with three-phase multiple feeders with at least } \\
\text { 10,000 nodes generated within required computation time (5 min for energy management } \\
\text { optimization and 1 hour for restoration optimization) were completed; the resilience } \\
\text { improvement will be at least 10\% in terms of served energy and outage duration reduction. } \\
\text { 100\% Completion: The solution algorithm for optimal energy management is tested on a } \\
\text { large-scale test case. The solution algorithm for restoration optimization is tested on a large- } \\
\text { scale test system (with 14,319 nodes) with a computation time of less than 1 hour and } \\
\text { resilience improvement ranges from 30\% to 36\%. }\end{array}$ \\
\hline Deliverables: Optimization model and algorithm for post-event operations
\end{tabular}

\section{Subtask 3.1: State-of-the-art review of the post-event operation methodologies}

The project team conducted a literature review on the state-of-the-art of post-event preparation methodologies and they are leveraged to accelerate the timeline and improve the quality of the framework developed in this project. Details can be found in the Background section.

Subtask 3.2: Development of an optimal energy management optimization model and solution algorithms for islanded operation supported by DERs after the event The proposed framework for post-event energy management in the distribution network could be used by the distribution system operator (DSO) to minimize the operation cost and demand curtailment by determining the set points of dispatchable generation assets including distributed energy resources (DER) and energy storage systems (ESS), as well as exercising demand curtailment. Post-event energy management is formulated as a stochastic unbalanced OPF problem. This mixed-integer linear programming (MILP) problem could be solved using off-the-shelf solvers e.g. CPLEX and GUROBI. The uncertainties in available PV generations are captured by considering several operation scenarios with respective probabilities. The controllable variables in the proposed 
formulation are 1) the real and reactive power dispatch for DER, ESS, PV generation units, and the main distribution feeder; 2) the reactive power injection of shunt capacitors, 3 ) the primary and secondary voltage of the voltage regulators, and 4) the curtailed demand at each time step.

The proposed formulation is shown in (1)-(46). The objective (1) is to minimize the expected operation cost of the network considering the probability $\operatorname{Pr}(s)$ associated with scenario $s$ and assigned value of lost load $\left(\omega_{d}\right)$ to the demand. The first term in the objective function is the cost of energy received from the main feeder, and the second term is the operation cost of the DER units which is formulated in (2). The price of real power at time $t$ received at the distribution feeder is $\rho_{n}^{t}$. The third term is the penalty associated with the curtailed demand $d$ on phase $\varphi \in \Phi:=\{a, b, c\}$ at time $t$ in scenario $s$. The value of lost load presents the priority of the demand being served. The constraints corresponding to the distributed energy resources and network are shown in (2)-(46).

$\min \sum_{s} \operatorname{Pr}(s) \cdot\left(\sum_{n} \sum_{t} \rho_{n}^{t} \cdot \sum_{\varphi} P_{n, t}^{\varphi, s}+\sum_{t} \sum_{j} C_{j}^{t, s}+\sum_{\varphi} \sum_{t} \sum_{d \in D_{d}^{c}} \omega_{d} \cdot\left(U D_{d, t} \cdot P D_{d, t}^{\varphi}-P_{d, t}^{\varphi, s}\right)\right)$ In this section the constraints are divided into two types: 1) the energy supply and demand constraints which are the constraints corresponding to the generation and demand assets, and 2) the network constraints which are the constraints enforcing the power flow in the distribution network. The constraints (2)-(46) are discussed in detail below.

\section{1) Energy Supply and Demand Constraints:}

Distributed Generation: The fuel consumption $\left(f_{j}^{t, s}\right)$ for DER $j$ is formulated as a function of generated power $f_{j}^{t, s}=a_{j}\left(\sum_{\varphi} P_{j, t}^{\varphi, s}\right)^{2}+b_{j}\left(\sum_{\varphi} P_{j, t}^{\varphi, s}\right)+c_{j}$ where the total generated power is the sum of the power on all phases $\left(\sum_{\varphi} P_{j, t}^{\varphi, s}\right)$. Here, $C_{j}^{f u e l}$ is the price of fuel and the generation cost function for DER $j$ is written as $C_{j}^{f} \cdot a_{j}\left(\sum_{\varphi} P_{j, t}^{\varphi, s}\right)^{2}+C_{j}^{f} \cdot b_{j}\left(\sum_{\varphi} P_{j, t}^{\varphi, s}\right)+$ $C_{j}^{f} \cdot c_{j}$ which is a quadratic function and is linearized using a piecewise linearization technique. In the linearized cost curve, a marginal cost $C_{j}^{f} \cdot w_{g}$ is assigned to segment $g$ of the cost curve as shown in (2)-(4). Here, $P_{j, g}^{\max }$ is the length of each segment. The total power generated by DER $j$ is the sum of the generated power on all phases as shown in (5). The dispatched real and reactive power for DG $j\left(P_{j, t}^{\varphi, s}, Q_{j, t}^{\varphi, s}\right)$ are limited by the real and reactive power capacity of the unit $\left(P_{j, \varphi}^{\max }, Q_{j, \varphi}^{\max }\right)$ as enforced by (6) and (7). Here, the availability of the existing DER unit $(j \in N G)$ is considered by the binary parameter $U X_{j, t}$. Similar constraints are presented in (8) and (9) for the set of emergency generation units $(j \in E G)$ that are determined at the pre-disaster preparation stage.

$C_{j}^{t, s}=\sum_{g} C_{j}^{f u e l} \cdot w_{g} \cdot P_{j, g}^{t, s}$

$f_{j}^{t, s}=\sum_{g} w_{g} \cdot P_{j, g}^{t, s}$

$P_{j, g}^{t, s} \leq P_{j, g}^{\max }$

$\sum_{g} P_{j, g}^{t, s}=\sum_{\varphi} P_{j, t}^{\varphi, s}$ 


$$
\begin{array}{ll}
0 \leq P_{j, t}^{\varphi, s} \leq P_{j, \varphi}^{\max } \cdot U X_{j, t} & j \in N G \\
-Q_{j, \varphi}^{\max } \cdot U X_{j, t} \leq Q_{j, t}^{\varphi, s} \leq Q_{j, \varphi}^{\max } \cdot U X_{j, t} & j \in N G \\
0 \leq P_{j, t}^{\varphi, s} \leq P_{j, \varphi}^{\max } \cdot U X_{j, t} & j \in E G \\
-Q_{j, \varphi}^{\max } \cdot U X_{j, t} \leq Q_{j, t}^{\varphi, s} \leq Q_{j, \varphi}^{\max } \cdot U X_{j, t} & j \in E G
\end{array}
$$

Distribution Feeder: The real and reactive power provided by the distribution feeder $\left(P_{n, t}^{\varphi, s}, Q_{n, t}^{\varphi, s}\right)$ is limited by the capacity of the feeder $\left(S_{n, \varphi}^{\max }\right)$, i.e., $\left(P_{n, t}^{\varphi, s}\right)^{2}+\left(Q_{n, t}^{\varphi, s}\right)^{2} \leq U F_{n, t}$. $\left(S_{n, \varphi}^{\max }\right)^{2}$. This constraint is linearized using the circular constraint linearization method presented in [T3-1]; and therefore, replaced by (10)-(13). Here, $S_{n, \varphi}^{\max }$ is the maximum apparent power capacity of the distribution feeder on phase $\varphi$. The reactive power is limited by considering an acceptable power factor at the distribution feeder $\left(P F_{n}\right)$ and therefore, (14) is enforced. As enforced by (10) and (11), once the distribution feeder is unavailable $\left(U F_{n, t}=0\right)$, the real and reactive power dispatches are set to zero.

$-U F_{n, t} \cdot S_{n, \varphi}^{\max } \leq P_{n, t}^{\varphi, s} \leq U F_{n, t} \cdot S_{n, \varphi}^{\max }$

$-U F_{n, t} \cdot S_{n, \varphi}^{\max } \leq Q_{n, t}^{\varphi, s} \leq U F_{n, t} \cdot S_{n, \varphi}^{\max }$

$-\sqrt{2} \cdot U F_{n, t} \cdot S_{n, \varphi}^{\max } \leq P_{n, t}^{\varphi, s}+Q_{n, t}^{\varphi, s} \leq \sqrt{2} \cdot U F_{n, t} \cdot S_{n, \varphi}^{\max }$

$-\sqrt{2} \cdot U F_{n, t} \cdot S_{n, \varphi}^{\max } \leq P_{n, t}^{\varphi, s}-Q_{n, t}^{\varphi, s} \leq \sqrt{2} \cdot U F_{n, t} \cdot S_{n, \varphi}^{\max }$

$-\tan \left(\cos ^{-1} P F_{n}\right) \cdot P_{n, t}^{\varphi, s} \leq Q_{n, t}^{\varphi, s} \leq \tan \left(\cos ^{-1} P F_{n}\right) \cdot P_{n, t}^{\varphi, s}$

$P V$ unit: Three types of PV generation units are considered in this study.

For PV units of Type I i.e. $v \in V^{c}$, the available real and reactive power generation $\left(P_{v, t}^{\varphi, s}, Q_{v, t}^{\varphi, s}\right)$ for PV unit $v$ is shown in (15)-(17). The PV output power is limited by two factors. The first factor is the capacity of the PV unit $\left(P_{v}^{P V_{\max }}\right)$ as shown in (15), and the second factor is the available solar irradiance $\left(I R_{v, t}^{S}\right)$ as shown in (16). Here $A_{v}$ and $U V_{v, t}$ are the area covered by the solar PV cells and their availability respectively. The reactive power of the PV unit, provided by its inverter is between the minimum and maximum limits $\left(-Q_{v}^{\varphi, \max }, Q_{v}^{\varphi, \max }\right)$ as shown in (17). For PV units of Type II and Type III i.e. $v \in V^{G}$, the PV unit is coupled with an ESS unit. The power output of PV solar cells $\left(P_{v, t}^{\prime}{ }_{v, S}\right)$ is limited by the solar irradiance as shown in (18). The power output of the PV unit is the sum of the power output of solar PV cells and the output of the energy storage as shown in (19). In (19), $P_{d c, v}^{\varphi, t, s}$ and $P_{c, v}^{\varphi, t, s}$ are the discharging and charging power of the coupled ESS unit that is connected to PV unit $v$. The total generated power of the PV unit is limited by the capacity of the PV unit $P V_{v}^{\varphi, \max }$ as shown in (20). Here, $U V_{v, t}$ represents the availability of the PV unit $v$ at time $t$. The limits for reactive power supply are imposed by (21). As shown in (22), for the ESS unit coupled with the solar PV cell, the available energy at time $t\left(E_{v, t}^{\varphi, s}\right)$ is the sum of available energy at the previous time $\operatorname{step}\left(E_{v, t-1}^{\varphi, s}\right)$, the gained energy at time step $t\left(\eta_{c}^{v} \cdot P_{c, v}^{\varphi, t, s}\right)$, and the discharged energy at this time step $\left(\frac{P_{d c, v}^{\varphi, t s}}{\eta_{d c}^{v}}\right)$. The stored

Page 22 of 56 
energy is limited by the minimum and maximum capacity of ESS $\left(E_{v, \varphi}^{\min }, E_{v, \varphi}^{\max }\right)$ as shown in (23). The discharging and charging power $\left(P_{d c, v}^{\varphi, t, s}, P_{c, v}^{\varphi, t, s}\right)$ are limited by minimum and maximum discharging and charging power limits i.e. $P_{d c, v}^{\varphi, \min }, P_{d c, v}^{\varphi, \max }, P_{c, v}^{\varphi, \min }, P_{c, v}^{\varphi, \max }$ as shown in (24) and (25). Here $I_{d c, v}^{\varphi, t, s}$ and $I_{c, v}^{\varphi, t, s}$ are the binary discharging and charging decision variables. The charging and discharging decisions are limited by the availability of the PV unit $U V_{v, t}$ as enforced by (26). If the PV unit is not available, the charging and discharging decisions will be set to zero and the output power will be zero as enforced by (20), (21), and (26). The initial available energy for the ESS unit coupled with PV generation is enforced by (27). The availability of PV units is determined based on their type. Type II PV units are not available after the outage while Type III PV units will be connected to the grid in contingencies.

$$
\begin{aligned}
& P_{v, t}^{\varphi, s} \leq P_{v}^{P V_{\max }} \cdot U V_{v, t} \quad v \in V^{c} \\
& P_{v, t}^{\varphi, s} \leq\left(\frac{1}{3}\right) \cdot A_{v} \cdot I R_{v, t}^{S} \cdot U V_{v, t} \quad v \in V^{c} \\
& -Q_{v}^{\varphi, \max } \cdot U V_{v, t} \leq Q_{v, t}^{\varphi, s} \leq Q_{v}^{\varphi, \max } \cdot U V_{v, t} \quad v \in V^{c} \\
& P_{v, t}^{\prime \varphi, s} \leq\left(\frac{1}{3}\right) \cdot A_{v} \cdot I R_{v, t}^{S} \cdot U V_{v, t} \quad v \in V^{G} \\
& P_{v, t}^{\varphi, s}=P_{v, t}^{\prime \varphi, s}+P_{d c, v}^{\varphi, t, s}-P_{c, v}^{\varphi, t, s} \quad v \in V^{G} \\
& P_{v, t}^{\prime \varphi, s}+P_{d c, v}^{\varphi, t, s}-P_{c, v}^{\varphi, t, s} \leq P_{v}^{P V_{\max }} \cdot U V_{v, t} \quad v \in V^{G} \\
& -Q_{v}^{\varphi, \max } \cdot U V_{v, t} \leq Q_{v, t}^{\varphi, s} \leq Q_{v}^{\varphi, \max } \cdot U V_{v, t} \quad v \in V^{G} \\
& E_{v, t}^{\varphi, s}=E_{v, t-1}^{\varphi, s}+\eta_{c}^{v} \cdot P_{c, v}^{\varphi, t, s}-\frac{P_{d c, v}^{\varphi, t s}}{\eta_{d c}^{v}} \quad v \in V^{G} \\
& E_{v, \varphi}^{\min } \leq E_{v, t}^{\varphi, s} \leq E_{v, \varphi}^{\max } \quad v \in V^{G} \\
& P_{d c, v}^{\varphi, \min } \cdot I_{d c, v}^{\varphi, t, s} \leq P_{d c, v}^{\varphi, t, s} \leq P_{d c, v}^{\varphi, \max } \cdot I_{d c, v}^{\varphi, t, s} \quad v \in V^{G} \\
& P_{c, v}^{\varphi, \min } \cdot I_{c, v}^{\varphi, t, s} \leq P_{c, v}^{\varphi, t, s} \leq P_{c, v}^{\varphi, \max } \cdot I_{c, v}^{\varphi, t, s} \quad v \in V^{G} \\
& I_{c, v}^{\varphi, t, s}+I_{d c, v}^{\varphi, t, s} \leq U V_{v, t} \quad v \in V^{G} \\
& E_{v, 0}^{\varphi, s}=E_{v, \text { ini }}^{\varphi, s} \quad v \in V^{G}
\end{aligned}
$$

Energy Storage Systems: The constraints for battery energy storage are presented in (28)-(33). Here $U_{m}^{t}$ is the availability of energy storage $m, E_{m, t}^{\varphi, s}$ is the available energy stored in ESS $m, P_{c, m}^{\varphi, t, s}$ the charging dispatch, $P_{d c, m}^{\varphi, t, s}$ is the discharging dispatch, $\eta_{c}^{m}$ is the charging efficiency, and $\eta_{d c}^{m}$ is the discharging efficiency. These constraints are similar to the constraints presented for the ESS coupled with the solar PV generation (22)-(27).$$
\begin{aligned}
& P_{d c, m}^{\varphi, m i n} \cdot I_{d c, m}^{\varphi, t, s} \leq P_{d c, m}^{\varphi, t, s} \leq P_{d c, m}^{\varphi, m a x} \cdot I_{d c, m}^{\varphi, t, s} \\
& P_{c, m}^{\varphi, m i n} \cdot I_{c, m}^{\varphi, t, s} \leq P_{c, m}^{\varphi, t, s} \leq P_{c, m}^{\varphi, m a x} \cdot I_{c, m}^{\varphi, t, s} \\
& I_{c, m}^{\varphi, t, s}+I_{d c, m}^{\varphi, t, s} \leq U_{m}^{t}
\end{aligned}
$$ 
$E_{m, t}^{\varphi, s}=E_{m, t-1}^{\varphi, s}+\eta_{c}^{m} \cdot P_{c, m}^{\varphi, t, s}-\frac{P_{d c, m}^{\varphi, t, s}}{\eta_{d c}^{m}}$

$E_{m, \varphi}^{\min } \leq E_{m, t}^{\varphi, s} \leq E_{m, \varphi}^{\max }$

$E_{m, 0}^{\varphi, s}=E_{m, \text { ini }}^{\varphi, s}$

Demand: The controllable demands, $\left(d \in D_{d}^{c}\right)$ are curtailed when they cannot be served. Therefore, the served real and reactive demand $\left(P_{d, t}^{\varphi, s}, Q_{d, t}^{\varphi, s}\right)$ is limited by the total demand $\left(P D_{d, t}^{\varphi}, Q D_{d, t}^{\varphi}\right)$ as shown in (34) and (35). Here, $U D_{d, t}$ represents the availability of demand.

$P_{d, t}^{\varphi, s} \leq P D_{d, t}^{\varphi} \cdot U D_{d, t}$

$d \in D_{d}^{c}$

$Q_{d, t}^{\varphi, s} \leq Q D_{d, t}^{\varphi} \cdot U D_{d, t}$

$d \in D_{d}^{c}$

Capacitor Constraints: The reactive power supplied by the capacitor is limited by the nameplate of the equipment as enforced by (36).

$0 \leq Q_{c, t}^{\varphi, s} \leq Q_{c}^{\max }$

\section{2) Network Constraints:}

Branch Flow Constraints: The real and reactive power transmitted through branch $l$ $\left(P L_{l, t}^{\varphi, s}, Q L_{l, t}^{\varphi, s}\right)$ is limited by the apparent power capacity of the branch $\left(S L_{\varphi, l}^{\max }\right)$ as shown in (37) and (38). It is worth noting that a branch $l$ includes the distribution lines, regulators, and transformers connected between two nodes. Here, $U Y_{l, t}$ represents the availability of the branch and $p_{l}^{\varphi} \in\{0,1\}$ is a binary parameter that shows the existence of phase $\varphi$ on branch $l$. The complex power flow in a branch should satisfy $\left(P L_{l, t}^{\varphi, s}\right)^{2}+\left(Q L_{l, t}^{\varphi, s}\right)^{2} \leq p_{l}^{\varphi}$. $U Y_{l, t} \cdot\left(S L_{\varphi, l}^{\max }\right)^{2}$. This constraint is linearized using the circular constraint linearization method presented in [T3-1] and therefore replaced by (39), (40).

$-U Y_{l, t} \cdot p_{l}^{\varphi} \cdot S L_{\varphi, l}^{\max } \leq P L_{l, t}^{\varphi, s} \leq U Y_{l, t} \cdot p_{l}^{\varphi} \cdot S L_{\varphi, l}^{\max }$

$-U Y_{l, t} \cdot p_{l}^{\varphi} \cdot S L_{\varphi, l}^{\max } \leq Q L_{l, t}^{\varphi, S} \leq U Y_{l, t} \cdot p_{l}^{\varphi} \cdot S L_{\varphi, l}^{\max }$

$-\sqrt{2} \cdot U Y_{l, t} \cdot p_{l}^{\varphi} \cdot S L_{\varphi, l}^{\max } \leq P L_{l, t}^{\varphi, S}+Q L_{l, t}^{\varphi, s} \leq \sqrt{2} \cdot U Y_{l, t} \cdot p_{l}^{\varphi} \cdot S L_{\varphi, l}^{\max }$

$-\sqrt{2} \cdot U Y_{l, t} \cdot p_{l}^{\varphi} \cdot S L_{\varphi, l}^{\max } \leq P L_{l, t}^{\varphi, s}-Q L_{l, t}^{\varphi, S} \leq \sqrt{2} \cdot U Y_{l, t} \cdot p_{l}^{\varphi} \cdot S L_{\varphi, l}^{\max }$

Voltage Regulator Constraints: A single-phase voltage regulator is represented as an ideal transformer in series with a leakage impedance and a three-phase voltage regulator is composed of three single-phase voltage regulators [T3-2]. Voltage regulators and transformers are considered as branches in distribution networks and Kirchhoff voltage law could be applied to these elements as discussed in the next section. For transformers with fixed tap settings, the impedance of the branch with a transformer is known. For the voltage regulators with variable tap settings, it is assumed that the internal leakage impedance is not affected by the tap ratio and the relationship between the voltage magnitudes on the primary side (bus $b$ ) and the secondary side (bus $k$ ) of the voltage regulator is expressed by (41), (42); where, $M$ is a large number [T3-2]. Here. The vectors $\boldsymbol{a}_{\text {min }}, \boldsymbol{a}_{\text {max }} \in \mathbb{R}^{3 \times 1}$ are the minimum and maximum ratios between primary and secondary 
phase voltages of the voltage regulator respectively. Here, $\boldsymbol{U}_{b, t}^{s}=$ $\left[\left(V_{b, t}^{a, s}\right)^{2}\left(V_{b, t}^{b, s}\right)^{2}\left(V_{b, t}^{c, s}\right)^{2}\right]^{T}$ is the vector of squared phase voltage magnitude for bus $b$ at time $t$ in scenario $s$. The availability of the voltage regulator is presented by binary parameter $U R_{r, t}$. Furthermore, $\boldsymbol{p}_{r}$ is the vector of binary parameters that represent the existence of phases on voltage regulator $r$. If the regulator is a three-phase regulator then $\boldsymbol{p}_{r}=\left[\begin{array}{lll}1 & 1 & 1\end{array}\right]^{T}$.

$-\left(2-U R_{r, t}-\boldsymbol{p}_{r}\right) \cdot M \leq \boldsymbol{a}_{\max , r}^{2} \cdot \boldsymbol{U}_{b, t}^{s}-\boldsymbol{U}_{k, t}^{s} \quad b \in R_{f}^{r}, k \in R_{t}^{r}$

$\boldsymbol{a}_{m i n, r}^{2} \cdot \boldsymbol{U}_{b, t}^{s}-\boldsymbol{U}_{k, t}^{s} \leq\left(2-U R_{r, t}-\boldsymbol{p}_{r}\right) \cdot M \quad k \in R_{t}^{r}, b \in R_{f}^{r}$

Kirchhoff voltage and current constraints: The nodal real and reactive power balance is formulated in (43) and (44). Here, $N_{b}$ is the set of feeders connected to bus $b, M_{b}$ is the set of energy storage units connected to bus $b, C_{b}$ is the set of shunt capacitors connected to bus $b, G_{b}$ is the set of DER units connected to bus $b, V_{b}$ is the set of PV units connected to bus $b, D_{b}$ is the set of demands connected to bus $b, L_{f, b}$ is the set of branches from bus $b, L_{t, b}$ is the set of branches to bus $b$. Assuming that the voltages on buses are nearly balanced, and the loss is small compared to the power flow in the branch, the power flow in the branch $l$ between buses $b$ and $k$ is formulated by (45), (46) where $\boldsymbol{S} \boldsymbol{L}_{l, t}^{S}$ is the apparent power flowing in the branch $l$ that includes the apparent power on each phase of the branch $l$. As shown in [T3-2], the matrix $\widetilde{\boldsymbol{Z}_{l}}$ is $\boldsymbol{A} \odot \boldsymbol{Z}_{l}$ where $\boldsymbol{Z}_{l}$ is the impedance matrix of branch $l$ in which its elements are complex numbers; $\boldsymbol{A}$ is the phase shift matrix; and $\odot$ is the element-wise product. Here $L_{f}^{l}$ and $L_{t}^{l}$ are the set of buses on the sending and receiving ends of the branch $l$ and $\boldsymbol{p}_{l}$ is the vector of binary entries for branch $l$ to represent the phases $\boldsymbol{p}_{l} \in\{0,1\}^{3 \times 1}$. The big-M method is used in (45)-(46) to ensure that the equality constraints are only applied for available branches.

$\sum_{n \in N_{b}} P_{n, t}^{\varphi, s}+\sum_{j \in G_{b}} P_{j, t}^{\varphi, s}+\sum_{m \in M_{b}}\left(P_{d c, m}^{\varphi, t, s}-P_{c, m}^{\varphi, t, s}\right)+\sum_{l \in L_{t, b}} P L_{l, t}^{\varphi, s}-$

$\sum_{l \in L_{f, b}} P L_{l, t}^{\varphi, s}+\sum_{v \in V_{b}} P_{v, t}^{\varphi, s}-\sum_{d \in D_{b}} P_{d, t}^{\varphi, s}=0$

$\sum_{n \in N_{b}} Q_{n, t}^{\varphi, s}+\sum_{j \in G_{b}} Q_{j, t}^{\varphi, s}+\sum_{m \in M_{b}} Q_{m}^{\varphi, t, s}+\sum_{l \in L_{t, b}} Q L_{l, t}^{\varphi, s}-$

$\sum_{l \in L_{f, b}} Q L_{l, t}^{\varphi, s}+\sum_{c \in C_{b}} Q_{c, t}^{\varphi, s}+\sum_{v \in V_{b}} Q_{v, t}^{\varphi, s}-\sum_{d \in D_{b}} Q_{d, t}^{\varphi}=0$

$\boldsymbol{U}_{b, t}^{s}-\boldsymbol{U}_{k, t}^{s}+\widetilde{Z_{l}} \cdot\left(\boldsymbol{S} L_{l, t}^{s}\right)^{*}+\widetilde{\boldsymbol{Z}}_{l}^{*} \cdot \boldsymbol{S L}_{l, t}^{s} \leq M \cdot\left(1-U Y_{l, t}\right) \cdot \boldsymbol{p}_{l} \quad b \in L_{f}^{l}, k \in L_{t}^{l}$

$-M \cdot\left(1-U Y_{l, t}\right) \cdot \boldsymbol{p}_{l} \leq \boldsymbol{U}_{b, t}^{s}-\boldsymbol{U}_{k, t}^{s}+\widetilde{Z_{l}} \cdot\left(\boldsymbol{S} L_{l, t}^{s}\right)^{*}+\widetilde{\boldsymbol{Z}}_{l}^{*} \cdot \boldsymbol{S} \boldsymbol{L}_{l, t}^{s} \quad b \in L_{f}^{l}, k \in L_{t}^{l}$

Subtask 3.3: Development of optimal restoration and load pick up optimization model and solution algorithms using DERs, network reconfiguration, and repair crew

In Subtask 3.3, we have developed a synthetic model for enhancing post-event grid resilience by integrating distribution system restoration and crew dispatch problems. The developed model can achieve seamless coordination among multiple service restoration tasks such as switch operation, crew dispatch, and component repair. 
In a conventional outage management system (OMS), the distribution system restoration (DSR) module and crew dispatch module function separately with limited coordination. If crews are not properly dispatched to perform the designated tasks, system operators must revise the restoration plan repeatedly, otherwise, it will take more time to restore the power for affected customers. Therefore, an integrated model that can achieve seamless coordination between DSR and crew dispatch will be preferred by the system operators. The developed model can generate optimal, coordinated, and secure switching and repair sequences. In addition to considering various operational constraints such as power balance and voltage limits, the developed model takes into account other critical constraints to ensure crew safety and operational logistics. In addition, the developed model is formulated as a MILP problem to ensure solution optimality.

The integrated restoration and crew dispatch model is based on an innovative routing model, which is developed for the first time by the project team. The basic idea is to mathematically formulate energization current and crew as travel agents, then formulate the restoration problem and repair problem using state-of-art routing models considering their interdependency constraints.

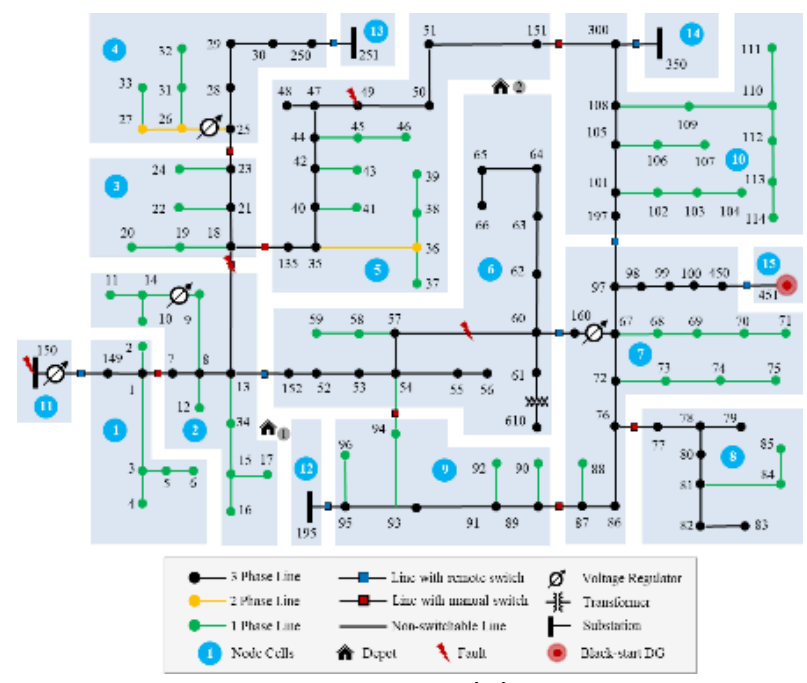

(a)

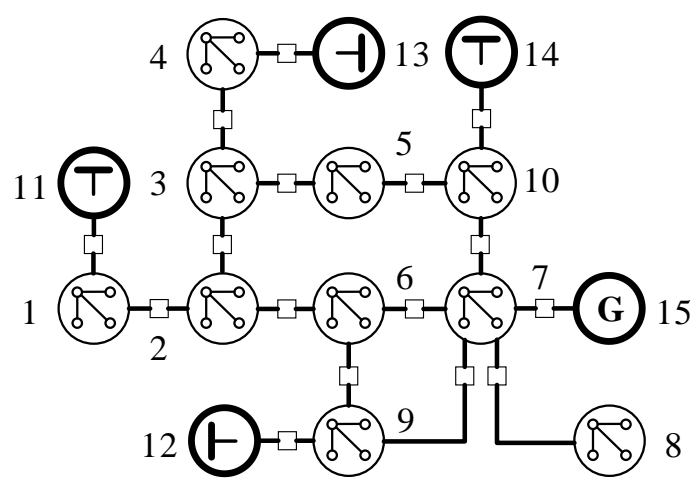

(b)

Figure 3-1. Concept of node cell. (a): Modified IEEE 123 node test feeder. (b): Node cell representation of IEEE 123 node test feeder.

1) Concept of Node Cell

The electric power system can be grouped into a set of node cells, which can be defined as a group of system components interconnected by non-switchable lines. Multiple node cells are interconnected through switches. All the components within a node cell can be energized at once by closing any one of the switches connected to it. Figure 3-1 shows the concept of a node cell to represent the IEEE 123 node test system, which can be 
significantly reduced to a simplified model to be processed in the developed restoration and crew dispatch model.

2) Energization Agent, Energization Path, Routing Table, and Arrival Time Table The idea of the energization agent is based on the fact that, during the service restoration process, the electricity travels through the feeders from the sources (e.g., substations) to energize downstream customers. The switching sequences for the switches determine the energization paths, that is, the routes along which the electricity should travel. In this sense, the DSR problem can be formulated as a routing problem by assuming each energization sequence to be an energization path, and an energization agent travels along the energization path. The difference between the aforementioned model and the traditional routing model is that, instead of assigning a fixed number of travel agents in advance, the energization agent can "split" into multiple energization agents when multiple downstream lines need to be energized, as shown in Figure 3-2.

We assume that there are three types of travel agents: the operation agent $(O A)$, repair agent (RA), and energization agent (EA). For each type of agent, we can model its activity using a graph $G(\mathcal{N}, \mathcal{E})$ with $\mathcal{N}$ as the set of nodes that the agent may visit, and $\varepsilon$ as the set of paths that the agent may travel. Note the routing table and the arrival time table has different definitions in restoration and repair models. They can be used to represent the energization sequence and pick-up time for a cell in the restoration model, and the travel sequence and arrival time for a repair crew to the destination in the repair model. As shown in Fig, $\mathcal{N}$ represents the depots and manual switches for $O A$, the depots and faulted components for RA, and the substations and node cells for EA.

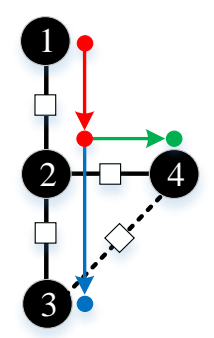

(a)

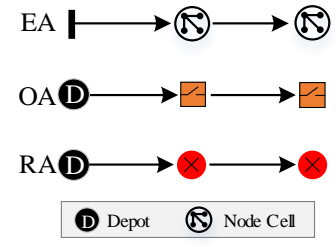

(b)

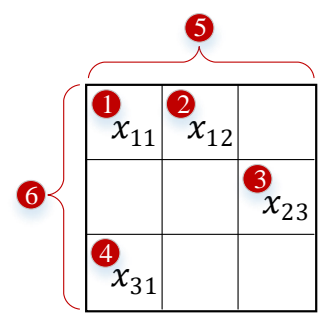

(c)

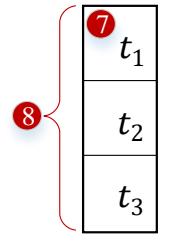

(d)

Figure 3-2. Concept of energization agent, travel crew agent, routing table, and arrival time table. (a): Energization agent travels from cell1 to cell 2 (i.e., the red route), through the switch, and the energization path splits into two paths (i.e., the green route from cell 2 to cell 4 , and the blue route from cell 2 to cell 3 ) to energize both cell 3 and cell 4 . (b): travel agents considered in the model. (c): routing table with decision variables that can describe the routing behaviors. (d): arrival time table specifying the arrival time for the travel agent to arrive at each node.

Several types of interdependency are identified between service restoration, crew for operating switches, and crew for repair, as summarized in Table 3-1. These interdependencies can be easily formulated as mathematical constraints. 
Table 3-1. Interdependency between service restoration, crew for operating switches, and crew for repair

\begin{tabular}{|c|c|c|c|c|}
\hline \multicolumn{3}{|c|}{ "Domain } & \multirow[b]{2}{*}{ Interdependence Description } & \multirow[b]{2}{*}{$\begin{array}{l}\text { Partial Variables and } \\
\text { Constraints }\end{array}$} \\
\hline$D S R$ & $\begin{array}{l}\text { Crew for } \\
\text { Operating } \\
\text { Switch }\end{array}$ & $\begin{array}{l}\text { Crew } \\
\text { for } \\
\text { Repair }\end{array}$ & & \\
\hline$\sqrt{ }$ & $\sqrt{ }$ & & $\begin{array}{l}\text { A crew operates a manually operated switch to } \\
\text { energize components. }\end{array}$ & $\begin{array}{l}x_{i j}^{O} \in\{0,1\}: \text { A crew can operate } j \\
\text { (travel from } i \text { to } j \text { ), if } j \text { is a manual } \\
\text { switch. Otherwise, } x_{i j}^{O}=0\end{array}$ \\
\hline & $\sqrt{ }$ & $\sqrt{ }$ & $\begin{array}{l}\text { A damaged switch can be operated only after } \\
\text { being repaired. }\end{array}$ & $\begin{array}{l}t_{j}^{O}>t_{j}^{R}+T_{j}^{R P}: \text { Operation time } \\
\text { (arrival time at } j \text { from } i \text { ) should be } \\
\text { later than the repaired time. }\end{array}$ \\
\hline$\sqrt{ }$ & & $\sqrt{ }$ & $\begin{array}{l}\text { A faulted component can be energized only after } \\
\text { being repaired. }\end{array}$ & $\begin{array}{l}t_{j}^{E}>t_{j}^{R}+T_{j}^{R P}: j \text { is a faulted } \\
\text { component }\end{array}$ \\
\hline$\sqrt{ }$ & $\sqrt{ }$ & $\sqrt{ }$ & $\begin{array}{l}\text { To repair a faulted component, the component } \\
\text { should be isolated by opening } \\
\text { upstream/downstream switches to ensure crew } \\
\text { safety. A switch cannot be energized when an } \\
\text { operation crew is in the process of operating it. }\end{array}$ & $\begin{array}{l}t_{j}^{R}+T_{j}^{R P}>\max \left\{t_{i}^{E}, t_{j}^{E}, t_{k}^{E}\right\}: j \text { is the } \\
\text { component to be repaired }\end{array}$ \\
\hline
\end{tabular}

In addition, a set of constraints are defined to describe the "behavior" of each type of travel agent, as summarized in Table 3-2.

Table 3-2. Route table constraints for different travel agents

\begin{tabular}{|c|c|c|c|}
\hline \multirow{2}{*}{ Route Table Constraints } & \multicolumn{3}{|c|}{ Travel Agents Defined in the Universal Routing Model } \\
\hline & $O A$ & \begin{tabular}{|r|}
$R A$ \\
\end{tabular} & $E A$ \\
\hline $\begin{array}{l}\text { Each type of agent should travel } \\
\text { starting only from the } \\
\text { depot/substation. }\end{array}$ & $\begin{array}{r}x_{i i}^{O}=1, \forall i \in \mathcal{D}^{o} \\
x_{i i}^{O}=0, \forall i \in \mathcal{M}^{o}\end{array}$ & $\begin{array}{l}x_{i i}^{R}=1, \forall i \in \mathcal{D}^{R} \\
x_{i i}^{R}=0, \forall i \in \mathcal{F}^{R}\end{array}$ & $\begin{array}{c}x_{i i}^{E}=1, \forall i \in \mathcal{G}^{E} \\
x_{i i}^{E}=0, \forall i \in \mathcal{C}^{E} \backslash \mathcal{G}^{E}\end{array}$ \\
\hline $\begin{array}{l}\text { Each type of agent should not } \\
\text { go back to the depot/substation. } \\
\text { An EA can travel from one cell } \\
\text { to another cell only through the } \\
\text { existing switches. }\end{array}$ & $\begin{array}{r}x_{i j}^{O}=0, \forall i, j \in \mathcal{D}^{O}, i \neq j \\
(7)\end{array}$ & $x_{i j}^{R}=0, \forall i, j \in \mathcal{D}^{R}, i \neq j$ & $\begin{array}{r}\sum_{h=1, h \neq i}^{n^{E}} x_{h i}^{E}=0, \forall i \in \mathcal{G}^{E} \\
(9) \\
x_{i j}^{E}=x_{j i}^{E}=0, \forall(i, j) \notin \mathcal{B}^{E} \\
(10)\end{array}$ \\
\hline $\begin{array}{l}\text { Each possible route can be } \\
\text { visited no more than once. }\end{array}$ & $\begin{array}{r}x_{i j}^{O}+x_{j i}^{O} \leq 1, \forall i, j \in \mathcal{D}^{O} \cup \\
\mathcal{M}^{O}\end{array}$ & $\begin{array}{r}x_{i j}^{R}+x_{j i}^{R} \leq 1, \forall i, j \in \mathcal{D}^{R} \cup \mathcal{F}^{R} \\
(12)\end{array}$ & $\begin{array}{r}x_{i j}^{E}+x_{j i}^{E} \leq 1, \forall(i, j) \in \mathcal{B}^{E} \\
(13)\end{array}$ \\
\hline $\begin{array}{l}\text { The total number of agents } \\
\text { dispatched out of each OA or } \\
\text { EA depot cannot exceed the } \\
\text { capacity of that depot. }\end{array}$ & $\sum_{j=1, j \neq i}^{n^{o}} x_{i j}^{O} \leq n_{\text {cap, } i}^{O}, \quad \forall i \in$ & $\begin{array}{cc}\sum_{j=1, j \neq i}^{n^{R}} x_{i j}^{R} \leq n_{\text {cap, } i}^{R}, & \forall i \in \\
\mathcal{D}^{R} & (15)\end{array} \mid$ & - \\
\hline $\begin{array}{l}\text { For each type of agent, each } \\
\text { destination can be visited by at } \\
\text { most one agent. For OA and } \\
\text { RA, an agent should leave or } \\
\text { stay at the visited destination. } \\
\text { For EA, an agent leaving a } \\
\text { visited destination can split into } \\
\text { multiple agents. }\end{array}$ & $\begin{array}{r}\sum_{j=1}^{n^{o}} x_{i j}^{o} \leq \sum_{h=1}^{n^{o}} x_{h i}^{O} \leq 1, \forall i \in \\
\mathcal{M}^{o}(16)\end{array}$ & $\begin{array}{r}\sum_{j=1}^{n^{R}} x_{i j}^{R} \leq \sum_{h=1}^{n^{R}} x_{h i}^{R} \leq 1, \forall i \in \\
\mathcal{F}^{R}(17)\end{array}$ & $\begin{array}{r}\sum_{h=1}^{n^{E}} x_{h i}^{E} \leq 1, \forall i \in \mathcal{C}^{E} \\
(18) \\
\sum_{j=1}^{n^{E}} x_{i j}^{E} \leq n^{E} \sum_{h=1}^{n^{E}} x_{h i}^{E}, \forall i \in \\
\mathcal{C}^{E}(19)\end{array}$ \\
\hline
\end{tabular}


The developed model also considers other operational constraints to ensure the feasibility of the generated restoration solution, such as radial topology constraints, voltage constraints, line capacity constraints, and DER capacity constraints.

Due to the space limit, we present the solution of a sample scenario with PV penetration being $72 \%$. Figure 3-3 shows the Percentage of total restored load along time during restoration. The light green

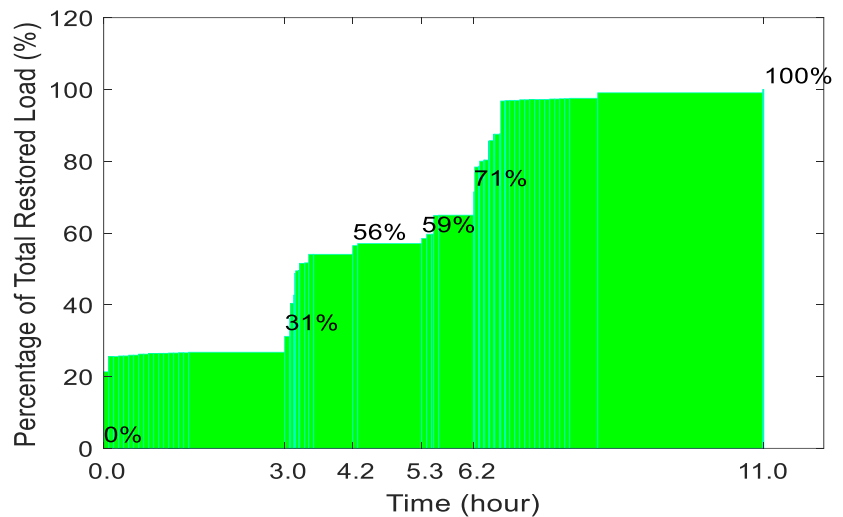

Figure 3-3. Percentage of total restored load along time during restoration lines represent the switch operations at a specific time. It can be observed that at the beginning of the restoration, there is around $20 \%$ of the load served by DERs, MEG, MES, and Type III PVs, which can operate in grid-forming mode and pick up the loads immediately. A set of switching operations can be observed for the first 1.5 hours, which represents the initial stage of restoration - reconfiguring the system to pick up the loads that are not affected by the damaged components. The rest of the restoration is an integrated process of switch operation and repair. As crews are dispatched to repair the damaged components, the associated loads will no longer be affected and can be picked up, following an optimal order determined by the restoration algorithm.

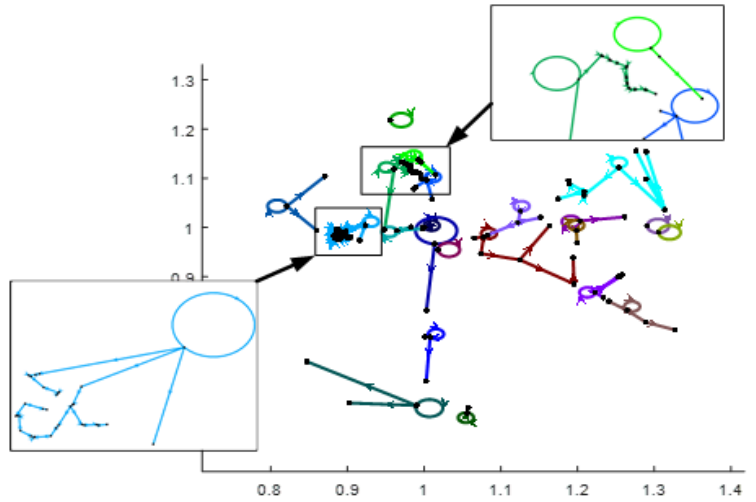

Figure 3-4. Percentage of total restored load along time during restoration

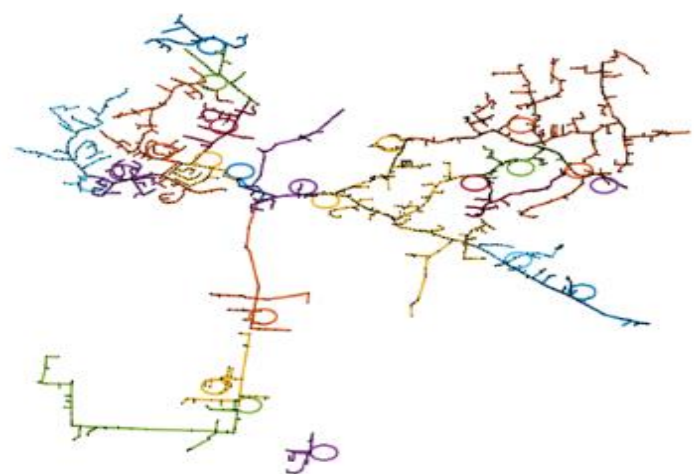

Figure 3-5. Single-line diagram of energized system. 
Figure 3-4 shows the energization sequence for the electric power network. Each circle represents a DER with black start capability. The arrows coming out of the circle represent the energization currents. Each black dot represents a block introduced in previous reports. It can be observed that the system was fully energized and divided into multiple islands. Figure 3-5 shows the single-line diagram of energized test system supported by all the power sources. The system was partitioned into multiple islands that are labeled by different colors. Note that each island contains one power source with black start capability and multiple switches and power sources without black start capability.

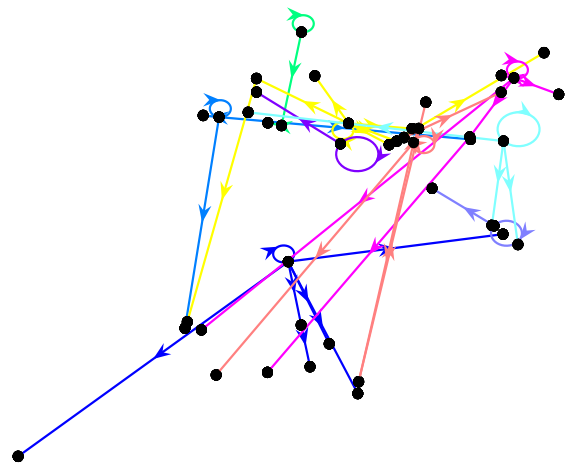

Figure 3-6. Dispatch map for repair crews Figure 3-6 shows the dispatch sequence for repair crews. The dispatch sequence is mapped on the figure according to the coordinates of damaged components. Each circle represents a depot that is hosting the crews. The crews that come out of different depots are labeled by different colors. The total number of crews dispatched out of a depot is limited by the pre-event preparation solution provided by ISU.

The project team performed a case study to verify that all damages will be repaired after loads are restored using our previously developed algorithm. As shown in Figure 3-7, the switching for restoration is completed at the 9th hour, but the repair process is completed at around the 36th hour. This is because the crews, after restoring all the loads, are dispatched to repair all the

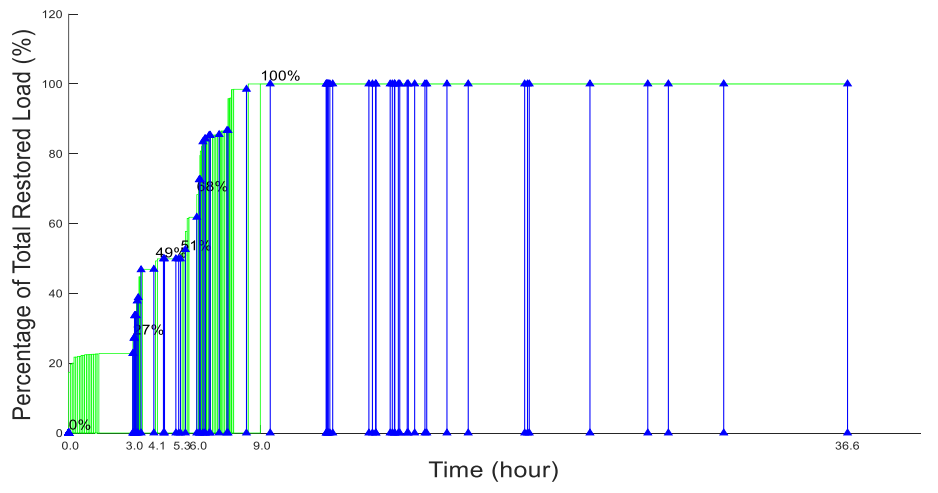
damaged PVs that consume additional time.

Figure 3-7. Switching time (green) and repair completion time (blue)

\section{Subtask 3.4: Case studies} Simulation on IEEE 123-bus System 
We leveraged the proposed formulation to perform energy management in the modified IEEE 123 -bus systems. The topology of the network is shown in Figure 3-8. The capacity of PV1-PV12 units is 50,100 , 50, 100, 50, 100, 50, 100, 50, 50, 50, and $50 \mathrm{~kW}$ respectively. PV4, PV6, and PV12 are Type III units, and PV8 is Type II. The rest of the PV units are Type I. The uncertainty in PV generation is taken into consideration. The forecast error for solar irradiance is considered by a normal distribution function in which the mean is the

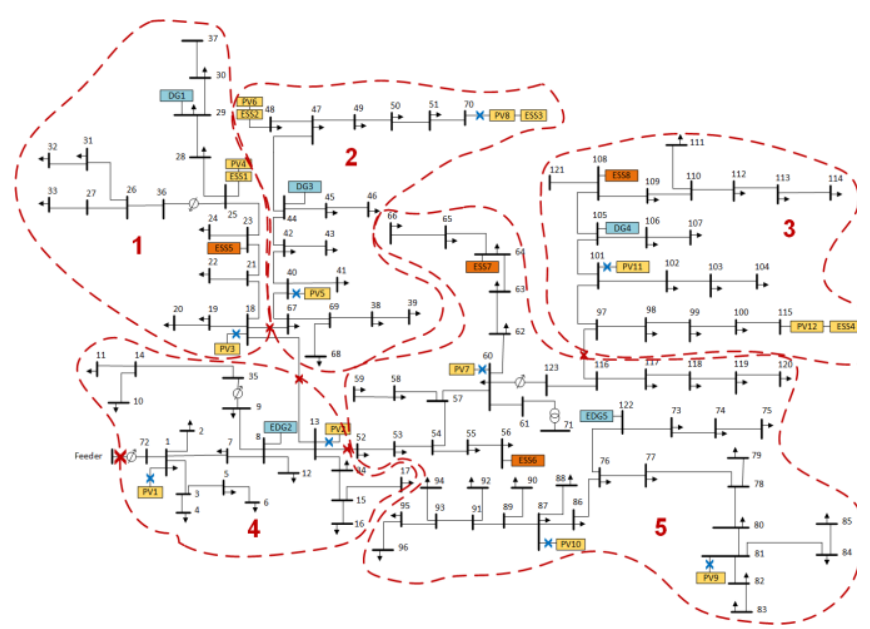

Fig. 3-8. IEEE-123 bus system considering the default status of normally closed and open switches and the feeder and branch outages.

forecasted solar irradiance, and the standard deviation is progressively increasing by $0.3 \%$ for every 5 minutes. Five scenarios including the forecasted scenario with equal probabilities were considered. The simulation is performed on a PC with an Intel Core i7 processor, and $32 \mathrm{~GB}$ of memory with CPLEX 12.8.0.

In case of an outage in the main feeder and distribution branches shown in Figure 3-7, the expected operation cost is $\$ 515890.174$, and the total expected demand curtailment is $12887.722 \mathrm{kWh}$. In this case, $20.6 \%$ of the load is served in the operation horizon. If there is no PV generation in the network, the operation cost is $\$ 548028.191$, and $15.6 \%$ of the demand is served by the local generation units. Therefore, the improvement in the restored demand by PV generation is $32 \%$. The solution time for the 5 -hour operation is 42:22 min and the CPLEX time is $7.14 \mathrm{sec}$.

To accelerate computation speed, the rolling horizon approach is used to solve this problem. The expected operation cost in 6:00-6:15 AM is $\$ 26801.385$ and $18.1 \%$ of the demand is being served in the first operation horizon. The solution time is $2: 53 \mathrm{~min}$ and the CPLEX time is $0.27 \mathrm{sec}$, which is less than 5 minutes.

\section{Simulation on Large-scale test system}

The large-scale system, which is consisted of 3 existing test systems (EPRI ckt5 system, EPRI ckt7 system, IEEE 8500 bus system) is used as a test case. Here, 25 PV units are integrated into the test system. The capacities of PV1, PV2, PV6, and PV7 are $400 \mathrm{~kW}$ and the capacities of other PV units are $200 \mathrm{~kW}$. PV1-PV8 are Type III PV units and coupled with ESS1-ESS8, respectively. PV9-PV12 are Type II PV units and coupled with ESS15-ESS18, respectively. The rest of the PV units are Type I. In this network, 15 DERs with black start capability, are installed and the capacity of each DER is $500 \mathrm{~kW}$. The simulation is performed on a server with Dual 14 Core Intel Xeon $2.6 \mathrm{GHz}$ and $380 \mathrm{~GB}$ RAM with CPLEX 12.9.0. 
Table 3-3 shows the simulation results in case of outages in the main feeder and distribution network with and without PV generation. When PV generation is integrated to facilitate the distribution restoration, $43.8 \%$ of the load is served in the operation horizon. If there is no PV generation in the network, only $35 \%$ of the demand is served by the local generation units. Therefore, the improvement in the restored demand by PV generation is $25.1 \%$.

Figure 3-9 shows the percentage of the restored load over the considered period with and without PV generation. As can be observed from the figure, PV integration could improve the restored load significantly. The solution time for the 5-hour operation is more than 5 minutes. Therefore, we used the rolling horizon approach to solve the problem for 15 minutes with 5 minutes time step and update the results every 5 minutes.

Table 3-3. Simulation results for the large-scale test system with/without PV generation

\begin{tabular}{|c|c|c|c|c|}
\hline & $\begin{array}{c}\text { Served load } \\
\text { (kWh) }\end{array}$ & $\begin{array}{c}\text { Percentage of } \\
\text { served load }\end{array}$ & Cost (\$) & $\begin{array}{c}\text { Solution time } \\
\text { (min) }\end{array}$ \\
\hline With PV generation & $61,410.6$ & $43.8 \%$ & $2,462,985.0$ & 31.44 \\
\hline Without PV generation & $49,072.0$ & $35.0 \%$ & $2,848,189.4$ & 18.05 \\
\hline
\end{tabular}

Using the rolling horizon approach to solve this problem, $35.6 \%$ of the demand is being served in the first operation horizon, i.e., 6:00-6:15 AM. The solution time is $\mathbf{4 2 . 2 5 2} \mathbf{~ s e c}$ and the CPLEX time is $27.73 \mathrm{sec}$, which is far less than 5 minutes.

\section{Task 4 Setting up test cases used for pre-event preparation and post-event operation optimization solution algorithms}

\begin{tabular}{|l|l|}
\hline Tasks & \multicolumn{1}{c|}{ Description } \\
\hline T4 & $\begin{array}{l}\text { Task Name: Setting up test cases used for pre-event preparation and post-event operation } \\
\text { optimization solution algorithms } \\
\text { Task Description: Set up both small-scale and large-scale test cases including distribution } \\
\text { feeder models, damage scenarios, solar energy penetration levels, and other flexible resource } \\
\text { configurations. }\end{array}$ \\
\hline T4.1 & $\begin{array}{l}\text { Set up small-scale test cases with three-phase single feeder systems } \\
\text { Completion in Q1-FY19: Set up small-scale test case based on the IEEE-123 test system. } \\
\text { Prepare extreme weather information and fragility model }\end{array}$ \\
\hline T4.2 & Set up large-scale test cases with three-phase multiple feeder systems \\
\hline
\end{tabular}




\begin{tabular}{|l|l|}
\hline & $\begin{array}{l}\text { Completion in Q2-FY19: Set up a large-scale test case by merging } 3 \text { large-scale test } \\
\text { systems. Demonstrate results of test case generation mechanism framework }\end{array}$ \\
\hline T4.3 & $\begin{array}{l}\text { Data preparation of real feeder data } \\
\text { Completion in Q4-FY19: Set up real feeder test case by preparing NDA with utility partners. } \\
\text { Updated PV placement scenarios and crew dispatch configurations. }\end{array}$ \\
\hline $\begin{array}{l}\text { Deliverables: Small-scale test system adapted from the IEEE-123 test system } \\
\text { Large-scale test system with 14,319 nodes }\end{array}$ \\
$\begin{array}{l}\text { The real feeder test system contains } 240 \text { nodes, } 233 \text { lines, and } 9 \text { switches } \\
\text { Test case generation mechanism }\end{array}$ \\
\hline
\end{tabular}

\section{Subtask 4.1: Set up small-scale test cases with three-phase single feeder systems Small-scale test case}

The small-scale system used in this project is an updated version of the IEEE 123-bus test system. This feeder model operates at a nominal voltage of $4.16 \mathrm{kV}$. It has 129 nodes, 129 lines, 11 switches, 4 regulators, and 1 transformer. In this project, the test system is projected on the map according to its actual size defined by line length.

\section{Test case generation mechanism}

The test case generation mechanism includes 3 major steps: (1) Generate weather metric of extreme weather events; (2) Prepare fragility model of test systems which describes the behavior of components in test system under extreme weather events; (3) Acquire damage status of components in test system subject to specific extreme weather events. Subtask 4.2: Set up large-scale test cases with three-phase multiple feeder systems Large-scale test case

The large-scale system considered in this project is a composite of 3 large-scale test systems that include the EPRI Ckt5 system, EPRI Ckt7 system, and IEEE 8500-bus test system. This merged system has 9,057 buses and 14,319 nodes, which meets the project requirement of over 10,000 nodes. As shown in Figure 4-1, the three individual test systems are interconnected at the substation marked by a red diamond. The substation is assumed to have a transformer that steps down the voltage at the primary side from $115 \mathrm{kV}$ to 12.7

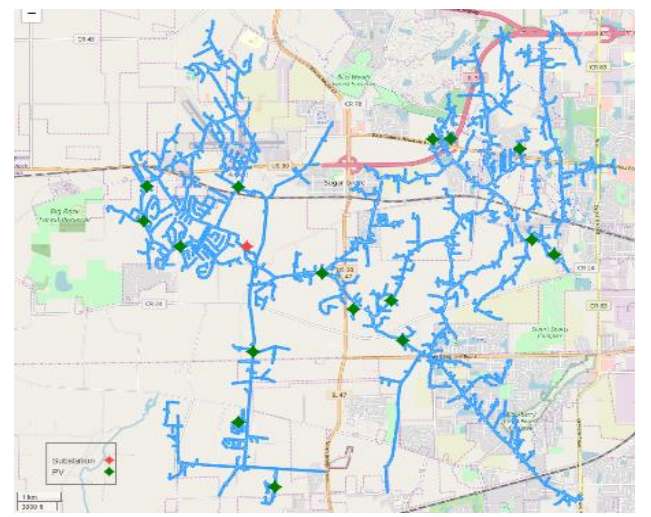

Figure 4-1. Topology of large-scale test system. $\mathrm{kV}$ at the secondary side. Each test system connects to the secondary side of the transformer and spreads to the hypothetical service territory. The topology was changed slightly to avoid overlapping lines. Figure 4-1 shows the geographical location and connectivity of primary buses at $7.2 \mathrm{kV}$. The secondary buses with low-voltage levels are not shown due to a lack of geographical information. While performing the system integration work, the project team has identified several bugs in the interface tool provided by OpenDSS (e.g., mismatch data returned by OpenDSS functions), as well as some 
model errors in the test feeders provided by OpenDSS (e.g., missing buses, duplicated lines).

The damage scenarios are generated by sampling according to the failure probability derived from the weather forecasting and element fragility curve from subtask 4.1. For post-event restoration, we use a single damage scenario in order to compare the resilience improvement of different PV penetrations.

We consider three types of PVs in the large-scale system. The assumptions and definitions are demonstrated in Table B-1. Specific to this large-scale test system, The capacity for Type III, II, and I PVs are $2000 \mathrm{~kW}, 48 \mathrm{~kW}$, and $6 \mathrm{~kW}$ respectively. Table 4-1 below summarizes the number of different types of PV for each scenario.

Table 4-1. Test Scenarios Used for Case Study

\begin{tabular}{|c|c|c|c|c|c|c|c|}
\hline Penetration & Type III PV & Type II PV & Type I PV & Penetration & Type III PV & Type II PV & Type I PV \\
\hline $9 \%$ & 1 & 1 & 8 & $63 \%$ & 7 & 9 & 63 \\
\hline $18 \%$ & 2 & 3 & 16 & $72 \%$ & 8 & 10 & 64 \\
\hline $27 \%$ & 3 & 4 & 24 & $81 \%$ & 9 & 12 & 72 \\
\hline $36 \%$ & 4 & 6 & 32 & $90 \%$ & 10 & 13 & 80 \\
\hline $45 \%$ & 5 & 7 & 40 & $99 \%$ & 11 & 15 & 88 \\
\hline $54 \%$ & 6 & 7 & 48 & $99 \%$ with more Type I PV & 11 & 0 & 208 \\
\hline
\end{tabular}

\section{Results of test case generation mechanism}

Based on the generation mechanism developed in subtask 4.1, the generated test cases include not only the damage status of electric components but also the repair time for the pre-event evaluation in Task 2 and outage scenarios for post-event energy management in Task 3.

For the hurricane extreme weather events, the evolution process of a hurricane is depicted [T4-1]. The hurricane simulations are performed for a period of 24 hours [T4-2], [T4-3]. The wind speed at a distribution line can be represented by a function of the distance from the distribution line to the hurricane eye [T4-4].

For flood extreme weather events, it is pointed out in [T4-5] that the elevation difference determines the potential flood risk, so the flood depth distribution in this project is determined based on the regional elevation and the street configuration [T4-5]-[T4-8]. For the winter storm extreme weather events, it is a combined impact of wind and ice. In this project, the weather metric of wind speed is adapted from a recorded hurricane in [T4-4] and it is observed in [T4-9] - [T4-12] that the ice thickness is under the impact of wind speed, elevation, and icing durations so the weather metric of ice thickness is adapted from a recorded winter storm event in [T4-10]. The results demonstration is similar to previous sections and thus omitted in FTR due to space limitations.

For hurricane extreme weather events, the fragility model is based on the threshold simulation method [T4-13]-[T4-15]. The fragility curves under flood events are adapted from HAZUS software as a piece-wise linear function [T4-16]. We consider three different electric components: poles, substations, and PV panels. The fragility curve under a winter storm follows the lognormal cumulative distribution function [T4-17]. The mean and 
variance parameters of the distribution are obtained from [T4-17] - [T4-19] for the wind impact and 3 different levels of ice thickness (heavy / medium / no icing).

The results focus on the visualization of damage status of both small-scale and largescale test systems subject to three types of extreme weather events: hurricane, flood, and winter storm (omitted in FTR due to space limitations).

1) Test case under the hurricane extreme weather events

Using the evolution mechanism, both small-scale test cases and large-scale test cases have been generated under the hurricane extreme weather events. It should be noted that only wind speed is considered in hurricane extreme weather. The impact of flood depth in the hurricane can be integrated into the current framework in future studies.

The IEEE 123-bus system is fitted into an area that covers the range of latitude $(28.97 \circ \mathrm{N}$ $-29.98 \circ \mathrm{N})$ and longitude $(95.48 \cdot \mathrm{W}-95.47 \circ \mathrm{W})$, which is close to the coastline. It is assumed that hurricanes land at the location with latitude $27.6 \circ \mathrm{N}$ and longitude $97.3 \circ \mathrm{W}$. The hurricanes are assumed to be moving with a translational speed of 12.5 mph and traveling for 24 hours after landfall. Figure 42(a) illustrates the forecasted
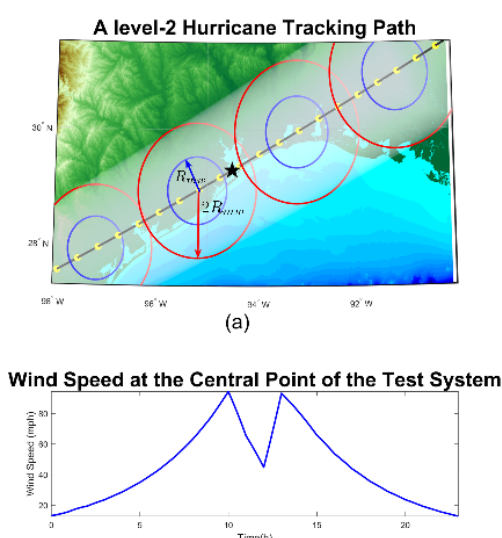

(b)

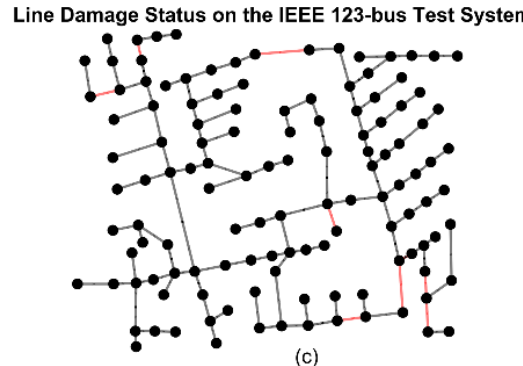

(c)

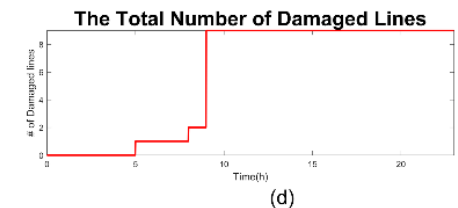

Figure 4-2. Simulating line damage status during a level-2 hurricane track of a category-2 hurricane (with the maximum wind speed at the landfall location), and its time-varying impacts on the test system. The yellow dots represent the locations of the hurricane eye at different times. The black star shows the location of the test system. The blue circle indicates the boundary of the maximum winds for the traveling hurricanes at a certain eye location. The area between the blue circle and the red circle experiences $82.5 \%$ of the maximum wind speed. The wind speed at the center of the test system during a category-4 hurricane is shown in Figure 4-2 (b). Figure 4-2 (c) shows the simulated line damage status on the test system at $t=24$. The accumulated total number of damaged lines at a different time is shown in Figure 4-2 (d).

Although Figure 4-2 is showing the cumulative results at the end of the $24^{\text {th }}$ hour, each hour in this process can be separately illustrated to showcase the evolution process during hurricane extreme weather events. We also generated test cases under category3 and category-4 hurricanes. We also projected the large-scale system composite with over 10,000 nodes into an area that covers the range of latitude $(29.44 \circ \mathrm{N}-29.56 \circ \mathrm{N})$ and longitude $(95.46 \circ \mathrm{W}-95.5 \circ \mathrm{W})$. The test cases of the large-scale system under 3 different categories are omitted in FTR due to space limitations. 
2) Test case under flood extreme weather events

Different from hurricane events, test cases under flood events use the snapshot mechanism and we also utilized a different visualization method to show this process.

The major steps in the snapshot mechanism have all been depicted in the layers on visualization. For example, the weather metric generation is depicted as a "flood depth" layer on top of the map layer; the "failure probability" layer showcase the failure probability of electric components according to the fragility curves under the flood events. As shown in Figure 4-3, the "damage status" layer showcase

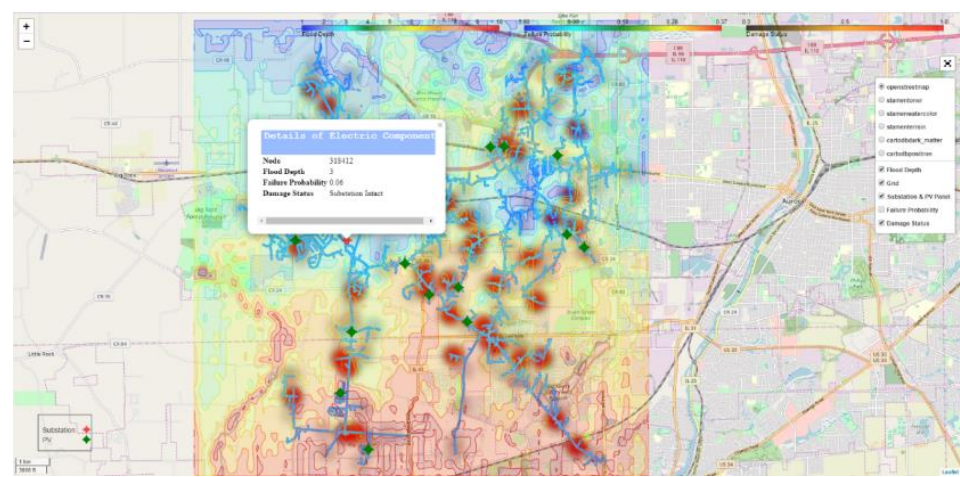

Figure 4-3. Demonstration example of test case visualization. the electric component is damaged. We can also click on all 3 types of electric components to view the details such as topology, weather metric, failure probability, and damage status.

Based on the above-mentioned snapshot mechanism and visualization method, test cases have been generated for both small-scale and large-scale systems under flood extreme weather events.

The flood depth distribution is determined by the elevation of the selected region, which is the west suburbs of Chicago. The visualization of the damage status of the large-scale system is shown in the demonstration example of Figure 4-3 with the weather metric layer showing the flood depth distribution. 139 out of 3673 lines are damaged in this test case and the 16 PV on average is generating at $83 \%$ of original capacity.

\section{Subtask 4.3: Data preparation of real feeder data}

The project team developed a set of test scenarios using the real feeder data collected in Task 6. As shown in Figure 4-4, the real feeder system is located in the Midwest U.S. It consists of 3 feeders and contains 240 nodes, 233 lines, and 9 switches ( 3 are normally open). The system has standard electric components such as overhead lines, underground cables, substation transformers with LTC, line

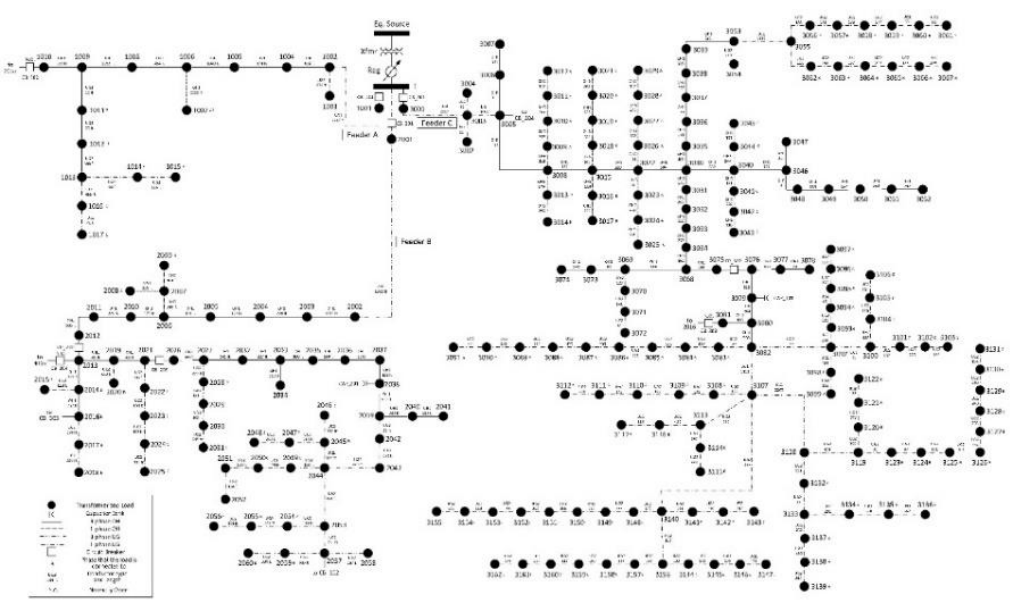

Figure 4-4. One-line diagram of the distribution system. 
switches, capacitor banks, and secondary distribution transformers. Details of this real feeder system can be obtained at [T4-20].

In addition, 4 crew depots are hosting 18 crews. The depots are also in charge of dispatching 1 DERs, 4 mobile DERs, and 3 mobile energy storage. These resources, including crew and power sources, are assumed to be dispatched to the field and connected properly to the distribution feeders before the weather event. The allocation solution will be generated by the algorithm developed by ISU.

The damage scenarios are generated by sampling according to the failure probability derived from the wind speed of hurricane extreme weather and element fragility curve. For post-event restoration, we use a single damage scenario to compare the resilience improvement of different PV penetrations.

To investigate the benefits of PV at different penetrations, we have developed a set of test scenarios by allocating different numbers and different types of PVs in the system. The assumptions and definitions of PV types are demonstrated in Table B-1. Specific to this real feeder system, The capacity for Type III, II, and I PVs are 600kW, 12kW, and $5 \mathrm{~kW}$ respectively.

\begin{tabular}{|c|c|c|c|c|}
\hline \multicolumn{2}{|c|}{ Table 4-2. Test Scenarios Used for Case Study } \\
\hline PV Penetration Level & Type III Number & Type II Number & Type I Number & Residential PV Percentage (\%) \\
\hline $\mathbf{0} \%$ & 0 & 0 & 0 & N/A \\
\hline $\mathbf{1 0} \%$ & 0 & 7 & 6 & 26.32 \\
\hline $\mathbf{2 0} \%$ & 0 & 9 & 25 & 53.65 \\
\hline $\mathbf{3 0} \%$ & 0 & 9 & 49 & 69.41 \\
\hline $\mathbf{4 0} \%$ & 0 & 9 & 72 & 0 \\
\hline $\mathbf{5 0} \%$ & 1 & 0 & 0 & 4.20 \\
\hline $\mathbf{7 0} \%$ & 1 & 7 & 6 & 15.01 \\
\hline $\mathbf{8 0} \%$ & 1 & 9 & 25 & 25.71 \\
\hline $\mathbf{9 0} \%$ & 1 & 9 & 49 & 33.71 \\
\hline
\end{tabular}

Table 4-2 summarized the number of different types of PV for each scenario. It should be noted that there are 2 "turning points" in the residential PV percentage compared with the increase of PV penetration level. 1) From $40 \%$ to $50 \%$ of penetration, all Type II and Type I PV are merged to form one single Type III PV, which is a central large utility PV farm. This "turning point" (highlighted in blue) is designed to test the performance of centralized PV versus distributed PV. 2) From $90 \%$ to $100 \%$ penetration, all Type II PV are merged to place more Type I PV in the system. This "turning point" is designed to test the system performance when there is a large number of residential, Type I PV in the system. In addition, between these two turning points, the increasing pattern of Type II and Type I is much similar (0\%-40\% and 50\%-90\%).

In addition, for all the PV penetration levels, we also prepared a test scenario under which the pre-event preparation results are not utilized, this is referred to as the "base model" in the following sections. These scenarios are designed to verify the resilience improvement benefit by incorporating pre-event preparations. 


\section{Task 5 Extensive case studies to evaluate the benefits of solar energy in} resilience improvement

\begin{tabular}{|l|l|}
\hline Tasks & $\begin{array}{l}\text { Task Name: Extensive case studies to evaluate the benefits of solar energy in resilience } \\
\text { improvement } \\
\text { Task Description: Conduct case studies to quantify resilience improvement by solar energy } \\
\text { at various penetration levels, coordination with other flexible resources, and interaction of pre- } \\
\text { event and post-event optimization. }\end{array}$ \\
\hline T5.1 & $\begin{array}{l}\text { Conduct extensive case studies based on large-scale test cases to evaluate the resilience } \\
\text { benefits of solar energy at different penetration levels and coordination of solar energy with } \\
\text { other flexible resources. The impact of the resource availability from the pre-event preparation } \\
\text { to the post-event operation will be evaluated via sensitivity analysis. The impact of } \\
\text { coordination between pre-event preparation optimization and post-event operation } \\
\text { optimization will be assessed in the case studies. } \\
\text { Completion in Q2-FY20: Conducted extensive cases on a large-scale test system to evaluate } \\
\text { the resilience benefit of solar energy. Such as the benefit coming from coordination between } \\
\text { pre-event preparation and post-event operation is demonstrated through extensive case } \\
\text { studies. In addition, we further verified this using real feeder system data. }\end{array}$ \\
\hline $\mathbf{M}$ & $\begin{array}{l}\text { Case studies on the evaluation of benefits of solar energy and its coordination with other } \\
\text { flexible resources in grid resilience improvement; the impact of coordination between pre- } \\
\text { event and post-event optimization } \\
\text { 100\% Completion: Complete extensive case studies with the conclusion that total resilience } \\
\text { improvement increases with the increase of PV penetration level, while the marginal benefit of } \\
\text { PV diminishes when the PV penetration level continues to increase. } \\
\text { Verified the benefit of coordination between pre-event preparations and post-event operations }\end{array}$ \\
\hline Deliverables: Additional extensive case studies as verification
\end{tabular}

\section{Subtask 5.1: Extensive case studies on a large-scale test system}

In order to evaluate the performance of the developed model, the model is compared to a typical utility approach to disaster preparation. The base case is generated by following the procedure below:

1. Mobile generators are prepositioned at

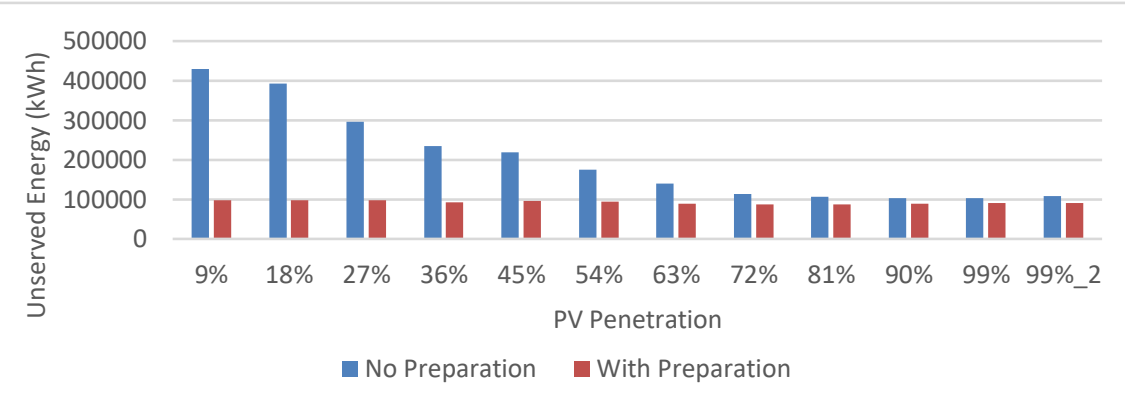

Figure 5-1. Comparison on large-scale test case. the substations.

2. Extra mobile generators are prepositioned at high-priority loads.

3. PV and battery storage are not considered

4. Fuel is allocated to the mobile generators such that they can operate for at least 24 hours. 
5. Crews are allocated evenly between depots. In case of an odd number of crews, the location with more components will have a higher number of crews.

As shown in Figure 5-1, the case studies on large-scale test cases under hurricane extreme weather demonstrate that with coordination in the proposed model, the unserved energy is significantly lower than that of the base model without coordination.

To further verify this benefit, we conducted a similar test on the real feeder system. The detailed results are omitted in FTR due to space limitations. However, both indexes in the resilience improvement of the proposed model are significantly higher than the base model without coordination between pre-event preparations and post-event operations. In summary, in addition to the large-scale test system, the benefit of proper pre-event preparation can help on post-event restoration is verified on the real feeder system.

\section{Task 6 Testing of the pre-event and post-event optimization via simulation using real feeder data}

\begin{tabular}{|c|c|}
\hline Tasks & Description \\
\hline T6 & $\begin{array}{l}\text { Task Name: Testing of the pre-event and post-event optimization via simulation using real } \\
\text { feeder data } \\
\text { Task Description: Perform testing of the models and solution algorithms of pre-event and } \\
\text { post-event optimization using real test feeder data provided by the utility partners. }\end{array}$ \\
\hline T6.1 & $\begin{array}{l}\text { Continue real feeder data preparation for the testing. } \\
\text { Completion in Q1-FY20: Collaborated with partner utilities to prepare the feeder data from } \\
\text { their distribution system. }\end{array}$ \\
\hline $\begin{array}{l}M \\
2.6 .1\end{array}$ & $\begin{array}{l}\text { Data interface development in software platforms (e.g., Matlab or Python) for the real feeder } \\
\text { data provided by utility partners. } \\
100 \% \text { Completion: A set of MATLAB-based data interfaces are developed from importing } \\
\text { read feeder data and aligning the solutions among the team }\end{array}$ \\
\hline T6.2 & $\begin{array}{l}\text { Testing of the pre-event preparation optimization model and solution algorithms via simulation } \\
\text { using real feeder data provided by utility partners (e.g., City of Bloomfield utility, Algona } \\
\text { Municipal Utilities). } \\
\text { Completion in Q2-FY20: Completed extensive case studies of pre-event preparations } \\
\text { optimization on real feeder test systems }\end{array}$ \\
\hline $\begin{array}{l}M \\
2.6 .2\end{array}$ & $\begin{array}{l}\text { Case studies of pre-event preparation optimization under real feeder data within required } \\
\text { computation time (e.g., } 4 \text { hours) completed and results being reviewed by the utility; the } \\
\text { resilience improvement will be at least } 10 \% \text { in terms of served energy and outage duration } \\
\text { reduction } \\
100 \% \text { Completion: Complete the extensive case studies on the real feeder system } \\
\text { considering different PV penetrations. The computation time is } 2.5 \text { hours with around } 17 \% / \\
25 \% \text { resilience improvement in served energy and reduction of outage duration respectively }\end{array}$ \\
\hline T6.3 & $\begin{array}{l}\text { Testing of the post-event operation optimization model and solution algorithms via simulation } \\
\text { using real feeder data provided by utility partners (e.g., City of Bloomfield utility, Algona } \\
\text { Municipal Utilities). } \\
\text { Completion in Q2-FY20: Completed extensive case studies of post-event operation } \\
\text { optimization on real feeder test systems }\end{array}$ \\
\hline $\begin{array}{l}M \\
2.6 .3\end{array}$ & $\begin{array}{l}\text { Case studies of post-event operation optimization under real feeder data within required } \\
\text { computation time ( } 5 \text { min for energy management optimization and } 1 \text { hour for restoration }\end{array}$ \\
\hline
\end{tabular}


optimization) completed and results being reviewed by the utility; the resilience improvement will be at least $10 \%$ in terms of served energy and outage duration reduction

$100 \%$ Completion: Complete the extensive case studies on the real feeder system considering different PV penetrations. The computation time is less than 1 hour with resilience improvement ranging from $23 \%$ to $45 \%$

Deliverables: Data interface to unify pre-event and post-event optimization solutions Real feeder test case using developed pre-event preparation and post-event operation framework

\section{Subtask 6.1: Continue real feeder data preparation for the testing.}

The project team collaborated with partner utilities to prepare the feeder data from their distribution system. Details on the real system can be found in the section of subtask 4.3. As shown in Figure 6-1, the ANL team has developed a data interface to import system models from the OpenDSS data file. The data interface was implemented in MATLAB by leveraging the COM interface provided by OpenDSS, which indicates $100 \%$ completion of Milestone 2.6.1. The interface was designed to be able to import any OpenDSS system model information. The PV, DER, and crew information is organized in Excel format and imported into MATLAB through the functions provided by MATLAB. In addition, to ensure seamless and efficient coordination among the team, the ANL team also developed the data interfaces to import the pre-event preparation solutions provided by ISU, and reformat the post-event restoration solution provided for SMU.

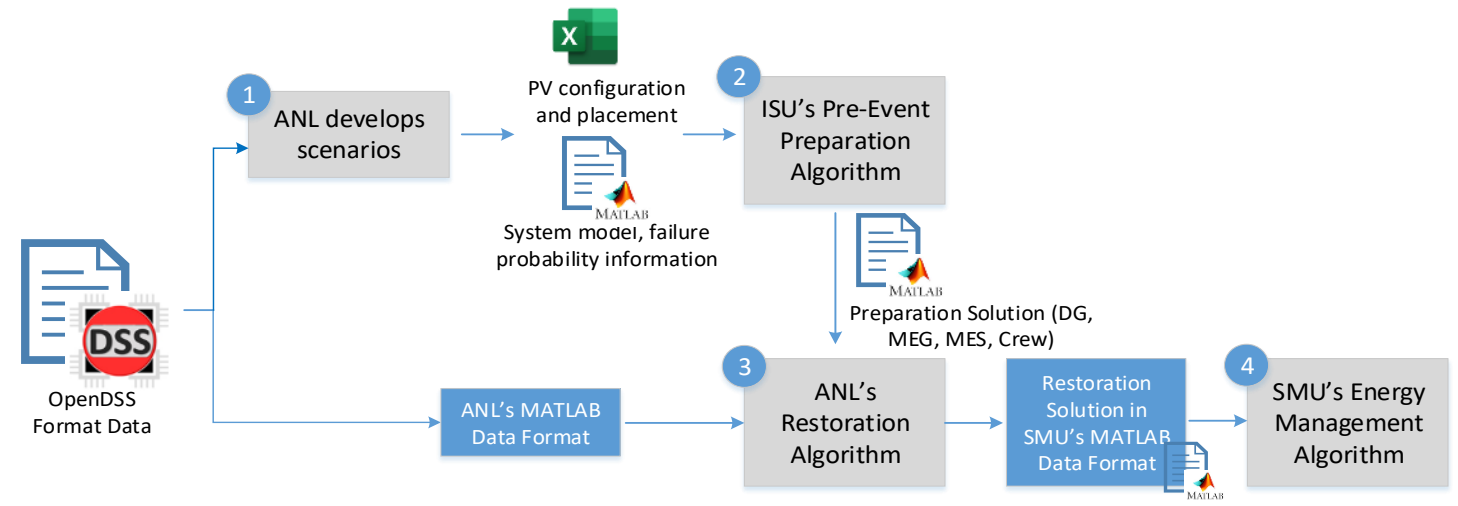

Figure 6-1. Data interface

\section{Subtask 6.2: Testing of the pre-event preparation optimization model and solution algorithms via simulation using a real feeder system.}

To show the advantages of the PV systems, the project team generates a random scenario and tests the response of the system with the proposed method and varying PV penetration levels. The generated scenario has 15 damaged lines and we assume the substation is not receiving power from the transmission system for 6 hours.

In this report, we compare the pre-event preparation results with various levels of PV penetrations demonstrated in Table B-1 and Table 4-2. The pre-event models with various PV penetration levels and 10 damage scenarios are solved. The first-stage decision variables (locations of mobile energy generator (MEG), mobile energy storage Page $\mathbf{4 0}$ of 56 
(MES), and crews) with different PV penetration levels are different. For all the base models without pre-event preparations under various PV penetration, they would follow the same intuitive dispatch as depicted in Figure 62.

Figure 6-3 shows the percentage of power served during the event, and after the repair process starts. Table 6-1 compares the amount of load served and average outage duration with different levels of PV penetration for the proposed model with pre-event preparations

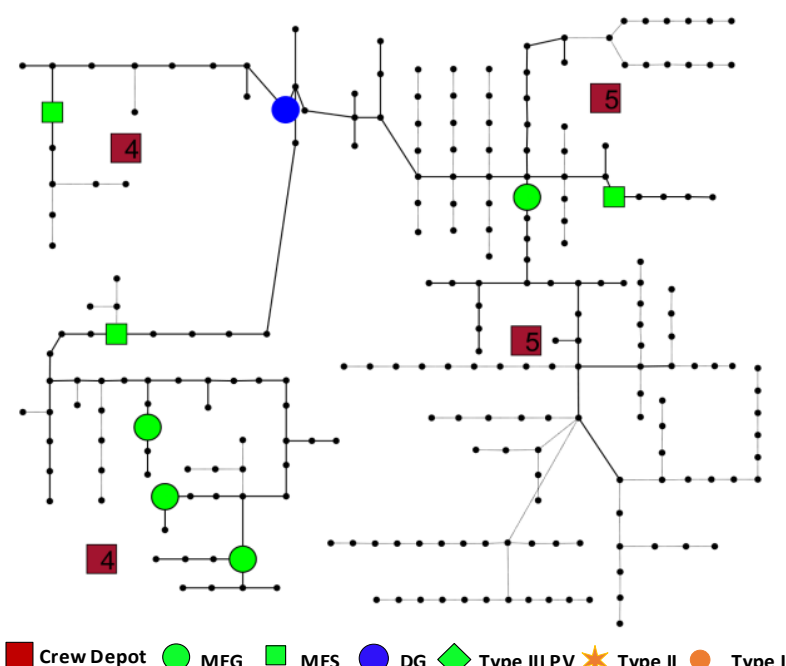

Figure 6-2. Resource allocation in the base model.

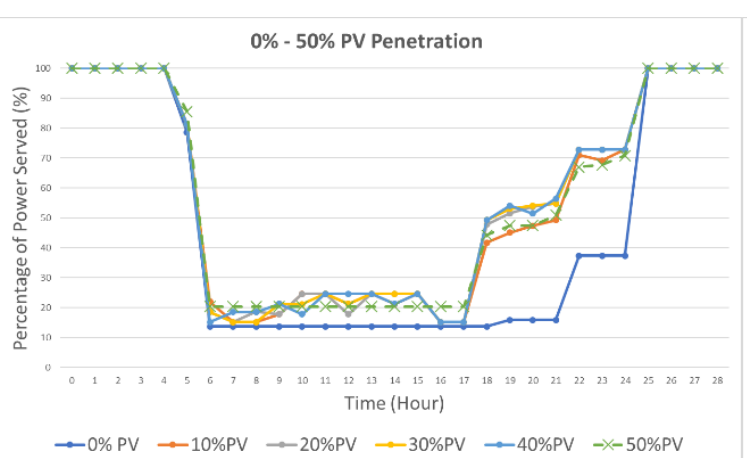

(a)Proposed model 0\%-50\% penetration

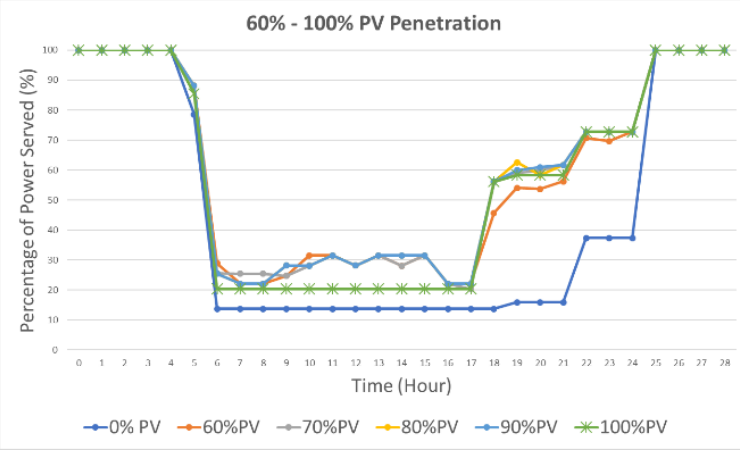

(b) Proposed model 60\%-100\% penetration

Figure 6-3. Load served percentage comparison of the proposed model with various PV penetration levels and base model solution.

Table 6-1. The amount of load served and average outage duration with different levels of PV penetration

\begin{tabular}{|c|c|c|c|c|}
\hline $\begin{array}{c}\text { PV Penetration } \\
\text { Level }\end{array}$ & $\begin{array}{c}\text { Load Energy Served } \\
\text { (kWh) }\end{array}$ & $\begin{array}{c}\text { Resilience } \\
\text { Improvement (\%) }\end{array}$ & $\begin{array}{c}\text { Average Outage } \\
\text { Duration (h) }\end{array}$ & $\begin{array}{c}\text { Resilience } \\
\text { Improvement (\%) }\end{array}$ \\
\hline $\mathbf{0 \%}$ & 10891.0827 & & 15.81122449 & \\
\hline $\mathbf{1 0} \%$ & 13968.8007 & $22.03 \%$ & 13.4744898 & $14.78 \%$ \\
\hline $\mathbf{2 0} \%$ & 14292.4097 & $23.80 \%$ & 13.15816327 & $16.78 \%$ \\
\hline $\mathbf{3 0} \%$ & 14333.5634 & $24.02 \%$ & 13.12244898 & $17.01 \%$ \\
\hline $\mathbf{4 0} \%$ & 14329.59736 & $24.00 \%$ & 13.12244904 & $17.01 \%$ \\
\hline $\mathbf{5 0} \%$ & 14023.9017 & $22.34 \%$ & 13.70918367 & $13.29 \%$ \\
\hline $\mathbf{6 0} \%$ & 15228.621 & $28.48 \%$ & 13.33673469 & $15.65 \%$ \\
\hline $\mathbf{7 0} \%$ & 15551.5442 & $29.97 \%$ & 13.08163265 & $17.26 \%$ \\
\hline $\mathbf{8 0} \%$ & 15607.2284 & $30.22 \%$ & 13.03571429 & $17.55 \%$ \\
\hline $\mathbf{9 0} \%$ & 15607.2284 & $30.22 \%$ & 13.03571429 & $17.55 \%$ \\
\hline $\mathbf{1 0 0} \%$ & 14589.4421 & $25.35 \%$ & 13.21428571 & $16.42 \%$ \\
\hline
\end{tabular}


It can be seen that the penetration of PV contributes to enhancing system resilience. Approximately $30.22 \%$ more loads are served compared to the base model with $90 \% \mathrm{PV}$ penetration. Also, the average outage duration decreased by $17.55 \%$.

However, additional observations are obtained for the test system at the abovementioned two "turning points" (two rows highlighted in blue). 1) From $40 \%$ to $50 \%$ penetration level when all distributed PVs are merged as a centralized large PV farm, the resilience improvement decreased, which demonstrates the importance and benefit of distributed PV in resilience improvement. 2) From $90 \%$ to $100 \%$ penetration level when all distributed PVs are converted to small residential PVs, meaning there is a significant amount of residential PVs without dispatch-ability in the system, it is observed that the resilience improvement decreased, which demonstrate the importance and necessity of dispatch-ability under high PV penetration scenarios.

Subtask 6.3: Testing of the post-event operation optimization model and solution algorithms via simulation using a real feeder system.

In subtask 6.3, extensive case studies on a real test feeder are performed to evaluate the benefits of solar energy and coordination between pre-event preparation and post-event operation. To verify the proposed post-event energy management model is applicable for the long-duration operation, a 5-day operation simulation is performed that uses a twotime-scale operation strategy.

In the large time-scale simulation, the time step and operation horizon are chosen as 15 minutes and 5 days, respectively. In the small time-scale simulation, these are selected as 5 minutes and 15 minutes. As the large time-scale simulation considers the entire operation horizon, the short-sightedness associated with limited information for the complete 5-day operation is avoided. Here, the procured state of charges for ESS in the large time-scale simulation provides a reference for the short time-scale simulation.

\section{Post-event Energy Management}

1) Evaluating the benefits of PV generation and coordination between pre-event and post-event restoration efforts

To evaluate the benefits of solar energy, 11 cases are considered with different PV penetration levels (0\%-100\%). To address the benefits of coordination, these 11 cases are considered as coordinated cases (Case 1- Case 11) and base cases (Case 1b-Case 11b). The coordinated cases are benefited from the coordination between the pre-event preparation and post-event operation while the bases cases do not consider any coordination between the pre-event preparation and post-event operation. The simulation results of the coordinated cases are compared with those of the base cases to validate the effectiveness of the coordination. The basic definition of PV scenarios is illustrated in Table B-1. The capacities of Type I PV, Type II PV, and Type III PV units are $5 \mathrm{~kW}, 12$ $\mathrm{kW}$, and $600 \mathrm{~kW}$, respectively. Each Type II PV unit is coupled with an energy storage system with $12 \mathrm{~kW}$ power capacity and $96 \mathrm{kWh}$ energy capacity. Each Type III PV unit is coupled with an ESS of $600 \mathrm{~kW} / 4,800 \mathrm{kWh}$ capacity. For each case, 2 fixed DERs, 4 mobile DERs, and 3 mobile ESSs are integrated, and 15 areas are damaged. The main 
feeder is also out of service. The capacity of each fixed DER and mobile DER is $300 \mathrm{~kW}$. The power capacity and energy capacity of each mobile ESS are $300 \mathrm{~kW}$ and 2,400 kWh, respectively.

Table 6-2 Simulation results for coordinated cases and base cases with different PV penetration levels

\begin{tabular}{|c|c|c|c|c|c|c|c|c|}
\hline Case & $\begin{array}{l}\text { Percentage } \\
\text { of unserved } \\
\text { load } \%\end{array}$ & $\begin{array}{l}\text { Total } \\
\text { Unserved } \\
\text { energy } \\
\text { (kWh) }\end{array}$ & $\begin{array}{c}\text { Percentage } \\
\text { of unserved } \\
\text { critical load } \\
\%\end{array}$ & $\begin{array}{l}\text { Unserved } \\
\text { energy for } \\
\text { the critical } \\
\text { load (kWh) }\end{array}$ & $\begin{array}{l}\text { Percentage } \\
\text { of unserved } \\
\text { noncritical } \\
\text { load } \%\end{array}$ & $\begin{array}{l}\text { Unserved } \\
\text { energy for the } \\
\text { noncritical } \\
\text { load (kWh) }\end{array}$ & $\begin{array}{c}\text { Improvement } \\
\text { of resilience } \\
\%\end{array}$ & $\begin{array}{c}\text { Solution } \\
\text { time } \\
\text { (min) }\end{array}$ \\
\hline Case 1 & 43.60 & $5,169.55$ & 46.68 & $2,450.39$ & 41.16 & $2,719.17$ & 0 & 7.38 \\
\hline Case 1b & 43.60 & $5,169.55$ & 46.68 & $2,450.39$ & 41.16 & $2,719.17$ & - & 7.38 \\
\hline Case 2 & 30.45 & $3,610.05$ & 21.52 & $1,129.52$ & 37.55 & $2,480.53$ & 23.3 & 7.20 \\
\hline Case 2b & 43.20 & $5,121.79$ & 46.14 & $2,422.03$ & 40.86 & $2,699.76$ & - & 7.37 \\
\hline Case 3 & 27.30 & $3,236.65$ & 23.22 & $1,218.99$ & 30.54 & $2,017.66$ & 28.9 & 7.46 \\
\hline Case 3b & 42.93 & $5,089.77$ & 46.04 & $2,417.87$ & 40.44 & $2,671.90$ & - & 7.28 \\
\hline Case 4 & 19.63 & $2,327.38$ & 18.64 & 978.59 & 20.41 & $1,348.79$ & 42.5 & 7.78 \\
\hline Case $4 b$ & 42.90 & $5,086.73$ & 46.00 & $2,414.83$ & 40.44 & $2,671.90$ & - & 7.50 \\
\hline Case 5 & 19.33 & $2,291.94$ & 18.04 & 947.17 & 20.35 & $1,344.77$ & 43.0 & 7.33 \\
\hline Case 5b & 45.93 & $5,445.93$ & 48.89 & $2,566.16$ & 43.59 & 2,879.77 & - & 7.63 \\
\hline Case 6 & 24.61 & $2,918.43$ & 17.84 & 936.28 & 30.00 & $1,982.15$ & 33.7 & 7.42 \\
\hline Case 6b & 49.99 & $5,927.41$ & 53.98 & $2,833.66$ & 46.83 & $3,093.75$ & - & 6.93 \\
\hline Case 7 & 22.88 & $2,712.87$ & 17.83 & 936.12 & 26.89 & $1,776.75$ & 36.7 & 7.53 \\
\hline Case 7b & 48.96 & $5,804.79$ & 53.92 & $2,830.55$ & 45.02 & $2,974.24$ & - & 7.05 \\
\hline Case 8 & 19.51 & $2,312.59$ & 17.78 & 933.62 & 20.87 & $1,378.97$ & 42.7 & 7.62 \\
\hline Case $8 b$ & 48.78 & $5,782.88$ & 53.84 & 2,826.39 & 44.75 & $2,956.49$ & - & 7.33 \\
\hline Case 9 & 18.51 & $2,194.60$ & 17.07 & 896.02 & 19.65 & $1,298.58$ & 44.5 & 7.78 \\
\hline Case $9 b$ & 48.75 & $5,779.84$ & 53.78 & 2,823.35 & 44.75 & $2,956.49$ & - & 7.56 \\
\hline Case 10 & 18.62 & $2,208.21$ & 17.07 & 896.02 & 19.86 & $1,312.19$ & 44.3 & 8.06 \\
\hline $\begin{array}{l}\text { Case } \\
10 b\end{array}$ & 44.90 & $5,840.58$ & 49.22 & $2,834.63$ & 41.47 & $3,005.95$ & - & 8.40 \\
\hline Case 11 & 18.13 & $2,149.12$ & 18.69 & 981.12 & 17.68 & $1,168.00$ & 45.2 & 8.13 \\
\hline $\begin{array}{c}\text { Case } \\
\text { 11b }\end{array}$ & 49.18 & $5,831.07$ & 53.70 & $2,818.98$ & 45.59 & $3,012.09$ & - & 7.76 \\
\hline
\end{tabular}

Table 6-2 summarizes the simulation results for the 11 coordinated cases and 11 base cases. The $3^{\text {rd }}$ and $4^{\text {th }}$ columns list the percentage of unserved load and total unserved energy, respectively. It is observed that both the percentage of unserved load and total unserved energy in Case 1 - Case 11 are lower than those in Case 1b - Case 11b, which validates the effectiveness of the coordination between pre-event preparation and postevent operation. Moreover, we can observe that the total unserved energy decreases with the increase in PV penetration level in Cases 1-11, except for Case 5. The $5^{\text {th }}$ and $6^{\text {th }}$ columns in Table 6-2 demonstrate the percentage of unserved critical load and noncritical load, respectively. In each coordinated case (Case 1 - Case 11), the percentage of the unserved critical load is lower than the percentage of the unserved noncritical load. This validates the higher priority of service restoration for the critical loads. The $7^{\text {th }}$ column shows the improvement of resilience in terms of the served energy for Case 1-11. As shown in this table, when PV units are integrated, the resilience is improved by more than $10 \%$. The last column shows the total solution time for different cases where the overall operation horizon is 10 hours. The solution time for the optimization problem with 
a 15-minute operation horizon is approximately 4 seconds which is far below the simulation time step (5 minutes).

Figure 6-4 shows the total unserved energy as well as the percentage of unserved energy for the coordinated cases (Case 1 - Case 11) and base cases (Case 1bCase 11b) with different PV penetration levels. It is observed that the total unserved energy in Case 1 Case 11 is much smaller than that for the corresponding base cases

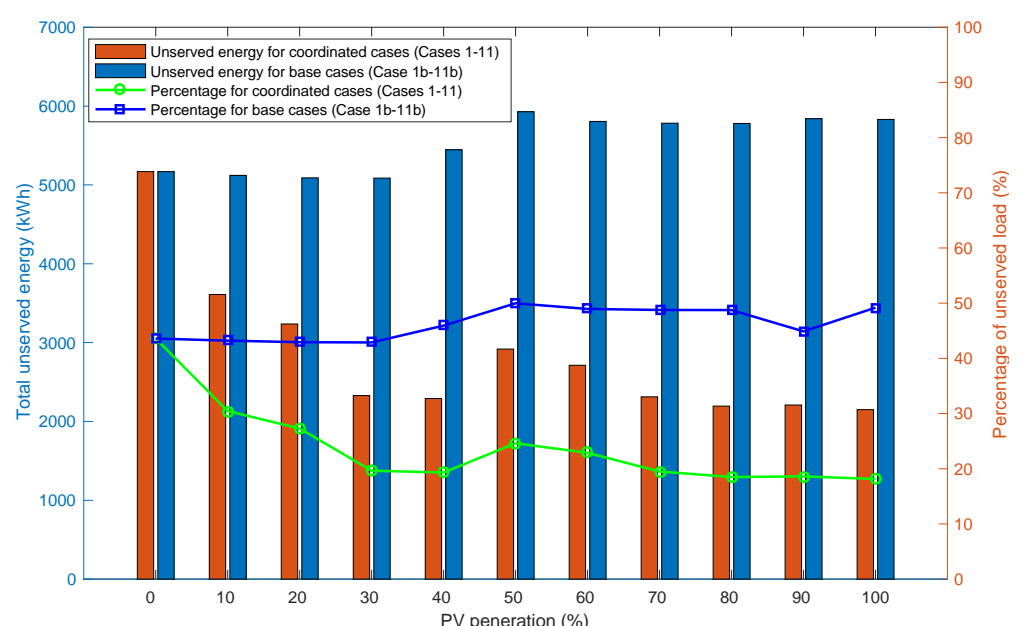

Figure 6-4. Total unserved energy and percentage of unserved energy for the coordinated cases and base cases with different PV penetration level

(Case 1b-Case 11b). Moreover, the PV penetration level has little effect on the total unserved energy and its percentage for Cases 1b-11b. In contrast, for the coordinated cases (Cases 1-11), both the total unserved energy and its percentage decrease substantially with the improvement in PV penetration level from $0 \%$ to $40 \%$. Although the total unserved energy and its percentage increase with the increase in the PV penetration level from $40 \%$ to $50 \%$ due to the decrease in the total number of PV units, they continue to decline as the PV penetration level grows from $50 \%$ to $100 \%$. In the meantime, as the PV penetration level increases, its impact on the reduction of total unserved energy diminishes.

It can be observed in the comparison of unserved load percentage between critical and non-critical loads that the coordination between pre-event preparation and post-event operation leads to a significant reduction of unserved energy for both critical and noncritical loads. Moreover, since the unserved critical loads are maintained at a low level in Cases 1-11, the increase in PV penetration level has little effect on them. In contrast, the unserved noncritical loads in these cases decline sharply first and then smoothly as the PV penetration level increases.

\section{2) Post-event restoration with a 5-day operation horizon}

To apply the proposed post-event operation model to the longer operation horizon, a case study with a 5-day continuous operation is presented. In this section, except for the DER capacity, all other setups are similar to those in Case 6 in Table 6-2.

The total unserved load for 5 days is $26,675.8 \mathrm{kWh}$. The total unserved critical and noncritical loads are $1,022.7 \mathrm{kWh}$ and $25,653.1 \mathrm{kWh}$, respectively. The total solution time is 106.42 minutes. However, the solution time for each 15-minute small time-scale 
simulation using the rolling horizon approach is approximately 4.4 seconds that is far below the simulation time-step (5 minutes). Fig. 6-5 depicts the percentage of the total served load, the total served critical load and the total served non-critical load. It is shown that after the damages are repaired, all critical loads are picked up, while some noncritical

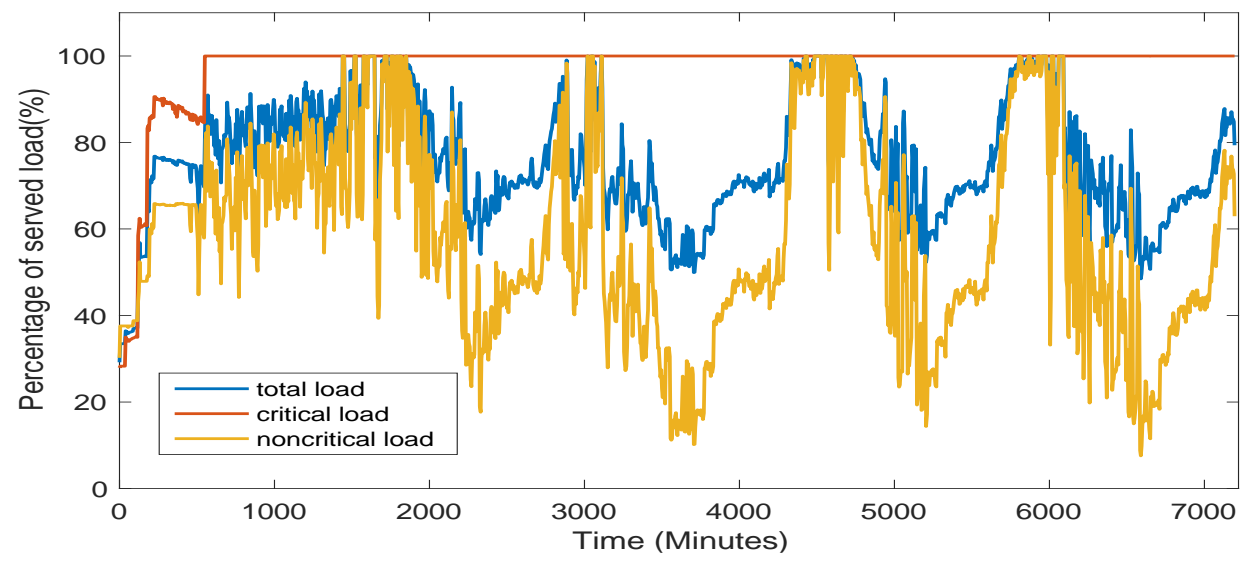

Figure 6-5. Percentage profiles of total served load, total served critical/non-critical loads for the 5-day operation with $60 \%$ PV penetration

loads are curtailed when the total local generation capacity is less than the demand.

Figure 6-6 shows the output of a Type I PV unit for the 5-day operation. It can be seen from this figure that the output of the PV unit changes with solar irradiance.

Figure 6-7 demonstrates the output of a Type II PV unit. Here, the generation by the PV unit follows the solar irradiance pattern similar to the Type I PV unit; however, its coupled ESS charges during the day and discharges at the night to ensure the generation is sufficient to serve the critical loads. Figure 6-8 shows the output of a Type III PV unit. Similar to the Type II PV unit, during the day, the PV unit supplies power mainly by using the PV unit and at night, by using the coupled ESS.

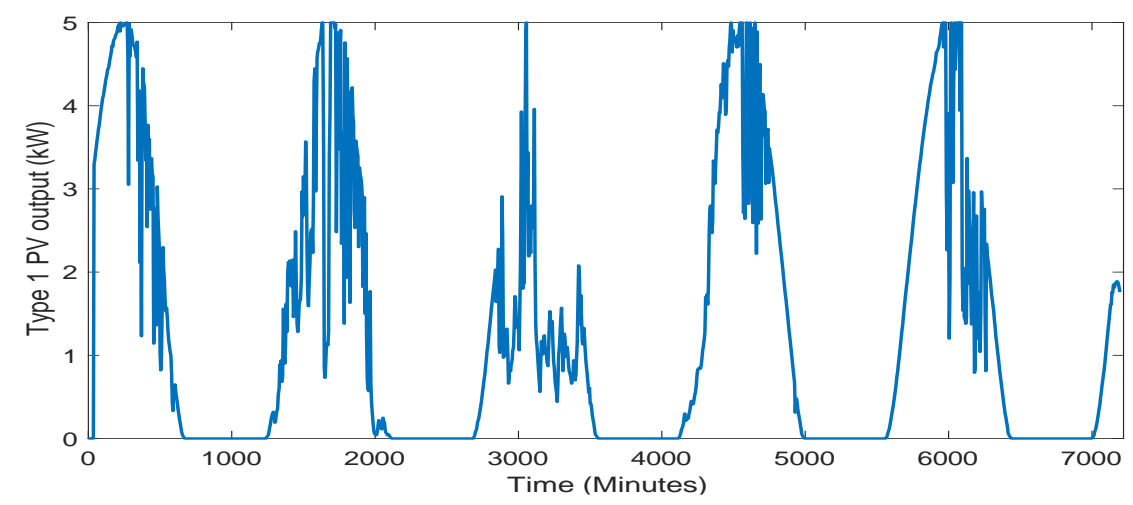

Figure 6-6. PV output profile of a Type-1 PV for the 5-day operation

Page $\mathbf{4 5}$ of 56 


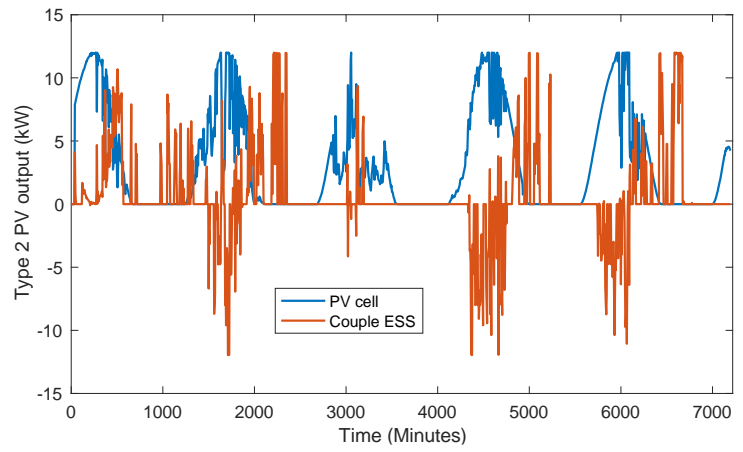

Figure 6-7. PV output profile of a Type-2 PV for the 5-day operation

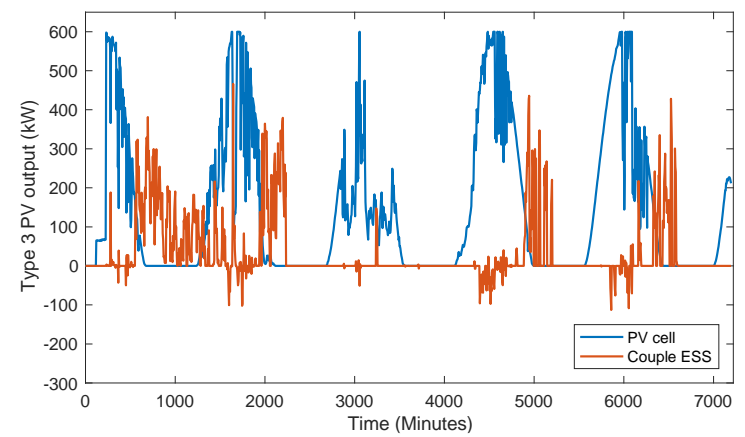

Figure 6-8. PV output profile of a Type-3 PV for the 5-day operation

\section{Post-event Restoration}

Due to the space limit, we present the solution of a sample scenario with PV penetration being $60 \%$. Figure 6-9(a) shows the Percentage of total restored load along time during restoration. The light green lines represent the switch operations at a specific time. It can be observed that at the beginning of the restoration, there is around $10 \%$ of the load served by DERs, MEG, MES, and Type III PVs, which can operate in grid-forming mode and pick up the loads immediately. The switching operations observed at the beginning represent the initial stage of restoration - reconfiguring the system to pick up the loads that are not affected by the damaged components. The rest of the restoration is an integrated process of switch operation and repair. As crews are dispatched to repair the damaged components, the associated loads will no longer be affected and can be picked up, following an optimal order determined by the restoration algorithm.

Figure 6-9(b) shows the energization sequence for the electric power network. Each circle represents a DER with black start capability. The arrows coming out of the circle represent the energization currents. Each black dot represents a block. It can be observed that the system was fully energized and divided into multiple islands.

Figure 6-10(a) shows the single-line diagram of the energized test system supported by all the power sources. The system was partitioned into multiple islands that are labeled by different colors. Note that each island contains one power source with black start capability and multiple switches and power sources without black start capability. Figure 6-10(b) shows the dispatch sequence for repair crews. The dispatch sequence is mapped on the figure according to the coordinates of damaged components. Each circle represents a depot that is hosting the crews. The crews come out of different depots and are labeled in different colors. The total number of crews dispatched out of a depot is limited by the pre-event preparation solution provided by ISU. 

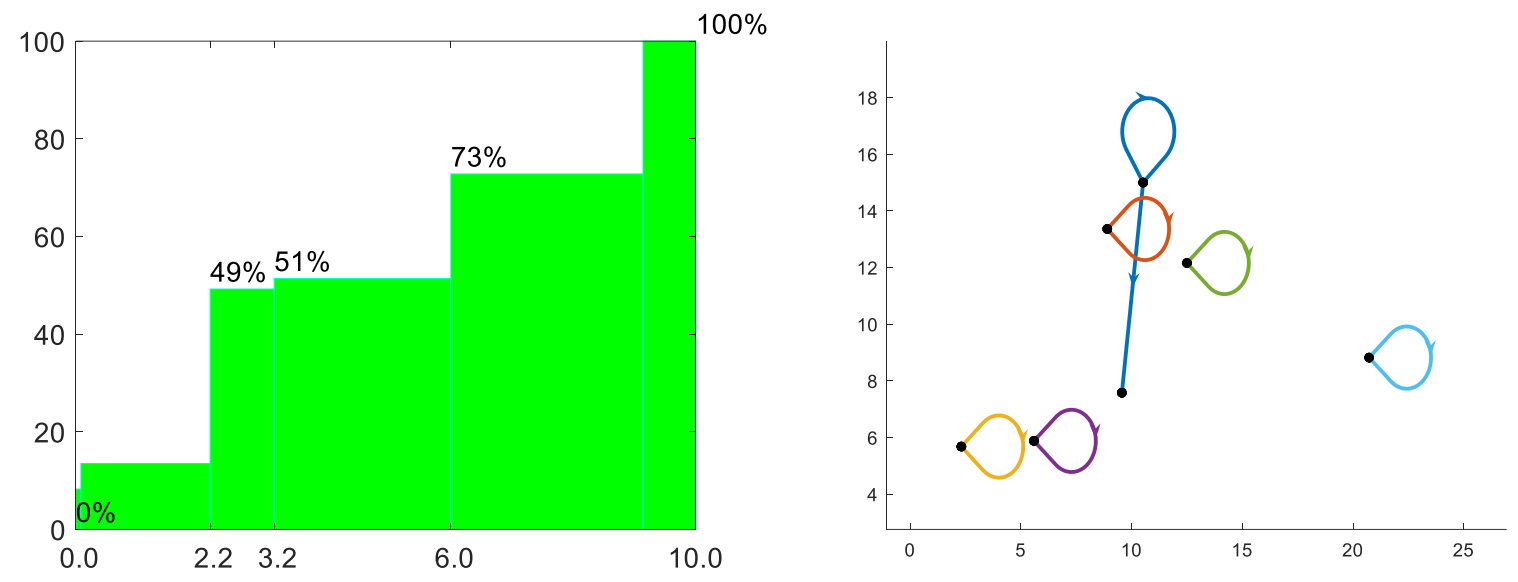

Figure 6-9. (a) Percentage of total restored load along time during the restoration

(b) Energization sequence for the electric power network
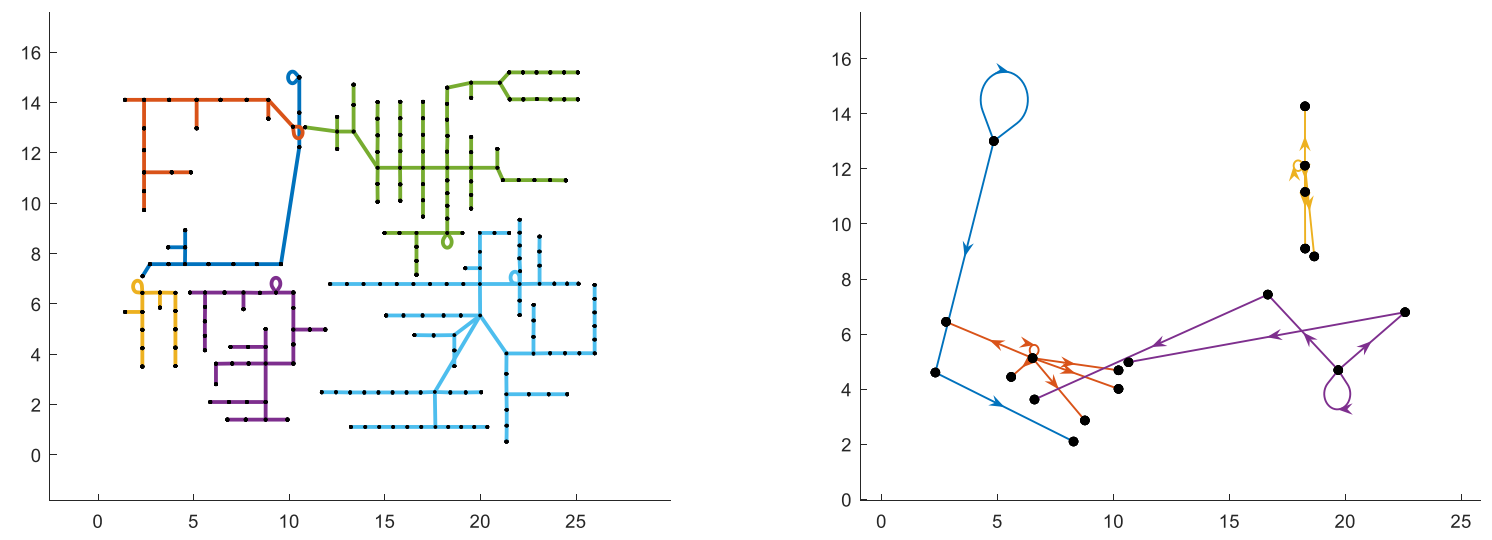

Figure 6-10 (a) Single-line diagram of energized system (b) Dispatch map for repair crews

\section{Significant Accomplishments and Conclusions:}

The significant accomplishments and outcomes obtained in this project are summarized as follows:

1. The developed framework enables a flexible operation paradigm toward improving the distribution grid resilience by fully leveraging the controllability, flexibility, and locational value of solar energy. In addition, the framework can provide customers with more interconnection choices and reduce PV curtailment during extreme conditions, and eventually enable solar energy to play a critical role in improving distribution grid resilience and further promote renewable energy deployment.

2. The resilience benefit of distributed PV and other DERs is investigated and justified through the developed framework on both a large-scale test system $(>10,000$ nodes) and a real feeder system. 
3. The pre-event preparation and post-event restoration and operation tasks contribute innovative methodologies to the state-of-art research community and demonstrate commercialization potential to be adopted by various stakeholders.

4. The developed methodologies have been published in multiple journal papers and presented in many conference panel sessions and IAB meetings. The promising results foster collaboration within the research and industry domains, including the Cooperative Research and Development Agreement (CRADA) signed with S\&C Electric Company.

The major technical challenges in this project are summarized as follows:

1. Coordinated preparation and operation for high PV penetration distribution grid. A key challenge is to coordinate pre-event preparation and post-event operation effectively while considering the uncertainties introduced by high penetration PV and other factors. The complexity of this project requires the problem to be formulated in a comprehensive manner and solved efficiently.

2. Requirement of 5-day operation. Existing methodologies using multi-time step formulation models need to generate solutions over a 5-day horizon will result in excessive time steps that are difficult to solve. How to effectively coordinate the PV and other DERs through the 5-day horizon is a challenge for the project team.

3. Scalability on large-size systems. Valuating the methodologies on large-scale systems poses a significant challenge for developing the solution algorithms.

\section{Inventions, Patents, Publications, and Other Results:}

\section{Peer-reviewed journal article}

1. Arif, Anmar, Zhaoyu Wang, Bo Chen, and Bo Chen. "Repair and resource scheduling in unbalanced distribution systems using neighborhood search." IEEE Transactions on Smart Grid 11, no. 1 (2020): 673-685.

2. Arif, Anmar, Zhaoyu Wang, Chen Chen, and Bo Chen. "A Stochastic MultiCommodity Logistic Model for Disaster Preparation in Distribution Systems." IEEE Transactions on Smart Grid 11, no. 1 (2019): 565-576.

3. Chen, Bo, Zhigang Ye, Chen Chen, and Jianhui Wang. "Toward a MILP modeling framework for distribution system restoration." IEEE Transactions on Power Systems 34, no. 3 (2018): 1749-1760.

4. Chen, Bo, Zhigang Ye, Chen Chen, Jianhui Wang, Tao Ding, and Zhaohong Bie. "Toward a synthetic model for distribution system restoration and crew dispatch." IEEE Transactions on Power Systems 34, no. 3 (2018): 2228-2239.

5. Jiayong Li, Chengying Liu, Mohammad E. Khodayar, etc., "Distributed online VAR control for unbalanced distribution networks with photovoltaic generation." IEEE Transactions on Smart Grid, vol. 11, no. 6 (2020): 4760-4772.

6. Jiayong Li, Mohammad E. Khodayar, Mohammad Ramin Feizi, "Hybrid Modeling Based Co-Optimization of Crew Dispatch and Distribution System Restoration Considering Multiple Uncertainties," IEEE Systems Journal, in press. 


\section{Conference publication}

1. Shanshan Ma, Nichelle'Le Carrington, Arif, Anmar, and Zhaoyu Wang. "Resilience assessment of self-healing distribution systems under extreme weather events." 2019 IEEE PES General Meeting, Atlanta, Aug. 2019. (Best Paper Award)

\section{Conference presentation}

1. Xiaonan Lu, Yoav Sharon, V. Mehr, Guohui Yuan, Shijia Zhao "Lessons Learned, Practice, and Methodologies for Renewables Integration and Grid Resilience using DERs and Networked Microgrids", panel session at ISGT-NA 2020, February, $19^{\text {th }}$, 2020

2. Xiaonan Lu, Ren Liu, Jianhui Wang, Shijia Zhao "Disaster Awareness, Restoration and Stability Enhancement of Resilient Distribution Grids with Increasing Penetration of Inverter-based Resources", panel session at ISGT-NA 2020, February, $18^{\text {th }}, 2020$

3. Bo Chen, Gary Oppedahl, Brian Patterson, Panel session at $5^{\text {th }}$ Grid Modernization Forum, May 20, 2020

4. Zhaoyu Wang, "Tutorial: power system resilience, fundamentals, analytical and planning tools, and industry practices", 2019 IEEE Innovative Smart Grid Technology Conference (ISGT 2019) (Washington DC), February 17, 2019.

5. Zhaoyu Wang, "Analyzing Utility Outage Data for Resilience Enhancement", 2019 IEEE Innovative Smart Grid Technology Conference (ISGT 2019) (Washington DC), Panel: Data Analytics for Grid Resilience Modeling and Enhancement Planning, February 18, 2019.

6. Zhaoyu Wang, "Data-driven Resilience Modeling and Enhancement", 2019 IEEE Innovative Smart Grid Technology Conference (ISGT 2019) (Washington DC), Panel: National Power Grid Resilience Modeling, February 20, 2019.

7. Zhaoyu Wang, "Data Analytics and Optimization for Enhancing Grid Resilience Against Extreme Weather Events", 2019 IEEE Power and Energy Society General Meeting (Atlanta, GA), Panel: Data-Driven Approaches for Mitigation of Natural Disasters Impacts on Power Grids, August 6, 2019.

\section{Path Forward:}

The methodologies developed in this project can be further extended to related research areas to address the existing and emerging challenges and bridge the gap with industrial adoption.

Resilience planning for renewable energies in distribution systems. Grid resilience can be further improved by strategically placing renewable energies to leverage their locational value and operational flexibility, as evidenced by the case studies in this project. In future work, a resilience-oriented planning framework can be developed to optimize the location, capacity, and functions of renewable energies, such that the renewable energies can be fully utilized for restoration during major power outages. 
Extended optimization framework considering smart inverter flexibility. As the smart inverter of PV and other DERs are supporting more functionalities (e.g., grid-forming control, grid-following control in $\mathrm{PQ}$ model and voltage regulation mode, configurable droop control), more resilience and operational flexibility benefits can be achieved by fully modeling and integrating these functionalities into the optimization framework.

Comprehensive risk-based optimization. When an extreme weather event hits an area, its corresponding distribution system may experience a failure-recovery-cost process. For example, the damaged transportation network hinders the physical delivery of flexible resources and decreases crew mobility. However, the trade-off between pre-event allocation cost and risk associated with damage loss under the upcoming event is not considered in the proposed pre-event preparation optimization model. The conditional value-at-risk $(\mathrm{CVaR})$ is a risk measure that focuses on high consequences and can be flexibly determined between the mean loss and the maximum loss. Therefore, in future work, the CVaR can be added in the pre-event preparation optimization model as a constraint to provide different risk preferences for the utility to make allocation decisions. Interdependency of critical infrastructures. It is critical to investigate the interdependencies of other critical infrastructures (e.g., communication, gas network, water) and their impact on power grid operation in face of natural disasters. An enhanced optimization framework should be developed to consider and even co-optimize these infrastructures.

The methodologies developed in the project can be adopted by multiple stakeholders. The project team will keep collaborating on further opportunities to partner with industrial entities to foster the technology transfer and commercialization adoption. The ANL team has developed a CRACA with S\&C Electric Company and will work together on protection and restoration-related topics and discuss the commercialization potential of the methodologies. The project team will keep in contact with the IAB members and local utility members to keep them aware of the future adoption of developed methodologies. The project team will also keep disseminating the project outcomes. 


\section{References:}

[E-1] Campbell, Richard J., and Sean Lowry. "Weather-related power outages and electric system resiliency." Washington, DC: Congressional Research Service, Library of Congress, 2012.

[E-2] "Fundamentals of Advanced Microgrid: ACER Course book, May 2019. [Online Available]:

https://www.usaid.gov/sites/default/files/documents/1865/ACER_Coursebook_2019_en glish.pdf

[B-1] A. Arif, Z. Wang, J. Wang, and C. Chen, "Power distribution system outage management with co-optimization of repairs, reconfiguration, and DG dispatch," IEEE Trans. Smart Grid, vol. 9, no. 5, pp. 4109-4118, Sept. 2018.

[B-2] A. Arif, S. Ma, Z. Wang, J. Wang, S. M. Ryan and C. Chen, "Optimization service restoration in distribution system with uncertain repair time and demand," IEEE Trans. Power Syst., vol. 33, no. 6, pp. 6828-6838, Nov. 2018.

[B-3] A. Arif, Z. Wang, C. Chen, and B. Chen, "A stochastic multi-commodity logistic model for disaster preparation in distribution systems," IEEE Trans. Smart Grid, vol. 11, no. 1, pp. 565-576, Jan. 2020.

[B-4] S. Ma, S. Li, Z. Wang, and F. Qiu, "Resilience-oriented distribution system design with decision-dependent uncertainty," IEEE Trans. Power Syst., vol. 34, no. 4, pp. 28802891, July 2019.

[B-5] S. Ma, L. Su, Z. Wang, F. Qiu and G. Guo, "Resilience enhancement of distribution grids against extreme weather events," IEEE Trans. Power Syst., vol. 33, no. 5, pp. 48424853, Sept. 2018.

[B-6] D. Lubkeman and D. E. Julian, "Large scale storm outage management," pp. 1622, 2005.

[B-7] C. T. Ingram, "Electric Utility Storm Restoration -Crew Work Allocation Optimization," PhD diss., Massachusetts Inst. Technol., pp. 1-56, 2007.

[B-8] A. Arab, A. Khodaei, Z. Han, and S. K. Khator, "Proactive Recovery of Electric Power Assets for Resiliency Enhancement," IEEE Access, 2015.

[B-9] P. Van Hentenryck and C. Coffrin, "Transmission system repair and restoration," Math. Program., 2015.

[B-10] C. Coffrin and P. Van Hentenryck, "Transmission system restoration with cooptimization of repairs, load pickups, and generation dispatch," Int. J. Electr. Power Energy Syst., 2015.

[B-11] P. Van Hentenryck, C. Coffrin, and R. Bent, "Vehicle routing for the last mile of power system restoration," in 17th Power Systems Computation Conference, PSCC 2011, 2011.

[B-12] M. E. Baran, and F. F. Wu, "Network reconfiguration in distribution systems for loss reduction and load balancing," IEEE Transactions on Power Delivery, vol. 4, no. 2, pp. 1401-1407, April 1989.

[B-13] P. Fortenbacher and T. Demiray, "Linear/quadratic programming-based optimal 
power flow using linear power flow and absolute loss approximations," International Journal of Electrical Power \& Energy Systems, vol. 107, pp. 680-689, 2019.

[B-14] T. Wang, M. Meskin, Y. Zhao, and I. Grinberg, "Optimal power flow in distribution networks with high penetration of photovoltaic units," in Electrical Power and Energy Conference (EPEC), 2017 IEEE. pp. 1-6.

[B-15] M. Javadi, T. Amraee, "Mixed integer linear formulation for undervoltage load shedding to provide voltage stability", Generation Transmission \& Distribution IET, vol. 12, no. 9, pp. 2095-2104, 2018.

[B-16] E. Hooshmand, and A. Rabiee, "Energy management in distribution systems, considering the impact of reconfiguration, RESs, ESSs and DR: A trade-off between cost and reliability," Renewable energy, 139, pp.346-358, 2019.

[B-17] M. B. Ndawula, P. Zhao, and I. Hernando-Gil, "Smart application of energy management systems for distribution network reliability enhancement," In Proc. 2018 IEEE International Conference on Environment and Electrical Engineering and 2018 IEEE Industrial and Commercial Power Systems Europe (EEEIC/I\&CPS Europe) pp. 1-5, June 2018.

[B-18] A. Azizivahed, A. Arefi, S. Ghavidel, M. Shafie-khah, L. Li, J. Zhang, and J. P. Catalão, "Energy management strategy in dynamic distribution network reconfiguration considering renewable energy resources and storage," IEEE Transactions on Sustainable Energy, vol. 11, no. 2, pp. 662-673, 2019.

[B-19] J. Paoli, B. Brinkmann, and M. Negnevitsky, "Optimising low-voltage transformer tap settings in distribution networks," in 2019 29th Australasian Universities Power Engineering Conference (AUPEC). IEEE, 2019, pp. 1-6.

[B-20] R. P. Xu, C. Zhang, Y. Xu, and Z. Y. Dong, "Rolling horizon based multi-objective robust voltage/var regulation with conservation voltage reduction in high pvpenetrated distribution networks," IET Generation, Transmission \& Distribution, vol. 13, no. 9, pp. 1621-1629, 2019.

[B-21] G. Brusco, A. Burgio, D. Menniti, A. Pinnarelli, and N. Sorrentino, "Energy management system for an energy district with demand response availability," IEEE Transactions on Smart Grid, vol. 5, no. 5, pp. 2385-2393, 2014.

[B-22] L. R. de Araujo, D. R. R. Penido, and F. de Alcântara Vieira, "A multiphase optimal power flow algorithm for unbalanced distribution systems," International Journal of Electrical Power \& Energy Systems, vol. 53, pp. 632-642, 2013.

[B-23] S. Bruno, S. Lamonaca, G. Rotondo, U. Stecchi, and M. La Scala, "Unbalanced three-phase optimal power flow for smart grids," IEEE Transactions on Industrial Electronics, vol. 58, no. 10, pp. 4504-4513, 2011.

[B-24] M. Farivar and S. H. Low, "Branch flow model: Relaxations and convexification part I," IEEE Transactions on Power Systems, vol. 28, no. 3, pp. 2554-2564, 2013.

[B-25] J. Lavaei and S. H. Low, "Zero duality gap in optimal power flow problem," IEEE Transactions on Power Systems, vol. 27, no. 1, p. 92, 2012.

[B-26] L. Gan, N. Li, U. Topcu, and S. H. Low, "Exact convex relaxation of optimal power 
flow in radial networks," IEEE Transactions on Automatic Control, vol. 60, no. 1, pp. 72-87, 2015.

[B-27] S. H. Low, "Convex relaxation of optimal power flow part I: Formulations and equivalence," IEEE Transactions on Control of Network Systems, vol. 1, no. 1, pp. 15-27, 2014.

[B-28] Low, Steven H. , "Convex relaxation of optimal power flow part II: Exactness," IEEE Transactions on Control of Network Systems, vol. 1, no. 2, pp. 177-189, 2014.

[B-29] E. Dall'Anese, H. Zhu, and G. B. Giannakis, "Distributed optimal power flow for smart microgrids." IEEE Trans. Smart Grid, vol. 4, no. 3, pp. 1464-1475, 2013.

[B-30] R. A. Jabr, "Radial distribution load flow using conic programming," IEEE transactions on power systems, vol. 21, no. 3, pp. 1458-1459, 2006.

[B-31] M. Jafari, Z. Malekjamshidi, D. D. C. Lu, and J. Zhu, "Development of a fuzzy-logicbased energy management system for a multiport multioperation mode residential smart microgrid," IEEE Transactions on Power Electronics, vol. 34, no.4, pp. 32833301, 2018.

[B-32] S. Leonori, M. Paschero, A. Rizzi, and F. M. F. Mascioli, "An optimized microgrid energy management system based on FIS-MO-GA paradigm," in Proc. IEEE Int. Conf. Fuzzy Syst. (FUZZ-IEEE), Naples, Jul. 2017, pp. 1-6.

[B-33] M. Jafari, Z. Malekjamshidi, J. Zhu, and M. H. Khooban, "A novel predictive fuzzy logic-based energy management system for grid-connected and off-grid operation of residential smart microgrids," IEEE Journal of Emerging and Selected Topics in Power Electronics, vol. 8, no.2, pp. 1391-1404, 2018.

[B-34] M. A. Hossain, H. R. Pota, S. Squartini, and A. F. Abdou, "Modified PSO algorithm for real-time energy management in grid-connected microgrids," Renewable energy, vol. 136, pp. 746-757, 2019.

[B-35] T. Lu, Z. Wang, Q. Ai, and W. J. Lee, "Interactive model for energy management of clustered microgrids. IEEE Transactions on Industry Applications," vol. 53, no. 3, pp. 1739-1750, 2017.

[B-36] T. Niknam, "A new approach based on ant colony optimization for daily Volt/Var control in distribution networks considering distributed generators," Energy Conversion and Management, vol. 49, no. 12, pp. 3417-3424, 2008.

[B-37] S. A. Arefifar, M. Ordonez, and Y. A.-R. I. Mohamed, "Energy management in multi-microgrid systems-development and assessment," IEEE Trans. Power Syst., vol. 32, no. 2, pp. 910-922, Mar. 2017.

[B-38] T. Morstyn, A. V. Savkin, B. Hredzak, and H. D. Tuan, "Scalable energy management for low voltage microgrids using multi-agent storage system aggregation," IEEE Trans. Power Syst., vol. 33, no. 2, pp. 1614-1623, Mar. 2018.

[B-39] M. W. Khan, J. Wang, M. Ma, L. Xiong, P. Li, and F. Wu, "Optimal energy management and control aspects of distributed microgrid using multi-agent systems," Sustainable Cities and Society, vol. 44, pp. 855-870, 2019. 
[B-40] Q. Peng and S. Low, "Distributed algorithm for optimal power flow on unbalanced multiphase distribution networks," arXiv preprint arXiv:1512.06482, 2015.

[B-41] B. Chen, et al. "Sequential service restoration for unbalanced distribution systems and microgrids," IEEE Transactions on Power Systems, vol. 33, no. 2, pp. 15071520, 2018.

[B-42] H. Wang, K. Meng, Z. Y. Dong, Z. Xu, F. Luo, and K. P. Wong, "Efficient real-time residential energy management through MILP based rolling horizon optimization,". In Proc. 2015 IEEE Power \& Energy Society General Meeting, pp. 1-6. 2015.

[B-43] R. Palma-Behnke, C. Benavides, F. Lanas, B. Severino, L. Reyes, J. Llanos, and D. Sáez, "A microgrid energy management system based on the rolling horizon strategy," IEEE Transactions on smart grid, vol. 4, no.2, pp. 996-1006, 2013.

[B-44] S. Ma, L. Su, Z. Wang, F. Qiu, and G. Guo, "Resilience enhancement of distribution grids against extreme weather events," IEEE Transactions on Power Systems, vol. 33, no. 5, pp. 4842-4853, 2018.

[B-45] X. Chen, W. Wu, and B. Zhang, "Robust restoration method for active distribution networks," IEEE Transactions on Power Systems, vol. 31, no. 5, pp. 4005-4015, 2016.

[B-46] A. R. Malekpour and A. Pahwa "Stochastic networked microgrid energy management with correlated wind generators" IEEE Transaction Power Systems, vol. 32 no. 5 pp. 3681-3693, 2017.

[B-47] "Battery Energy Storage Overview", [Online Available]: https://www.cooperative.com/programs-services/bts/documents/reports/batteryenergy-storage-overview-report-update-april-2019.pdf

[B-48] Y. Hou, R. Vidu, and P. Stroeve, "Solar Energy Storage Systems Review", [Online Available]: http://ucsolar.org/files/public/documents/Poster5.pdf

[B-49] "2018 Top Solar Storage Products", [Online Available]: https://www.solarpowerworldonline.com/2018-top-solar-storage-products/

[B-50] "2019-20 IRP: Proposed Reference System Plan", [Online Available]: https://www.cpuc.ca.gov/uploadedFiles/CPUCWebsite/Content/Utilities/ndustries/ Energy/EnergyPrograms/ElectPowerProcurementGeneration/irp/2018/2019\%20I RP\%20Proposed\%20Reference\%20System\%20Plan_20191106.pdf

[B-51] Vugrin, Eric, Anya Castillo, and Cesar Silva-Monroy. "Resilience metrics for the electric power system: A performance-based approach." Report: SAND2017-1493

[T2-1] B. Chen, C. Chen, J. Wang, and K. L. Butler-Purry, "Sequential service restoration for unbalanced distribution systems and microgrids," IEEE Trans. Power Syst., to be published.

[T2-2] A. Arif, Z. Wang, J. Wang, C. Chen. (May 7, 2019) "Repair and resource scheduling in unbalanced distribution systems using neighborhood search." Accessed: May 7, 2019. [Online]. Available: https://arxiv.org/abs/1808.10548

[T2-3] K. Zipp. "What are some common types of solar PV and storage installations?" Internet: https://www.solarpowerworldonline.com/2015/10/what-are-some- 
common-types-of-solar-pv-and-storage-installations/, Oct. 29, 2015 [Nov. 1, 2018].

[T2-4] C. Meehan. "What types of solar power systems can I get for my home?" Internet:https://www.solar-estimate.org/news/2017-11-15-types-solar-powersystems-homes-111517, Nov. 15, 2017 [Nov. 1, 2018].

[T2-5] T. Kenning. "Australia's first large-scale grid-connected solar and battery project comes online." Internet: https://www.pv-tech.org/news/australias-_rst-large-scalegrid-connected-solar-and-battery-project-comes, Feb. 19, 2018 [Nov. 1, 2018].

[T2-6] F. Y. Melhem, O. Grunder, Z. Hammoudan and N. Moubayed, "Energy management in electrical smart grid environment using robust optimization algorithm," IEEE Trans. Ind. Appl., vol. 54, no. 3, pp. 2714-2726, June 2018.

[T2-7] R. T. Rockafellar and R. J.-B. Wets, "Scenarios and policy aggregation in optimization under uncertainty," Math. Oper. Res., vol. 16, no. 1, pp. 119-147, 1991.

[T2-8] J.-P. Watson and D. L. Woodruff, "Progressive hedging innovations for a class of stochastic mixed-integer resource allocation problems," Comput. Manage. Sci., vol. 8, no. 4, pp. 355-370, Jul. 2010.

[T2-9] K. Cheung, D. Gade, S. Ryan, C. Silva-Monroy, J.-P. Watson, R. Wets, D. L. Woodruff, "Toward scalable stochastic unit commitment - part 2: assessing solver performance," Energy Syst., vol. 6, pp. 417-438, Apr. 2015.

[T2-10] IEEE PES AMPS DSAS Test Feeder Working Group, "123-bus feeder." Internet: http://sites.ieee.org/pes-testfeeders/resources/, Feb. 3, 2014 [May 12, 2018].

[T2-11] W. E. Hart, C. Laird, J. P. Watson, and D. L. Woodruff, Pyomo - optimization modeling in Python (Springer optimization and its applications). New York, NY, USA, Springer, 2012, vol. 67.

[T3-1] X. Chen, W. Wu, and B. Zhang, "Robust restoration method for active distribution networks," IEEE Transactions on Power Systems, vol. 31, no.5, pp. 4005-4015, 2016.

[T3-2] B. Chen, et al. "Sequential service restoration for unbalanced distribution systems and microgrids," IEEE Transactions on Power Systems, vol. 33, no. 2, pp. 15071520, 2018.

[T4-1]S. Ma, B. Chen, and Z. Wang, "Resilience Enhancement Strategy for Distribution Systems under Extreme Weather Events," IEEE Trans. Smart Grid, vol. 9, no. 2, pp. 1442-1451, Mar. 2018.

[T4-2]R. E. Brown, "Cost-benefit analysis of the deployment of utility infrastructure upgrades and storm hardening programs," Quanta Technol. Raleigh, 2009.

[T4-3] “Forward Speed of a Hurricane," Hurricane Research Division. [Online]. Available: https://www.aoml.noaa.gov/hrd/tcfaq/G16.html.

[T4-4]P. Javanbakht and S. Mohagheghi, "A risk-averse security-constrained optimal power flow for a power grid subject to hurricanes," Electr. Power Syst. Res., vol. 116, pp. 408-418, Nov. 2014. 
[T4-5]N. Lugeri, Z. W. Kundzewicz, E. Genovese, S. Hochrainer, and M. Radziejewski, "River flood risk and adaptation in Europe-assessment of the present status," Mitig. Adapt. Strateg. Glob. Change, vol. 15, no. 7, pp. 621-639, Oct. 2010.

[T4-6]S. D. Seyoum, Z. Vojinovic, R. K. Price, and S. Weesakul, "Coupled 1D and Noninertia 2D Flood Inundation Model for Simulation of Urban Flooding," J. Hydraul. Eng., vol. 138, no. 1, pp. 23-34, Jan. 2012.

[T4-7]J. Leandro, A. S. Chen, S. Djordjević, and D. A. Savić, "Comparison of 1D/1D and 1D/2D Coupled (Sewer/Surface) Hydraulic Models for Urban Flood Simulation," J. Hydraul. Eng., vol. 135, no. 6, pp. 495-504, Jun. 2009.

[T4-8]J. S. O'Brien, P. Y. Julien, and W. T. Fullerton, "Two-Dimensional Water Flood and Mudflow Simulation,” J. Hydraul. Eng., vol. 119, no. 2, pp. 244-261, Feb. 1993.

[T4-9]L. E. Kollar and M. Farzaneh, "Vibration of Bundled Conductors Following Ice Shedding," IEEE Trans. Power Deliv., vol. 23, no. 2, pp. 1097-1104, Apr. 2008.

[T4-10]B. E. K. Nygaard, I. A. Seierstad, and A. T. Veal, "A new snow and ice load map for mechanical design of power lines in Great Britain," Cold Reg. Sci. Technol., vol. 108, pp. 28-35, Dec. 2014.

[T4-11]"Atmospheric icing of stuctures," Electric Power Research Institutue.

[T4-12]P. Fu, M. Farzaneh, and G. Bouchard, "Two-dimensional modelling of the ice accretion process on transmission line wires and conductors," Cold Reg. Sci. Technol., vol. 46, no. 2, pp. 132-146, Nov. 2006.

[T4-13]C. Ji, Y. Wei, and H. V. Poor, "Resilience of Energy Infrastructure and Services: Modeling, Data Analytics, and Metrics," Proc. IEEE, vol. 105, no. 7, pp. 13541366, Jul. 2017.

[T4-14]Bjarnadottir Sigridur, Li Yue, and Stewart Mark G., "Hurricane Risk Assessment of Power Distribution Poles Considering Impacts of a Changing Climate," J. Infrastruct. Syst., vol. 19, no. 1, pp. 12-24, Mar. 2013.

[T4-15]A. Arab, A. Khodaei, S. K. Khator, K. Ding, V. A. Emesih, and Z. Han, "Stochastic Pre-hurricane Restoration Planning for Electric Power Systems Infrastructure," IEEE Trans. Smart Grid, vol. 6, no. 2, pp. 1046-1054, Mar. 2015.

[T4-16]“HAZUS Flood Model Technical Manual," Federal Emergency Management Agency.

[T4-17]Y. Li and B. R. Ellingwood, "Hurricane damage to residential construction in the US: Importance of uncertainty modeling in risk assessment," Eng. Struct., vol. 28, no. 7, pp. 1009-1018, Jun. 2006.

[T4-18]“Lesson: Pole Structural Loading," Red Vector.

[T4-19]E. Broström and L. Söder, "Ice Storm Impact on Power System Reliability," presented at the 12 th International Workshop on Atmospheric Icing on Structures (IWAIS 2007), Yokohama, Japan, 2007.

[T4-20] lowa Distribution Test Systems, http://wzy.ece.iastate.edu/Testsystem.html. 



\section{Argonne}

\section{Energy Systems Division}

Argonne National Laboratory

9700 South Cass Avenue, Bldg. 362

Lemont, IL 60439-4832

www.anl.gov 